Chapter 1 in Advances in Chemical Engineering vol. 48 - Photobioreaction Engineering, editor J. Legrand, pp 1-107. Academic Press, UK, 2016

\title{
Photobioreactor modeling and radiative transfer analysis for engineering purposes
}

\author{
Jérémi Dauchet ${ }^{\mathrm{a}, \mathrm{b}, *}$, Jean-François Cornet $^{\mathrm{a}, \mathrm{b}},{\text { Fabrice } \text { Gros }^{\mathrm{a}, \mathrm{b}} \text {, Matthieu Roudet }}^{\mathrm{a}, \mathrm{b}}$, C.-Gilles Dussap ${ }^{\mathrm{b}, \mathrm{c}}$ \\ ${ }^{a}$ Université Clermont Auvergne, ENSCCF, BP 10448, F-63000 Clermont-Ferrand, France \\ ${ }^{b}$ CNRS, UMR 6602, Institut Pascal, F-63178 Aubière, France \\ ${ }^{c}$ Université Clermont Auvergne, Université Blaise Pascal, BP 10448, F-63000 Clermont-Ferrand, France
}

\begin{abstract}
The present chapter introduces the theoretical framework for constructing predictive knowledge-models leading to the calculation of the volumetric rate of biomass production, the surface rate of biomass production and the thermodynamic efficiency of photobioreactors. Here, the main assumption is that photosynthesis reaction is limited by radiative transfer only. First, the predictive determination of the scattering and absorption properties of photosynthetic microorganisms of various types is addressed. Then, these radiative properties are used to calculate the radiation field within the reaction volume by solving the radiative transfer equation. Both the development of approximate solutions appropriated with typical photobioreactor configurations (intermediate scattering optical-thickness) and the rigorous solution of the radiative transfer equation by the Monte Carlo method are addressed, including the treatment of complex geometric structures. Finally, the thermokinetic coupling between the radiation field, the photosynthesis reaction rates and thermodynamic efficiency are investigated. For the special case of the cyanobacterium Arthrospira platensis, a complete stoichiometric, kinetic and thermodynamic model is constructed using the linear thermodynamics of irreversible processes to analyze the primary events of photosynthesis (Z-scheme). Comparison between the theoretical calculations presented in this chapter and experimental results confirms the ability of the proposed predictive approach, after parameters reification, to quantify performances of many kinds of photobioreactors (geometry, size) functioning under different operating conditions. An extension of the proposed coupling approach for the more complicated case of eukaryotic (microalgae) micro-organisms is then proposed as further perspective of this work.
\end{abstract}

Keywords: photobioreactor, predictive model, optical and radiative properties, light transfer, Monte Carlo method, thermokinetic coupling, photosynthesis, thermodynamic efficiency, cyanobacteria, microalgae

\section{Introduction}

During the past decades, photobioreactors have found promising applications, in particular for highvalue products, for example, in pharmacy, cosmetics, and aquaculture feeds. Nevertheless, the development of industrial photobioreactors still requires optimization efforts, especially for achieving a high volumetric production rate in the context of large-scale implementation. The necessary technological breakthroughs are even more radical for application to energy carrier production for development of algal biorefineries. Here, the main criterion for viability of solar-energy processes is their thermodynamic efficiency. Although it is

\footnotetext{
* Corresponding author

Email address: jeremi.dauchet@univ-bpclermont.fr (Jérémi Dauchet)
} 
nowadays believed that such processes should operate at $\sim 10 \%$ thermodynamic efficiency to be competitive with other solar-energy technologies, most photobioreactor concepts that are reported in the literature, including state-of-the-art developments, operate between 0.2 and $3 \%$. Photosynthesis of a single microalga is capable of such efficient $(>10 \%)$ energy conversion [1], but here, the obstacle is implementation at the photobioreactor scale, which still requires significant advances in chemical engineering.

We believe that these technological improvements (the optimization of the volumetric production rate and thermodynamic efficiency) can be achieved only by constructing predictive models compatible with the requirements of photobioreactor simulation, design, sizing, scale-up, optimization, and model-based predictive control. On the one hand, such models must be generic to be suitable for testing of a wide range of technological strategies and operating modes; this requirement entails strong theoretical bases and advanced experimental studies. On the other hand, these models must be numerically tractable and compatible with analysis of the process; this requirement means identification of appropriate approximations and numerical methods. The present chapter is an overview of our practice of photobioreactor modeling and is aimed at fulfilling the above needs. The resulting models are validated at the end of this chapter (in Section 5.6) and used in a separate chapter of this book (Chapter 5 by Pruvost et al.) for analysis of the process.

Photobioreactor engineering addresses optimization of the volumetric rate of biomass production, the surface rate of biomass production (with respect to the solar-energy collecting surface), and thermodynamic efficiency of the process as well as biomass composition (i.e., the biomass quality). Hereafter, we mainly focus on construction of a predictive model for the volumetric rate $\left\langle r_{x}\right\rangle$ (e.g., expressed in $k g$, or moles of dry biomass per second and per $m^{3}$ of the reaction volume). This is the main difficulty with assessing performance of a photobioreactor because most of the other parameters of interest can be deduced only from the value of $\left\langle r_{x}\right\rangle$, in a rather straightforward manner (see Section 5).

Our model is based on integral formulation of the photobioreactor's volumetric rate; this approach is extremely convenient for analyzing the interaction between the mechanisms involved at different scales of the process:

$$
<r_{x}>=\frac{1}{V} \int_{\mathcal{V}} r_{x}(\mathbf{x}) d \mathbf{x}
$$

where $\left\langle r_{x}\right\rangle$ is the average local volumetric rate $r_{x}(\mathbf{x})$ at location $\mathbf{x}$, calculated across the geometric domain $\mathcal{V}$ of microorganism culture with volume $V$. In the text that follows, we focus on perfectly stirred photobioreactors where the microorganism concentration $C_{x}$ (i.e., the dry-biomass concentration) is uniform within $\mathcal{V}$. This assumption may be easily extended to plug-flow photobioreactors, in which intensive variables (such as $C_{x}$ ) are homogeneous within the surface perpendicular to the flow. In this case, $\left\langle r_{x}\right\rangle$ is obtained as in Eq. 1 from a surface integral, and $\left\langle r_{x}(z)\right\rangle$ is used in the differential mass balance of the photobioreactor [2]. In these situations, the local volumetric rate is

$$
r_{x}(\mathbf{x})=C_{x} J_{x}(\mathbf{x})
$$

where $J_{x}(\mathbf{x})$ is the average rate of biomass production by a photosynthetic microorganism within the infinitesimal volume element $d \mathbf{x}$ around the location $\mathbf{x}$ (usually expressed in moles per second per $\mathrm{kg}$ of dry biomass $^{1}$ ). In Eqs. 1 and $2,\left\langle r_{x}\right\rangle$ is the production rate at the scale of the process, $r_{x}$ is the local rate within the reaction volume, and $J_{x}$ is the production rate of an isolated photosynthetic cell. With this approach, prediction of $J_{x}$ is the central question.

The construction of a model for the specific rate of biomass production $J_{x}$ is presented in Section 5 . The main assumption of this model is that the photosynthetic reaction is limited by radiative transfer only. Indeed, ensuring proper mixing and maintenance of all physiological needs under their optimal conditions ( $\mathrm{pH}$, temperature, dissolved $\mathrm{CO}_{2}$, and minerals) are quite straightforward at the current state of knowledge in chemical engineering. Under these conditions, it has been clearly demonstrated in the past decades that photobioreactors are mainly governed by light transfer inside the culture volume; this transfer determines the

\footnotetext{
${ }^{1}$ The specific rate of biomass production by a microorganism is $J_{x}(\mathbf{x})$, expressed in moles per second per $k g$ of dry biomass, multiplied by the average dry mass of one microbial cell.
} 
kinetic rates, thermodynamic efficiency, biomass composition, and pigment content $[2,3,4,5,6,7,8,9,10]$. Hereafter, we focus on photobioreactors operating under such optimal conditions; therefore, we assume that $J_{x}$ is a function of radiative quantities only.

The early-primary events during photosynthesis (in photosystems) involve absorption of light within the spectral range of photosynthetically active radiation (PAR); this absorption generates electronic excitation events in the light-harvesting protein and causes water splitting by the oxygen-evolving complex. The characteristic time scale for this complete mechanism (consisting of a five-step cycle) is $\sim 1 \mathrm{~ms}$; therefore, we assume that the above processes are a function of local light absorption (cell displacement due to mixing is negligible within this time frame). Moreover, we assume that the contributions of electronic excitation generated by any photon within PAR are identical, regardless of the frequency of the photons ${ }^{2}$. Therefore, our model for photosystems is formulated as a function of the specific rate of photon absorption $\mathcal{A}(\mathbf{x})$, that is, the number of photons within PAR that are absorbed per unit of time and per $k g$ of dry biomass $(i . e .$, by the microorganism) at location $\mathbf{x}$. The following mechanisms underlie production of $A T P$ and $N A D P H_{2}$ within the photosynthetic electron transport chain, or the Z-scheme. In Section 5, we propose to use thermodynamics of irreversible processes [12], which is appropriate for modeling electron transport chains in a predictive manner, with a limited number of free parameters $[13,14]$. In the present case, the validity conditions of the approach require addressing physical quantities averaged across a few minutes [13, 14]; this period is long compared to the typical mixing time within photobioreactors (i.e., the time necessary for a microorganism to "explore" the reaction volume $\mathcal{V}$ ). Therefore, we will construct a model for the Z-scheme that is formulated as a function of averages $\langle f(\mathcal{A})\rangle$ across the local field of the absorption rate $\mathcal{A}$ :

$$
<f(\mathcal{A})>=\frac{1}{V} \int_{\mathcal{V}} f(\mathcal{A}(\mathbf{x})) d \mathbf{x}
$$

where $f$ is a function that is determined by an optimization procedure (see Section 5). The remaining mechanisms correspond to the synthesis of complex organic molecules (the biomass) via metabolic reactions called the dark reactions because they are independent of radiative transfer (they are driven only by the $A T P$ and $N A D P H_{2}$ generated by the light reactions discussed above). Altogether, our model for the specific rate of biomass production $J_{x}$ is a function of the specific rate of photon absorption $\mathcal{A}$ and averages $<f(\mathcal{A})>$ calculated across the reaction volume:

$$
J_{x}(\mathbf{x}) \equiv J_{x}(\mathcal{A}(\mathbf{x}),<f(\mathcal{A})>)
$$

where $J_{x}$ depends on the location $\mathbf{x}$ only via the absorption rate $\mathcal{A}(\mathbf{x})$.

The purpose of a photobioreactor is to absorb incident light in order to convert it into biomass via coupling with photosynthesis. On the one hand, efficient light absorption usually corresponds to heterogeneous radiation fields $\mathcal{A}(\mathbf{x})$ within the reaction volume (see Section 3). On the other hand, the coupling law (Eq. 4) is usually a non-linear function of $\mathcal{A}(\mathbf{x})$ (the law obtained in Section 5 is non-linear, but this is also the case for most of other models reported in the literature). Therefore, the coupling between radiative transfer and photosynthesis must be formulated locally ${ }^{3}$, which implies that determination of the volumetric rate $\left\langle r_{x}>\right.$ requires

1. estimating the radiation field $\mathcal{A}(\mathbf{x})$ within the culture volume (and averages $<f(\mathcal{A})>$ ),

\footnotetext{
${ }^{2}$ This assumption is always true for polychromatic-illumination and for monochromatic-illumination experiments in the case of eukaryotic microalgae, according to the well-known action spectrum of photosynthesis. This is not the case for monochromatic illumination of cyanobacteria [11]

${ }^{3}$ If the coupling law is a linear function of $\mathcal{A}(\mathbf{x})$, for example $J_{x}(\mathbf{x})=a \mathcal{A}(\mathbf{x})+b$, where $a$ and $b$ are constants, then determination of $r_{x}$ requires only the knowledge of $\langle\mathcal{A}\rangle$ (see Eq. 6): the coupling does not have to be formulated locally. Indeed, after substitution of the above-mentioned linear expression for $J_{x}$ into Eq. 2, Eq. 1 leads to

$$
<r_{x}>=C_{x} \frac{1}{V} \int_{\mathcal{V}}(a \mathcal{A}(\mathbf{x})+b) d \mathbf{x}=C_{x}(a<\mathcal{A}>+b)
$$
}


2. estimating the field of the specific rate of biomass production $J_{x}(\mathbf{x})$ according to Eq. 4 ,

3. estimating the field of the local rate $r_{x}(\mathbf{x})$ according to Eq. 2,

4. solving the integral across the culture volume in Eq. 1.

Then, the surface rate of biomass production $\left\langle s_{x}>\right.$ is obtained as

$$
<r_{x}>=a_{\text {light }}<s_{x}>
$$

where $a_{\text {light }}$ is the specific illuminated surface $a_{\text {light }}=S_{\text {light }} / V, S_{\text {light }}$ is the area of the illuminated surface (e.g., the solar-energy collecting surface), and $V$ is the reaction volume (including dark zones). Therefore, the surface rate is calculated from the volumetric rate and a purely geometric characteristic of the process. Finally, the thermodynamic efficiency $\left\langle\eta_{t h}>\right.$ of photosynthesis within the reaction volume is obtained from the value of $\left\langle r_{x}\right\rangle$ and from the average rate of photon absorption $\langle\mathcal{A}\rangle$ (see Section 5 ), where

$$
<\mathcal{A}>=\frac{1}{V} \int_{\mathcal{V}} \mathcal{A}(\mathbf{x}) d \mathbf{x}
$$

Thus, the construction of predictive models of photobioreactors requires careful formulation of radiative transfer within the reaction volume, in order to obtain the radiation field ( $c f$. step 1 in the above procedure). Such analysis is developed in the present chapter, starting in Section 2 with determination of the light scattering and absorption properties of photosynthetic-microorganism suspensions. Next, these properties are used in Section 3 for analysis of radiative transfer and in Section 4 for rigorous solution of the radiative transfer equation by the Monte Carlo method. Finally, the thermokinetic coupling between radiative transfer and photosynthesis is addressed in Section 5. It should be noted that Sections 2 and 4 mainly summarize works that have been already published elsewhere, whereas Sections 3 and 5 include extensive original work and results.

The main steps in our model and their organization within this chapter are summarized in Fig. 1. Our practice of the Monte Carlo method extends beyond the solution of the radiative transfer equation: in Section 4, we also argue that the Monte Carlo method is well suited for numerical implementation of the entire model, especially in research on photobioreactors with complex geometric structure. 


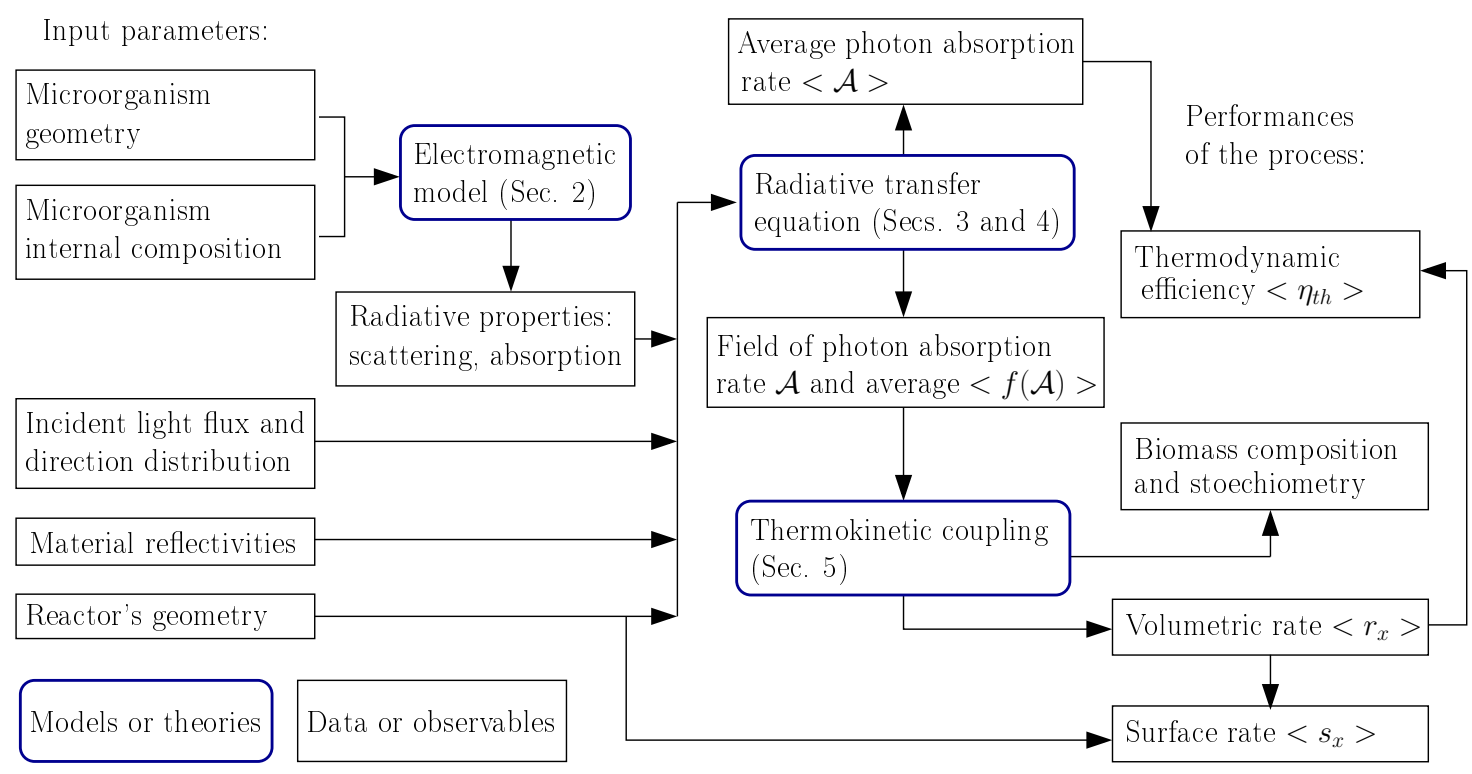

Figure 1: An outline of the predictive model presented in this chapter. Numerical implementation of the entire model by the Monte Carlo method is discussed in Section 4. 


\section{Calculating the radiative properties of photosynthetic microorganisms}

Any radiative analysis of photobioreactors starts with determination of absorption and scattering properties of the photosynthetic microorganism under study. This question is not trivial, and to the best of our knowledge, no available database provides adequate spectral and angular information that is needed, even for the strains of microalgae that are currently widely cultivated. Determination of these properties involves either highly specialized experiments $[9,15,16,17,18,19,20,21,22]$ (see also Chapter 2 by L. Pilon within the present book) or construction of a model implying solution of Maxwell's equations for particles with the types of heterogeneity, sizes, and shapes for which the usual numerical methods such as Lorenz-Mie, T-Matrix, finite-difference time-domain (FDTD), and discrete dipole approximation (DDA) [23, 24] are still impractical in many cases. The present section addresses the electromagnetic modeling approach, and later, we present the main steps of a methodological chain detailed in [25] for predictive calculation of the radiative properties within PAR. This methodological chain is derived from the expertise of the oceanographic community, and more broadly, of all the research fields that deal with the wave-particle interaction problem in the areas of atmospheric sciences, astrophysics, or engineering. In this broad context, our concern is to take into account not only the specificity of photosynthetic microorganisms but also analysis and optimization requirements of photobioreactor engineering. Therefore, this predictive method is based on limited and "easily" accessible experimental parameters (morphological and structural characteristics as well as photosynthetic-pigment content), allowing us to account for microorganisms' variability from one species to another and as a function of culture conditions (in particular, the illumination conditions) [11, 26, 27]. In Section 2.1 the basic concepts for the construction of such a predictive method are introduced and the choices made in [25] are presented: we construct an electromagnetic model of the light-particle interaction that is consistent with available protocols for determination of input parameters. The results produced by this methodological chain in the case of Chlamydomonas reinhardtii and Rhodospirillum rubrum under standard subculture conditions are discussed in Section 2.2. Finally, some perspectives for further development of the approach are drawn in Section 2.3.

\subsection{The methodological chain}

The radiative properties are input parameters of the radiative transfer equation (see Section 3): the absorption coefficient $k_{a, \nu}$, the scattering coefficient $k_{s, \nu}$, the extinction coefficient $k_{e x t, \nu}=k_{a, \nu}+k_{s, \nu}$ and the single-scattering phase function $p_{\boldsymbol{\Omega}, \nu}\left(\boldsymbol{\omega} \mid \boldsymbol{\omega}^{\prime}\right)$. The coefficients $k_{a, \nu}, k_{s, \nu}$, and $k_{e x t, \nu}\left(\right.$ expressed in $\left.m^{-1}\right)$ characterize attenuation of radiative intensity during passage through a microorganism suspension because of absorption, scattering, and extinction, respectively: they are coefficients from Bouguer's exponential law of attenuation (sometimes called Beer's law). The phase function $p_{\boldsymbol{\Omega}, \nu}\left(\boldsymbol{\omega} \mid \boldsymbol{\omega}^{\prime}\right)$ is the distribution of scattering directions $\boldsymbol{\omega}$ when radiation with incident direction $\boldsymbol{\omega}^{\prime}$ is scattered. These properties are a function of the frequency $\nu$ of radiation; under the assumption of perfect mixing (microorganism locations are statistically distributed uniformly), they are homogeneous within the reaction volume.

Our model implies independent scattering (the assumption that we share with the great majority of photobioreactor researchers). Indeed, typical biomass concentrations within the process are low enough to reasonably assume that each microbial cell interacts with radiation independently. We can therefore define particle cross-sections $\sigma$ that characterize the radiative properties of microbial cells independently of their concentration $C_{x}: k_{a, \nu}=C_{x} \sigma_{a, \nu}, k_{s, \nu}=C_{x} \sigma_{s, \nu}$, and $k_{e x t, \nu}=C_{x} \sigma_{e x t, \nu}$, where $\sigma_{e x t, \nu}=\sigma_{a, \nu}+\sigma_{s, \nu}$. To be precise, the cross-sections and the phase function characterize the interaction between an incident electromagnetic plane wave with frequency $\nu$ and a particle with given geometric structure and internal refractive index (as shown in Fig. 2). By solving this electromagnetic problem (i.e., by solving Maxwell's equations), one can calculate the radiative properties $\hat{\sigma}_{a, \nu}, \hat{\sigma}_{s, \nu}, \hat{\sigma}_{e x t, \nu}$, and $\hat{p}_{\boldsymbol{\Omega}, \nu}$ of an isolated particle with a specific shape, size, orientation, and refractive index. Then, under the assumption of independent scattering, the radiative properties of a perfectly stirred suspension are the average properties of isolated particles within the suspension. From now on, we will assume that every microbial cell within the photobioreactor has the same shape and refractive index; we use only the average value across orientation and size distributions:

$$
\sigma_{\nu}=\int_{\mathcal{D}_{\mathbf{E}_{o}}} d \mathbf{e}_{o} p_{\mathbf{E}_{o}}\left(\mathbf{e}_{o}\right) \int_{0}^{\infty} d r_{e q} p_{R_{e q}}\left(r_{e q}\right) \hat{\sigma}_{\nu}\left(\mathbf{e}_{o}, r_{e q}\right)
$$




$$
p_{\boldsymbol{\Omega}, \nu}\left(\boldsymbol{\omega} \mid \boldsymbol{\omega}^{\prime}\right)=\int_{\mathcal{D}_{\mathbf{E}_{o}}} d \mathbf{e}_{o} p_{\mathbf{E}_{o}}\left(\mathbf{e}_{o}\right) \int_{0}^{\infty} d r_{e q} p_{R_{e q}}\left(r_{e q}\right) \frac{\hat{\sigma}_{s, \nu}\left(\mathbf{e}_{o}, r_{e q}\right) \hat{p}_{\boldsymbol{\Omega}, \nu}\left(\boldsymbol{\omega} \mid \boldsymbol{\omega}^{\prime} ; \mathbf{e}_{o}, r_{e q}\right)}{\sigma_{s, \nu}}
$$

where

- Eq. 7 is valid for the three cross-sections $\sigma_{a, \nu}, \sigma_{s, \nu}$, and $\sigma_{e x t, \nu}$,

- $\mathbf{e}_{o}$ is a vector defining orientation of the particle (see Fig. 2), $p_{\mathbf{E}_{\mathbf{o}}}\left(\mathbf{e}_{\mathbf{o}}\right)$ is the orientation distribution, and $\mathcal{D}_{\mathbf{E}_{o}}$ is the domain of all possible orientations (for axisymmetric particles, $\mathcal{D}_{\mathbf{E}_{o}}$ is the total solid angle),

- $r_{e q}$ is the radius of the volume-equivalent sphere (that characterizes the size of the particle), and $p_{R_{e q}}\left(r_{e q}\right)$ is its distribution (i.e., the size distribution),

- the radiative properties $\hat{\sigma}_{\nu}$ and $\hat{p}_{\boldsymbol{\Omega}, \nu}$ of an isolated particle are a function of its orientation $\mathbf{e}_{o}$, size $r_{e q}$, shape, and internal refractive index $m_{\nu}$ as well as the frequency $\nu$ of incident radiation and the refractive index of the surrounding medium. Here, the surrounding medium is assumed to be non-absorbing, with the real refractive index $n_{e, \nu}$ equal to that of water [28].

Actually, the scattering problem in Fig. 2 is not affected by $m_{\nu}$ and $n_{e, \nu}$ but is influenced by the relative refractive index $m_{r, \nu}=m_{\nu} / n_{e, \nu}$. Similarly, the scattering problem is not affected by $r_{e q}$ and $\nu$ but is influenced by the ratio size/wavelength. This value is usually characterized by the size parameter $x=\frac{2 \pi r_{e q}}{\lambda}$, where $\lambda=c / \nu$ is the wavelength in the surrounding medium (water).

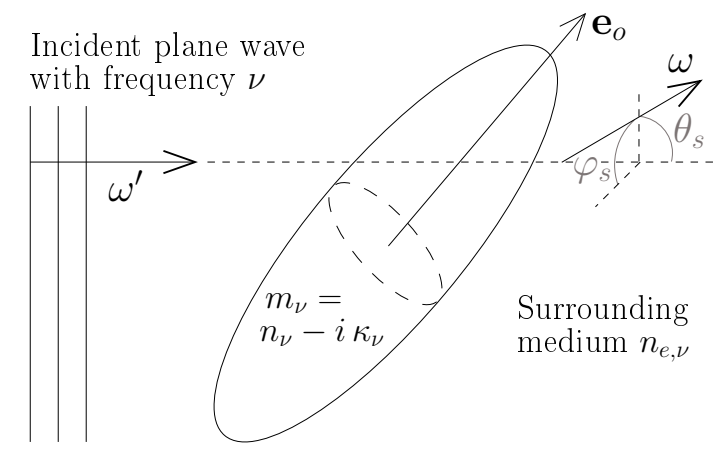

Figure 2: The scattering problem illustrated for a spheroidal particle with orientation $\mathbf{e}_{o}$ and effective refractive index $m_{\nu}=$ $n_{\nu}-i \kappa_{\nu}$. The surrounding medium is non-absorbing, with the real refractive index $n_{e, \nu}$. The speed of light within the medium is $c=c_{0} / n_{e, \nu}$, where $c_{0}$ is the speed of light in vacuum. The incident plane wave has frequency $\nu(i . e .$, wavelength $\lambda=c / \nu)$ and a wave vector collinear to $\omega^{\prime}$. A propagation direction of the radiation scattered by the particle is denoted as $\omega$. $\theta_{s}$ is the angle between $\omega$ and $\omega^{\prime}$.

Therefore, determination of the radiative properties implies construction of a model of the microorganism itself (its geometric structure and its internal features in terms of the refractive index) as well as implementation of the standard model of electromagnetism (solution of Maxwell's equations). These two tasks are actually interlocked because according to the literature [23, 24, 29], internal heterogeneity and shape of the most typical microorganisms correspond to ineluctable numerical difficulties with solution of Maxwell's equations. Consequently, we constructed an approximate electromagnetic model that involves simplification of the particles' description, with the corresponding approximations being chosen in line with the requirements of a photobioreactor study. The first choice is the following: we refuse to accept a compromise on the information about shape because we believe that this characteristic is essential for the interaction with radiation and is the key factor that distinguishes different species of microorganisms. On the other hand, we are willing to define the internal heterogeneity as an approximation of an effective homogeneous medium. Indeed, this approximation has been tested in many situations and appears to distort only the power scattered at large angles $[30,31,32,33]$. We selected this assumption because a very small proportion 
of the incident power is backscattered by a microorganism (90\% of the scattered power is usually confined to a solid angle of roughly $20^{\circ}$ aperture around the incident direction, see Section 2.2). As for the targeted radiative configurations, backscattering has a limited influence: the few backscattered photons have a very limited impact on radiative transfer within photobioreactors. Therefore, in order to alleviate the numerical difficulties, we modified our description of the scatterers, making them pseudo-homogeneous particles.

Various methods are available for solving the problem of an electromagnetic wave scattered by a homogeneous particle. Each of these methods is limited by the range of geometric structures and refractive indices that can be tackled $[23,24,34,35,36,29]$. Identifying those that are appropriate for the studies of photosynthetic microorganisms (from the pragmatic point of view) involves research on the available formal solutions (including approximations) and numerical approaches enabling their implementation, considering that

1. the size parameter $x=\frac{2 \pi r_{e q}}{\lambda}$ of microbial cells cultivated in a photobioreactor ranges from 5 to 200 ,

2. many microorganisms are strongly elongated particles that can be up to 50 times longer than their width, implying a very small radius of curvature,

3. microorganisms have the relative refractive index $m_{r, \nu}=n_{r, \nu}-i \kappa_{r, \nu}$ corresponding to low dielectric contrast with the surrounding medium $\left(n_{r, \nu} \in[1.02,1.2], \kappa_{r, \nu} \in\left[10^{-5}, 10^{-2}\right]\right)$.

The above description corresponds to a defined situation where very little can be done at present (see [37] regarding mineral dust, [38] regarding ice crystals, and [36] for an overview). Computation of the rigorous solution of Maxwell's equations is indeed usually achieved by numerical methods that, to our knowledge, fail to address the combination of the first two criteria listed above [23, 24, 36, 29]. The ongoing development of these methods (e.g., $[23,39,40,41,42,43])$ should allow researchers to analyze an increasing number of microorganism species, but currently, it is still necessary to employ approximations for constructing a generic approach: the effective medium approximation is not sufficient. The first strategy is to approximate the scatterer shape in order to rigorously solve Maxwell's equation. This shape usually corresponds to the equivalent sphere approximation, which allows for straightforward resolution of the scattering problem for the whole range of size parameters by means of standard Lorenz-Mie codes for spheres [30, 44, 45, 46, 47]. Nevertheless, the use of the equivalent sphere approximation can lead to significant errors, especially in research on elongated particles (see [25, 48, 37, 49] and Section 2.2). This is why we are keen on preserving the shape description and are thus compelled to simplify the electromagnetic model of the light-particle interaction. It is a common practice in the field of radiative transfer research to use simplifications corresponding to asymptotic approximations: e.g., the Rayleigh approximation (when size parameters approach zero) and geometrical optics (when size parameters approach infinity). In the case of photosynthetic microorganisms, size parameters are large and refractive-index contrast is low: we are at the soft-particle limit corresponding to the validity range of the anomalous diffraction approximation [34] and Schiff's approximation [50]. These two approximations are identical, except Schiff's approximation involves formulation of the phase functions (which is not the case for anomalous diffraction). We selected Schiff's approximation because it allows us to analyze a great variety of a microorganism's shapes, within the range of size parameters, with accuracy levels that are suitable for the studies of photobioreactors (see [25] regarding comparisons with spectroscopic measurements and [51] regarding comparisons with available reference solutions calculated by the T-Matrix method). Our methodological chain uses efficient code for implementation of Schiff's approximation (including simplification for scattering at a large angle $\theta_{s}$ ), that is available from [51]. This code has been developed for cylindrical and spheroidal homogeneous particles but is based on the Monte Carlo method, which opens up interesting perspectives on the analysis of particles with more complex shapes. Its extension for the analysis of particles with any shape is currently a work in progress. In the case of spheroids and cylinders, using parallel implementation, we observed accuracy levels and CPU times that are compatible with the production of spectral databases needed for the studies on photobioreactors (less than $2 \mathrm{~h}$ for tabulation of radiative properties for 40 wavelengths within PAR, including tabulation of phase functions for 1000 angles) [25, 51]. Accordingly, the combination of the effective medium approximation and Schiff's approximation makes our model numerically tractable. 
The choice of a description in terms of homogeneous-equivalent particles with complex shape (that are analyzed with Schiff's approximation) is pertinent to construction of a generic methodological chain (designed for studies on photobioreactors) that allows for analysis of any photosynthetic microorganism ${ }^{4}$. Nevertheless, in a study on a specific species, rigorous resolution of Maxwell's equation is sometimes tractable, and Schiff's approximation is no longer required. For example, for small microorganisms with modest elongation, most DDA or T-Matrix codes can be used instead of Schiff's approximation. Furthermore, in a study on microorganisms with spherical shape, Lorenz-Mie codes are extremely convenient (see for example [35, 29] and Section 2.2).

The rest of our methodological chain is devoted to determination of the shape, the effective refractive index, and the size distribution of the microbial cells (regarding the distribution of orientations, isotropy is always assumed due to the agitation that is needed for mixing). These parameters must be determined (regardless of the approximation or method that is selected for solving the scattering problem), and we must be able to understand their dependence on the operating mode of the process, by means of either experimentally accessible data (within the scope of the photobioreactor engineering practices) or available databases. Our methodological chain thus implies the following set of characterization procedures (summarized in Table 1) before any implementation of the previously formulated model.

First, the shape and size distributions are determined by optical microscopy and image analysis. Simple rotatory-symmetric parametric shapes are identified and selected in [25, 51], and the size distributions are modeled as $\log$-normal ones for the radius $r_{e q}$ of the volume-equivalent sphere. This is not a restriction: analysis of more complex shapes with Schiff's approximation does not correspond to conceptual difficulties, and analysis of other size distributions is straightforward.

The remaining procedures are designed for determination of the effective refractive index, which reflects the microorganism's internal heterogeneity $[35,36,52]$. First, we determine the imaginary part, which characterizes absorption properties of the continuous medium constituting the microbial cells. For this purpose, we use a model derived from the oceanographic research $[45,46,53,54,55]$. Within the spectral range of PAR, absorption by photosynthetic cells is assumed to be exclusively due to photosynthetic pigments. Moreover, these pigment molecules are diluted enough to be characterized by an in vivo absorption crosssection (independently of the microorganism species and the type of study) and by an internal pigment concentration (which is strongly dependent on the species and culture conditions). We therefore modeled the imaginary part $\kappa_{\nu}$ of the effective refractive index by summing the absorption cross-sections $E_{a, p i g}(\nu)$ of the pigment molecules (expressed in $\mathrm{m}^{2} / \mathrm{kg}$ ), with each of these absorption spectra being weighted by the concentration $C_{p i g}$ of the corresponding pigment species pig within the microorganism in question $\left(C_{\text {pig }}\right.$ is the average local pigment concentration across the cell volume, expressed in $\mathrm{kg} / \mathrm{m}^{3}[25]$ ):

$$
\kappa_{\nu}=\frac{c_{0}}{4 \pi \nu} \sum_{p i g} C_{p i g} E_{a, p i g}(\nu)
$$

where $c_{0}$ is the speed of light in vacuum. Therefore, determination of $\kappa_{\nu}$ implies:

- measurement of the pigment concentrations $C_{\text {pig }}$ for each microorganism species and each culture condition under study: a procedure based on field-tested microbiological protocols is proposed in [25].

- extraction of molecular cross-sections $E_{a, p i g}$ from a database: a database containing data on 14 of the most important photosynthetic pigments in nature (representative pigments of photosynthetic bacteria, cyanobacteria, and microalgae) was constructed in [25] on the basis of the pioneering work of Bidigare and al. [54] and is available in [56].

In Section 2.2, that is dedicated to results, Fig. 4.a represents construction of the $\kappa_{\nu}$ spectrum in the case of Chlamydomonas reinhardtii.

\footnotetext{
${ }^{4}$ Note that the opposite choices are usually made in oceanographic research, during analysis of oceanic albedo. In this case, the backscattered photons have significant effects; therefore, a description of the phytoplankton heterogeneity is required. In order to numerically solve Maxwell's equations for the heterogeneous particles, such models usually simplify the description of the shapes by means of the equivalent sphere approximation (see [30] for an example of core-shell model).
} 
The following procedures deal with determination of the real part $n_{\nu}$ of the effective refractive index. Based on the work of $[47,57,58]$, our methodological chain uses the singly subtractive Kramers-Krönig approximation [59] that yields an expression for $n_{\nu}$ as a function of the spectrum $\kappa_{\nu}$ of the imaginary part and the value $n_{\nu_{p}}$ of the real part at a particular frequency $\nu_{p}$ (see Fig. 4.b in Section 2.2):

$$
n_{\nu}=n_{\nu_{p}}+\frac{2\left(\nu^{2}-\nu_{p}^{2}\right)}{\pi} P \int_{\nu_{\min }}^{\nu_{\max }} \frac{\nu_{1} \kappa_{\nu_{1}}}{\left(\nu_{1}^{2}-\nu^{2}\right)\left(\nu_{1}^{2}-\nu_{p}^{2}\right)} d \nu_{1}
$$

where $\left[\nu_{\min }, \nu_{\max }\right]$ is PAR, and $P$ means that the Cauchy principal value has to be considered for the singularity $\left(\nu_{1}=\nu\right)$. Therefore, what remains is determination of the anchor point $n_{\nu_{p}}$. According to the work of [60] in oceanographic research, we use the Bruggeman mixing rule, which yields the effective refractive index $n_{\nu}$ of a non-absorbing composite particle from the data on the volume fraction and the refractive index of its different structures $[35,36,52]$ :

$$
\sum_{j} f_{j} \frac{\left(\hat{n}_{j, \nu}\right)^{2}-\left(n_{\nu}\right)^{2}}{\left(\hat{n}_{j, \nu}\right)^{2}+2\left(n_{\nu}\right)^{2}}=0
$$

where $f_{j}$ and $\hat{n}_{j, \nu}$ are respectively the volume fraction and the real part of the refractive index for the $j^{t h}$ internal structures of the particle. We chose the anchoring frequency $\nu_{p}$ such that the microorganism under study is non-absorbing at $\nu_{p}\left(i . e ., \kappa_{\nu_{p}}=0\right)$, and $n_{\nu_{p}}$ is determined by solving Eq. 11 for $\nu=\nu_{p}$, where:

- volume fractions $f_{j}$ are measured by electron microscopy and image analysis, or far less frequently, are taken from the literature when available,

- refractive indices $\hat{n}_{j, \nu_{p}}$ of internal anatomic structures are obtained from a small database that is available in [25].

It should be noted that at the current state knowledge, Eq. 11 cannot be used to directly obtain the spectrum $n_{\nu}$ of the refractive index because very little information is available about the spectral properties $\hat{n}_{j, \nu}$ of internal structures. Nonetheless, the choice of non-absorbed anchoring frequency $\nu_{p}$ significantly simplifies the access to these data and allows researchers to estimate the anchor point $n_{\nu_{p}}$.

This methodological chain is summarized in Fig. 3, and the corresponding characterization procedures are listed in Table 1. Further details and a validation procedure that are based on the analysis of spectroscopic data are presented in [25]. 
(1) Determination of the microorganism shape and size distribution by image analysis.

(2) Determination of concentrations of the photosynthetic pigments (protocols available in [25] that are based on the measurement of the pigments dry-mass fraction and the volume fraction of intracellular water).

(3) Construction of the imaginary part of the spectrum of the effective refractive index from the results of (2) and from a database containing in vivo absorption spectra of pigment molecules (the database is available in [56]).

(4) Determination of the volume fractions of the anatomic internal structures by image analysis.

(5) Construction of the real part of the effective refractive index at the anchor point by applying the Bruggeman mixing rule to the results of (4) and the internal-structure real indices obtained from a database (the database is available in [25]).

(6) Construction of the real part of the spectrum of the effective refractive index by applying the singly subtractive Kramers-Krönig approximation to the results of (3) and (5).

Table 1: The main steps of the characterization procedure for determination of input parameters of the model for the radiative properties of a photosynthetic microorganism. 


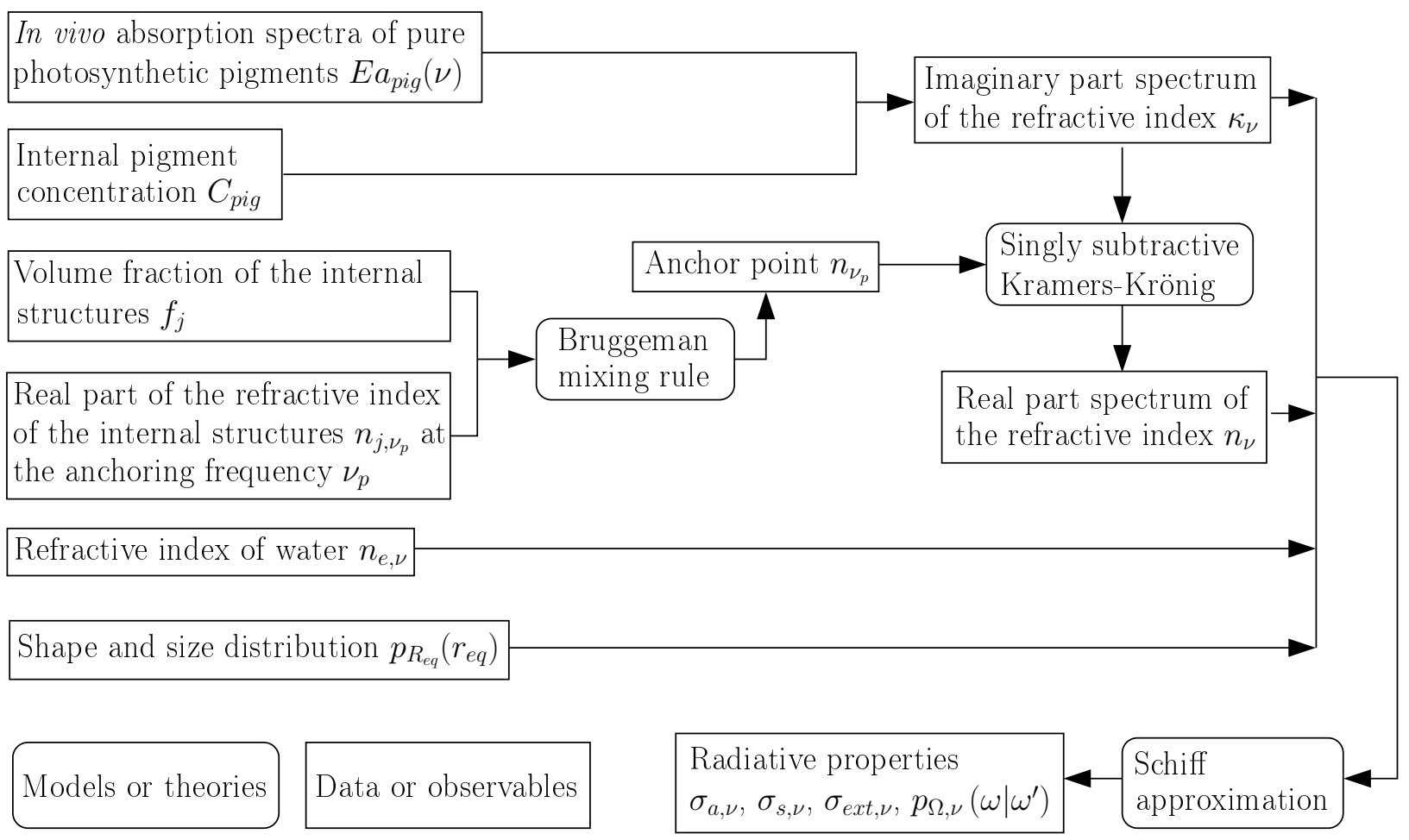

Figure 3: A summary of the methodological chain for determination of radiative properties of a photosynthetic microorganism. 


\subsection{Results}

Figs. 4 and 5 show results obtained by means of the methodological chain presented in Section 2.1 . Among the input parameters of the model, the pigment concentrations are extremely sensitive to the culture conditions. They allow researchers to assess dependence of the radiative properties on the operating mode of the process. In the present case, the parameters have been measured for standard subculture conditions: a shaken $250 \mathrm{~mL}$ Erlenmeyer flask, $100 \mathrm{rpm}$, low photon flux density approximately $30 \mu \mathrm{mol}_{h \nu} \cdot \mathrm{m}^{-2} \cdot \mathrm{s}^{-1}$ $\left(\simeq 7 \mathrm{~W} \cdot \mathrm{m}^{-2}\right)$, and optimal pH and temperature. Fig. 4 presents the effective refractive index obtained by implementing the characterization procedure in Table 1 for $C$. reinhardtii. Note that if different culture conditions are considered, then new parameter values have to be determined by measurement or found in the literature. It should also be noted that spectral variations are usually represented as a function of the wavelength $\lambda_{0}$ of radiation in vacuum. This choice can be quite confusing in the present case where the surrounding medium is water, with refractive index $n_{e, \nu} \neq 1$. Indeed, the scattering problem is affected not by $\lambda_{0}$ but by the wavelength $\lambda$ within the medium (e.g., the size parameter $x$ must be calculated with $\lambda$ ): $\lambda=\frac{\lambda_{0}}{n_{e, \nu}}$. For this reason, we also indicate the frequency $\nu$ of radiation, whose value is identical in vacuum and within the medium: $\nu=\frac{c_{0}}{\lambda_{0}}=\frac{c}{\lambda}$, where the speed of light is $c_{0}$ in vacuum and $c=c_{0} / n_{e, \nu}$ within the medium.

Fig. 5 shows the radiative properties obtained for $C$. reinhardtii and Rhodospirillum rubrum within PAR. C. reinhardtii is a spheroidal unicellular green alga (eukaryote) with PAR ranging from 400 to $700 \mathrm{~nm}$, and Rs. rubrum is a rod-shaped purple bacterium with PAR ranging from 400 to $870 \mathrm{~nm}$. Due to the integration over isotropic orientation distribution in Eq. 8, the single scattering phase function is a function of $\theta_{s}$ only: $p_{\boldsymbol{\Omega}, \nu}\left(\boldsymbol{\omega} \mid \boldsymbol{\omega}^{\prime}\right) \equiv p_{\boldsymbol{\Omega}, \nu}\left(\theta_{s}\right)$, where $\theta_{s}$ is defined in Fig. 2. The phase function of each microorganism is represented for the wavelength at the center of PAR, where scattering is predominant over absorption. We verified that the angular distributions are strongly oriented in forward directions (as discussed in Section 2.1): scattering phase functions are presented on a logarithmic scale for the analysis, but the values obtained indicate that $90 \%$ of the scattered power is within $\theta_{s} \in\left[0,20^{\circ}\right]$, i.e.,

$$
2 \pi \int_{0}^{20^{\circ}} p_{\boldsymbol{\Omega}, \nu}\left(\theta_{s}\right) \sin \left(\theta_{s}\right) d \theta_{s} \simeq 0.9
$$

This situation is similar for all frequencies within PAR, as indicated by the spectral variations of the asymmetry parameter $g$ (see Section 3.2) and the forward scattering fraction $f$ :

$$
g=2 \pi \int_{0}^{\pi} p_{\boldsymbol{\Omega}, \nu}\left(\theta_{s}\right) \cos \left(\theta_{s}\right) \sin \left(\theta_{s}\right) d \theta_{s}
$$

and

$$
f=2 \pi \int_{0}^{\pi / 2} p_{\Omega, \nu}\left(\theta_{s}\right) \sin \left(\theta_{s}\right) d \theta_{s}
$$

where $2 \pi \int_{0}^{\pi} p_{\boldsymbol{\Omega}, \nu}\left(\theta_{s}\right) \sin \left(\theta_{s}\right) d \theta_{s}=1=f+b$, and $b$ is the backscattering fraction. Therefore, for each scattering event, propagation directions of light are predominantly redistributed within a solid angle with aperture $20^{\circ}$. The influence of this redistribution of propagation directions on radiative transfer within photobioreactors is analyzed in Section 3.

Fig. 5 compares (i) the results obtained with an accurate description of the microorganism's shape, subjected to Schiff's approximation (black color) and (ii) the results obtained with the equivalent sphere approximation and the rigorous solution of Maxwell's equation by means of a Lorenz-Mie code (gray color). For $C$. reinhardtii, which has a near-spherical shape, both approaches lead to extremely similar results. This finding confirms that Schiff's approximation yields accurate results if the samples are compared with available reference solutions. The phase functions at $\lambda_{0}=550 \mathrm{~nm}$ are in good agreement, especially for forward scattering, which has a strong influence on radiative transfer within photobioreactors. The discrepancies observed for large angles $\theta_{s}$, where the phase function has small values, and for the asymmetry parameter are both due to the effect of the spheroidal shape of $C$. reinhardtii and the error associated with Schiff's approximation (see [51] for a discussion of scattering at a large angle). These discrepancies are not significant 
when researchers solve the radiative transfer equation [25]. In contrast, the results obtained for Rs. rubrum, which has a cylindrical shape, show significant differences between the scattering properties obtained by the two approaches (e.g., relative difference $\simeq 20 \%$ for the scattering cross-section). On the other hand, the absorption cross-section is less sensitive to the shape of $R s$. rubrum. These results confirm that the equivalent sphere approximation has to be used carefully (or even avoided) when the shape of the microorganism is significantly different from the sphere.

These results are further validated in [25], where the transmittance spectra that were recorded for microorganism suspensions were compared with those predicted by solution of the radiative transfer equation for the radiative properties presented in Fig. 5. In every configuration that has been tested so far, the description of the microorganism's shape increases the accuracy of the results.
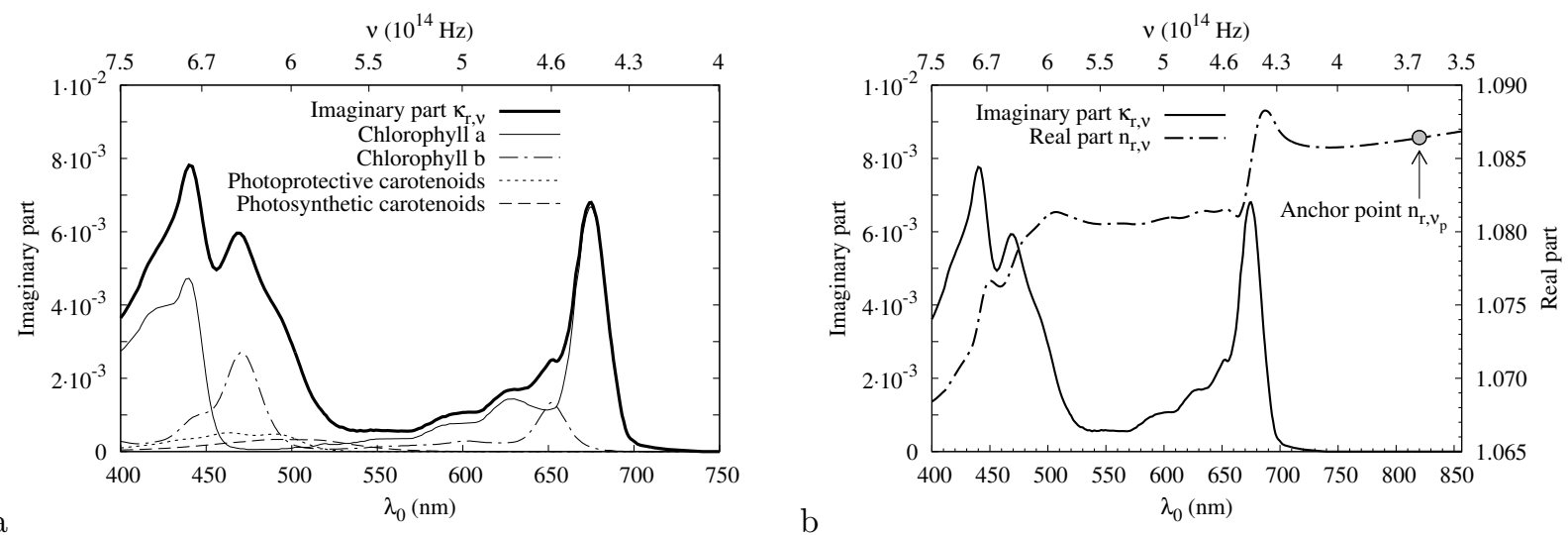

Figure 4: The relative refractive index $m_{r, \nu}=n_{r, \nu}-i \kappa_{r, \nu}$ of the homogeneous equivalent medium for Chlamydomonas reinhardtii as a function of the wavelength $\lambda_{0}$ in vacuum and the frequency $\nu$ of incident radiation. These results were obtained by implementing the characterization procedure summarized in Table 1 . The refractive index $m_{\nu}=n_{\nu}-i \kappa_{\nu}$ is divided by the real index $n_{e, \nu}$ of water: $m_{r, \nu}=m_{\nu} / n_{e, \nu}$, where $n_{e, \nu}$ is calculated by means of an empirical relation reported in [28] (assuming that $n_{e, \nu} \simeq 1.33$ leads to significantly different spectral variations for $n_{r, \nu}$ when $\lambda_{0} \in[400,550 \mathrm{~nm}]$ ). a: The imaginary part $\kappa_{r, \nu}$ obtained with Eq. 9 for the molecular cross-sections $E a_{p i g}$ obtained from the database available in [56] and pigment concentrations $C_{p i g}$ measured by protocols available in [25]: chlorophyll a $677.6 \mathrm{~kg} / \mathrm{m}^{3}$, chlorophyll b $277.2 \mathrm{~kg} / \mathrm{m}^{3}$, photoprotective carotenoids $184.8 \mathrm{~kg} / \mathrm{m}^{3}$, and photosynthetic carotenoids $30.8 \mathrm{~kg} / \mathrm{m}^{3}$. Contribution of each pigment species is also presented. b: The real part $n_{r, \nu}$ produced by the singly subtractive Kramers-Krönig approximation (Eq. 10) for the anchor point $n_{\nu_{p}}=1.44$ at wavelength $\lambda_{0}=820 \mathrm{~nm}\left(\nu_{p}=3.65610^{14} \mathrm{~Hz}\right)$ calculated in [25] with Bruggeman's mixing rule (Eq. 11). 
Chlamydomonas reinhardtii
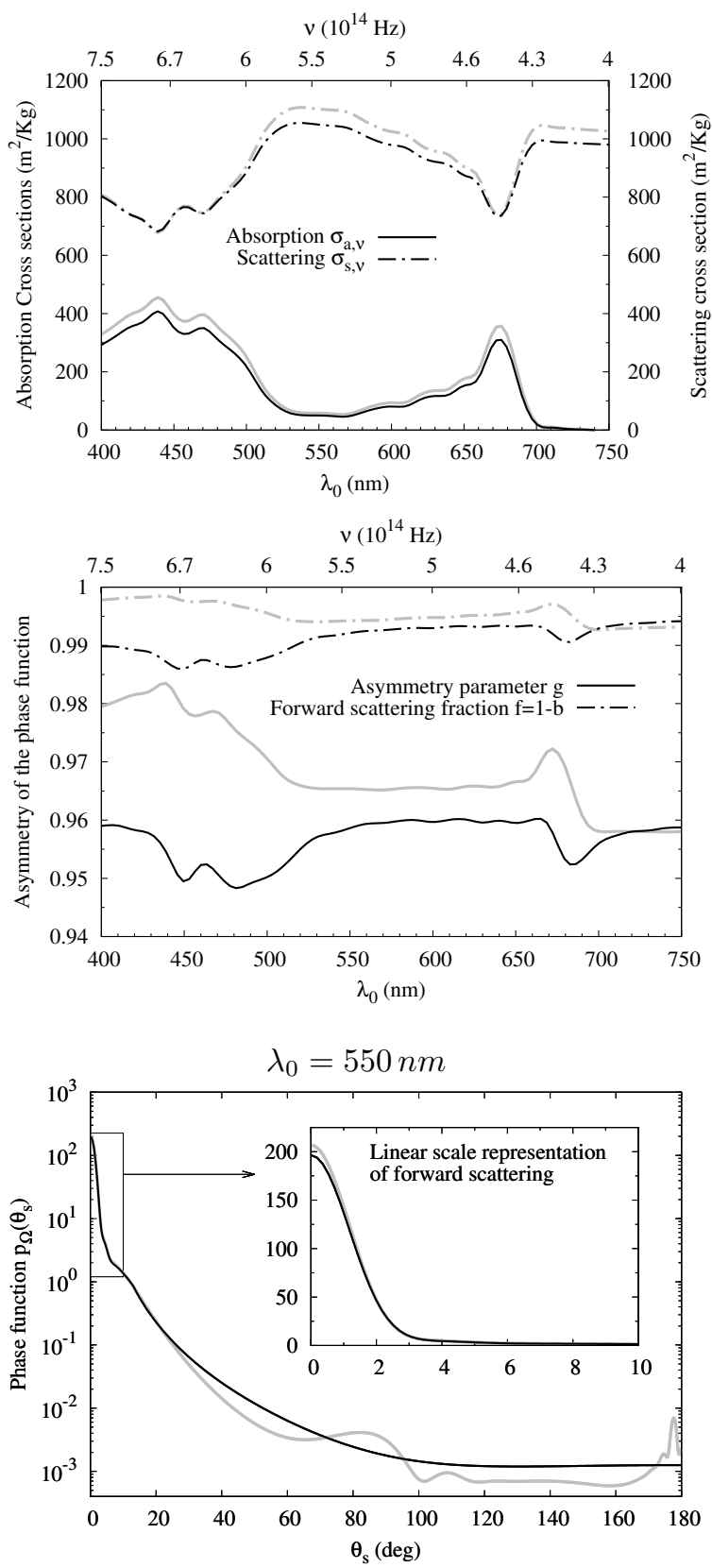

Rhodospirillum rubrum

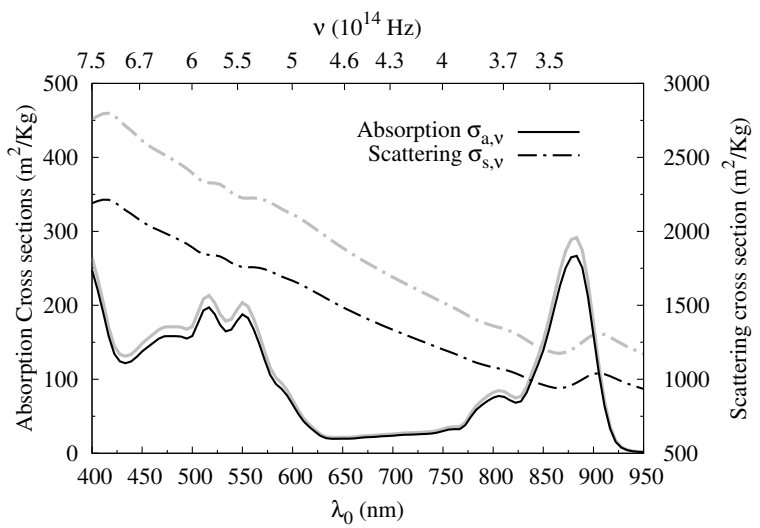

$v\left(10^{14} \mathrm{~Hz}\right)$
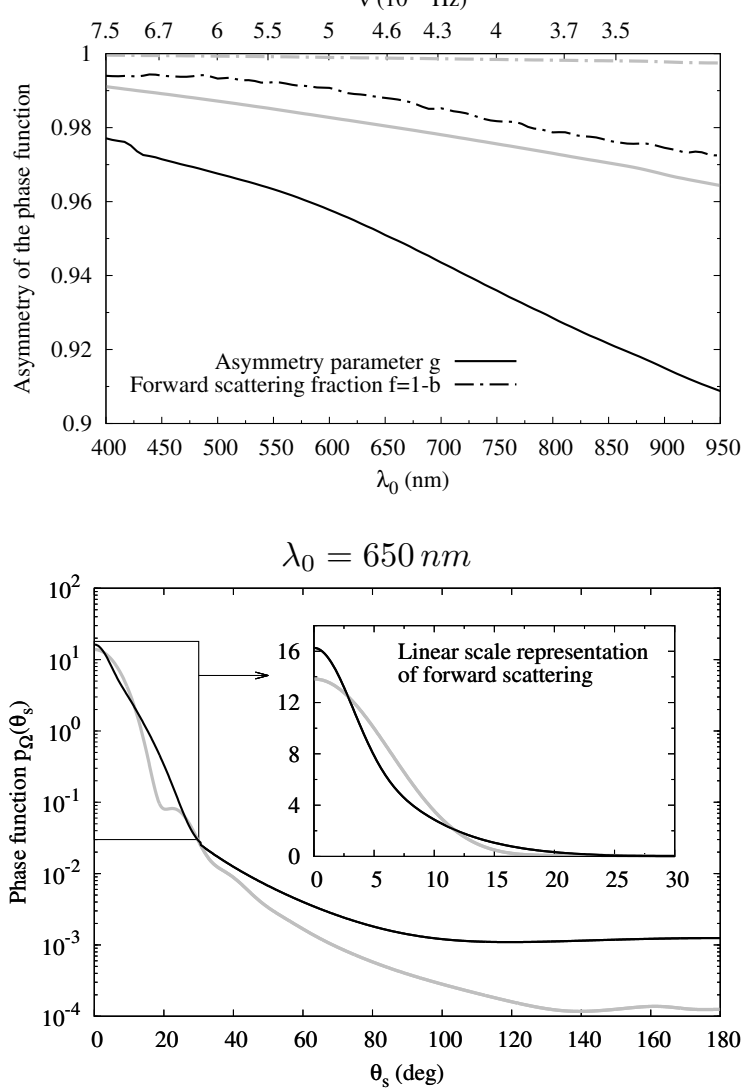

Figure 5: Radiative properties of Chlamydomonas reinhardtii (left) and Rhodospirillum rubrum (right) obtained with the volume equivalent sphere approximation and a Lorentz-Mie code (gray color) and with a more accurate description of the shape and Schiff's approximation (black color): a prolate spheroid with elongation 1.2 (aspect ratio 0.837) for C. reinhardtii and a cylinder with elongation 3.8 (aspect ratio 0.263 ) for $R s$. rubrum. These results were obtained by implementing the methodological chain presented in Section 2.1 for a log-normal size distribution $p_{R_{e q}}\left(r_{e q}\right)=\frac{1}{\sqrt{2 \pi} r_{e q} \ln (s)} e x p\left[-\frac{\left(\ln r_{e q}-\ln \bar{r}_{e q}\right)^{2}}{2 \ln n^{2}(s)}\right]$ with $\bar{r}_{e q}=3.963 \mu \mathrm{m}$ and $s=1.18$ for $C$. reinhardtii, and $\bar{r}_{e q}=0.983 \mu \mathrm{m}$ and $s=1.1374$ for $R s$. rubrum. The refractive index is shown in Fig. 4 for $C$. reinhardtii and in [25] for $R s$. rubrum. The scattering and absorption cross-sections are expressed in $m^{2}$ per $k g$ of dry biomass by means of division of the particulate cross-sections by the effective dry mass $M_{e f f}$ of one microbial cell (see [25]): $M_{\text {eff }}=9.861510^{-14} \mathrm{~kg}$ for $C$. reinhardtii and $M_{\text {eff }}=1.135410^{-15} \mathrm{~kg}$ for $R \mathrm{~s}$. rubrum. In this case, the absorption and scattering coefficients $k_{a, \nu}=C_{x} \sigma_{a, \nu}$ and $k_{s, \nu}=C_{x} \sigma_{s, \nu}$ are obtained with the biomass concentration $C_{x}$ expressed in $\mathrm{kg}$ of dry biomass per $\mathrm{m}^{3}$. 


\subsection{Perspectives}

The accuracy of our model can be improved in many ways, but we believe that solution of the scattering problem is the main obstacle for accurate determination of the radiative properties within photobioreactors. Considering the complexity of shapes, the size parameter range, and the refractive indices of photosynthetic microorganisms, it seems evident that Schiff's approximation should receive increasing attention and consideration in the future, even if the existing exact solutions and numerical methods are continuously improved. Accordingly, the capabilities and limitations of Schiff's approximation are actively studied at present, in particular by comparison with experimental measurements in a single-scattering condition [9], including microwave analog measurements [22].

Another significant challenge for future work is analysis of microorganisms with complex geometric structure. With the Monte Carlo methodology used in [51] for resolution of Schiff's approximation, the geometric calculations required are closely similar to those used in standard geometric-optics codes (i.e., calculation of intersections between rays and surfaces); this situation opens up interesting perspectives on the analysis of particles with complex shape. For example, this approach will enable studies on the effect of the helical shape of Arthrospira platensis, whose radiative properties obtained with a straight cylinder model do not lead to satisfactory spectroscopic validation [25].

Finally, the research into the effect of internal heterogeneity is also an interesting topic for both photobioreactor engineering and natural water/ocean color background analysis [30] (where backscattering is crucial). In order to overcome the difficulty associated with solution of the scattering problem for heterogeneous scatterers, our preliminary studies have been focused on small or spherical microorganisms, but here, the main obstacle is the limited current knowledge about the internal structure of biological cells in terms of the refractive index (this is also a limitation in our method when we calculate the anchor point with a mixing rule).

Despite these areas for improvement, the methodological chain that is presented in the present section already yields radiative properties with a fair level of accuracy for standard culture conditions, when the shape of the microorganism is accurately described (see validation in $[25,51]$ ), including all the spectral and angular data that are needed for formulation of radiative transfer within a photobioreactor. 


\section{Analysis of multiple-scattering radiative transfer within photobioreactors: approximate solutions for the radiation field within one-dimensional Cartesian photobioreactors}

At the end of the previous section, the radiative properties of photosynthetic microorganisms are already available. Therefore, the aim of the following paragraphs is to analyze multiple-scattering radiative transfer in a simple geometric configuration corresponding to a photobioreactor operating close to its optimum. Today, most photobioreactors under study are flat or cylindrical. Here, we chose to focus on the Cartesian one-dimensional radiative configuration of a flat photobioreactor shown in Fig. 6. This study will allow us to derive analytical approximate solutions to the radiative transfer equation. Cylindrical solar photobioreactors will not be discussed here because obtaining an analytical solution for these devices is extremely difficult (for direct solar illumination, the configuration is not one-dimensional). Nonetheless, the stringent analysis of complex geometric structures (including cylindrical solar photobioreactors) will be discussed in Section 4 .

Initially, we will focus on the mesoscopic description associated with the radiative transfer equation. Then, we will introduce the single-scattering approximation and two macroscopic approximations: the P1 approximation and two-flux approximation. All of these discussions are based on the configuration shown in Fig. 6. Collimated emission and Lambertian emission will also be considered in the discussion below; they correspond to the direct component and the diffuse component of solar radiation, respectively. Throughout our study, the biomass concentration $C_{x}$ is homogeneous in the reaction volume $\mathcal{V}$ (assumption of perfect mixing), and the emission phenomena in $\mathcal{V}$ are negligible. The concentration $C_{x}$ is selected close to the optimum for the operation of the photobioreactor: the local photon absorption rate $\mathcal{A}$ at the rear of the photobioreactor is close to the compensation point $\mathcal{A}_{c}$ (see Section 5 and Chapter 5 by Pruvost et al. in this book). 


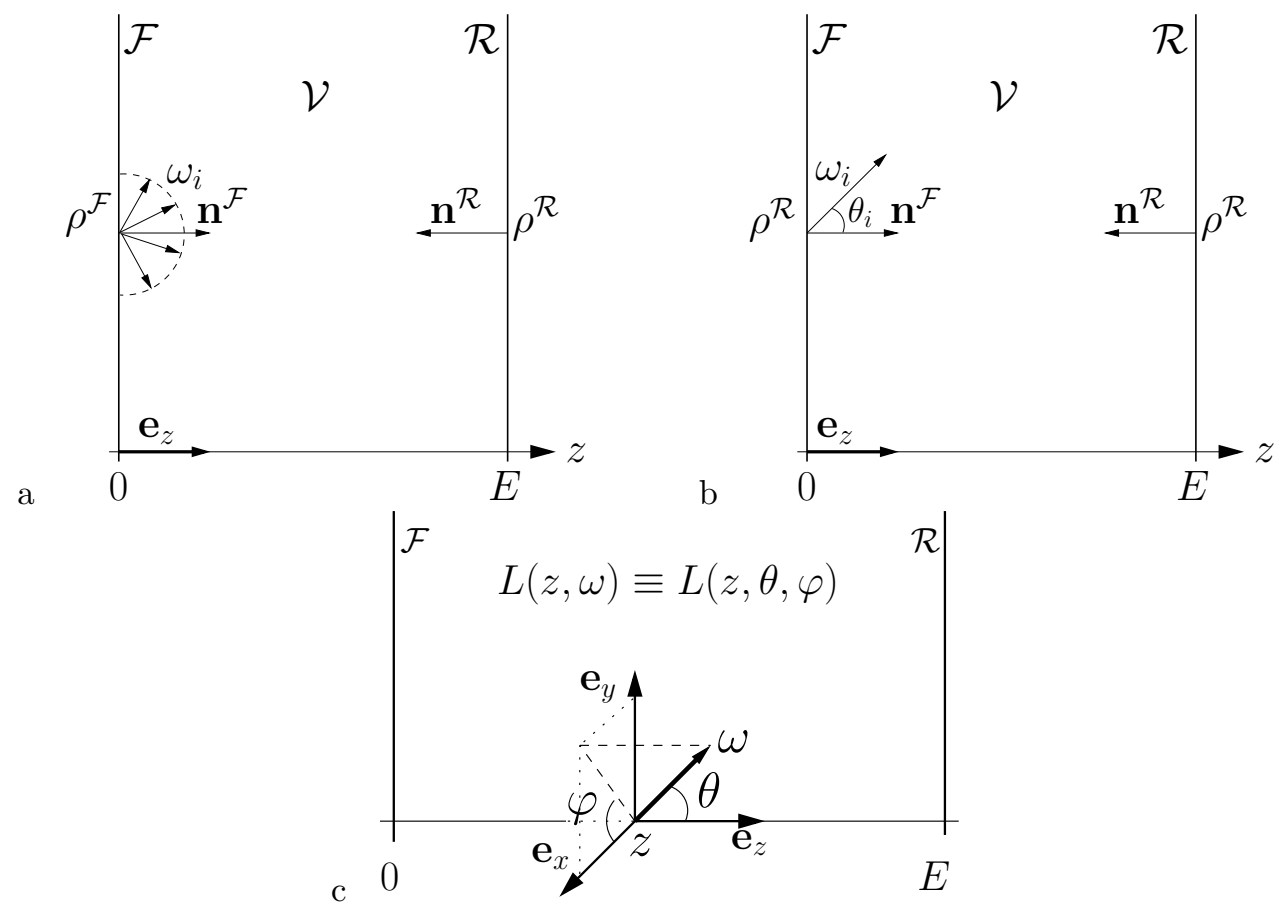

Figure 6: One-dimensional Cartesian radiative configuration that is studied in Section 3. The reaction volume $\mathcal{V}$ is confined by the surfaces $\mathcal{F}$ (front) and $\mathcal{R}$ (rear) located at $z=0$ and $z=E$, respectively. $\rho^{\mathcal{F}}$ and $\rho^{\mathcal{R}}$ are reflectivity values of the surfaces $\mathcal{F}$ and $\mathcal{R} ; \mathbf{n}^{\mathcal{F}}=\mathbf{e}_{z}$ and $\mathbf{n}^{\mathcal{R}}=-\mathbf{e}_{z}$ are their normals. Emission at $\mathcal{F}$ is either a: Lambertian (i.e., diffuse) or b: collimated along the direction $\boldsymbol{\omega}_{i}$, where $\theta_{i}$ is the angle between $\boldsymbol{\omega}_{i}$ and $\mathbf{n}^{\mathcal{F}}$ (the cosine of $\theta_{i}$ is shown as $\mu_{i}=\boldsymbol{\omega}_{i} \cdot \mathbf{n}^{\mathcal{F}}$ ). In both cases, the surface flux density emitted at $\mathcal{F}$ is denoted as $q_{\cap}$. c: Light propagates in all directions $\boldsymbol{\omega}$ (three-dimensional scattering): $\boldsymbol{\omega}=\cos (\varphi) \sin (\theta) \mathbf{e}_{x}+\sin (\varphi) \sin (\theta) \mathbf{e}_{y}+\sin (\theta) \mathbf{e}_{z}$. The element of solid angle is $d \boldsymbol{\omega}=d \varphi d \theta \sin (\theta)$. Within this one-dimensional configuration, for Lambertian incident radiation ( $c f$. a) or for collimated normal incidence (i.e., $\theta_{i}=0$ in b), the intensity is independent of $\varphi$ and is denoted as $L(z, \theta)$ below. The biomass concentration $C_{x}$ within $\mathcal{V}$ is homogeneous. $\mathcal{V}$ is non-emitting. Throughout Section 3, the following configuration is studied: $E=4 \mathrm{~cm}, q_{\cap}=500 \mu \mathrm{mol}_{h \nu} \cdot \mathrm{m}^{-2} \cdot \mathrm{s}^{-1}, C_{x}=0.55 \mathrm{~kg} \cdot \mathrm{m}^{-3}$, $\rho^{\mathcal{F}}=0, \rho^{\mathcal{R}}=0$ or 0.54 (which is close to reflectivity of stainless steel), absorption cross-section $\sigma_{a}=145 \mathrm{~m}^{2} \cdot \mathrm{kg}_{x}^{-1}$, scattering cross-section $\sigma_{s}=922 \mathrm{~m}^{2} \cdot \mathrm{kg}_{x}^{-1}$, and a single scattering phase function with asymmetry parameter $\mathrm{g}=0.945$. These radiative properties were obtained by the method presented in Section 2 for Chlamydomonas reinhardtii; illumination condition being different than those studied in Section 2.2, these properties are different than those presented in Fig. 5. 


\subsection{The radiative transfer equation}

The objective of this section is to introduce basic concepts of the transport theory in participating media (i.e. absorbing and scattering media) and their physical interpretation. These concepts are well established in the radiative transfer research and are detailed in many reference textbooks, such as [61,62,63]. These concepts are not repeated in detail here; they are simply introduced because of their use below for analysis of typical radiative configurations of a photobioreactor. In these typical configurations, the asymmetry parameter of the phase function is close to 1 and optical thickness is intermediate.

The radiative transfer equation is a simplification of the Boltzmann transport equation (developed by Ludwig Boltzmann in 1872 to describe ideal gas of identical particles) made possible by two characteristics of photons as particles:

1. photons all propagate at a locally identical speed: the speed of light $c$ in the medium,

2. they do not interact with each other but interact only with the medium (here, the microorganisms suspension): we are interested in the linear transport phenomenon.

This mesoscopic modeling is a statistical description suitable for complex systems with a large degree of freedom, for example, a set of photons propagating in a scattering medium, fluids, or plasma. This modeling is based on the assumption of repetition of a large number of statistical events within the system. This situation is verified either by the presence of a large number of particles or by replication of a large number of events, for example, scattering events, with a single particle (these two conditions are equivalent in the context of linear transport). The mesoscopic descriptor of the system is the distribution function $f(\mathbf{x}, \boldsymbol{\omega}, t)$, which, up to a normalization factor, is the probability density for a photon to be present at time $t$ and location $\mathbf{x}$ and to have the propagation direction $\boldsymbol{\omega}$. To be precise, $f(\mathbf{x}, \boldsymbol{\omega}, t) d \mathbf{x} d \boldsymbol{\omega}$ is the number of photons within the volume element $d \mathbf{x}$ around the location $\mathbf{x}$, propagating in a direction within the solid-angle element $d \boldsymbol{\omega}$ around $\boldsymbol{\omega}$ (see Fig. 7.a). The system is thus described in six-dimensional space: one dimension for time, three for the geometric space $\mathcal{D}_{\mathbf{x}}$ (which is the reaction volume $\mathcal{V}$ in our study), and two for the propagation directions $\mathcal{D}_{\boldsymbol{\omega}}$, which represent the total solid angle (indicated as $4 \pi$ below). Because all the information about the velocity distribution (or propagation directions) is modeled, Boltzmann-type equations, including the radiative transfer equation, are particularly suitable for description of non-equilibrium situations, even far from equilibrium. We will see that this property is of particular interest in our study because such situations are commonly encountered in photobioreactors. With such mesoscopic description, we can always go back to the usual macroscopic variables, in which only the moments of the velocity distribution are used. For example, the density $\eta(\mathbf{x}, t)$ of photons (the number of photons within the volume element $d \mathbf{x}$, regardless of their propagation direction) is calculated by integrating the distribution function $f$ over all propagation directions:

$$
\eta(\mathrm{x}, t)=\int_{4 \pi} f(\mathbf{x}, \boldsymbol{\omega}, t) d \boldsymbol{\omega}
$$

The radiative transfer equation is the equation of change for the distribution function $f(\mathbf{x}, \boldsymbol{\omega}, t)$ :

$$
\partial_{t} f(\mathbf{x}, \boldsymbol{\omega}, t)+c \boldsymbol{\omega} \cdot \operatorname{grad}_{\mathbf{x}} f(\mathbf{x}, \boldsymbol{\omega}, t)=-c k_{e x t} f(\mathbf{x}, \boldsymbol{\omega}, t)+c k_{s} \int_{4 \pi} f\left(\mathbf{x}, \boldsymbol{\omega}^{\prime}, t\right) p_{\Omega}\left(\boldsymbol{\omega} \mid \boldsymbol{\omega}^{\prime}\right) d \boldsymbol{\omega}^{\prime}
$$

where $c$ is the speed of light in the medium ( $c$ is homogeneous in the context of our study), $\partial_{t}$ is the partial derivative with respect to time $t, \operatorname{grad}_{\mathbf{x}}$ is the gradient with respect to $\mathbf{x}$, and the other parameters are the radiative properties obtained in Section 2 (they are also homogeneous in our study): $p_{\Omega}\left(\boldsymbol{\omega} \mid \boldsymbol{\omega}^{\prime}\right)$ is the phase function, $k_{e x t}=k_{a}+k_{s}$ is the extinction coefficient, with $k_{a}$ and $k_{s}$ the absorption and scattering coefficients, respectively. Temporal variations in the culture conditions that are likely to affect radiative transfer are mainly of two types:

1. variation in incidence and intensity of the solar radiation,

2. changes in the concentration and composition of the biomass, including pigment composition, which strongly influences the radiative properties (see Section 2). 
These transitional states are associated with characteristic periods that are much longer than the characteristic duration of establishment of a steady state for radiative transfer. Therefore, throughout this chapter, we will consider steady-state radiative transfer: the distribution function $f$ is independent of time. This approach does not preclude analysis of temporal relations associated with photon propagation (see Fig. 8). Under these conditions, the radiative transfer equation is written as

$$
c \boldsymbol{\omega} \cdot \operatorname{grad}_{\mathbf{x}} f(\mathbf{x}, \boldsymbol{\omega})=-c k_{e x t} f(\mathbf{x}, \boldsymbol{\omega})+c k_{s} \int_{4 \pi} f\left(\mathbf{x}, \boldsymbol{\omega}^{\prime}\right) p_{\Omega}\left(\boldsymbol{\omega} \mid \boldsymbol{\omega}^{\prime}\right) d \boldsymbol{\omega}^{\prime}
$$



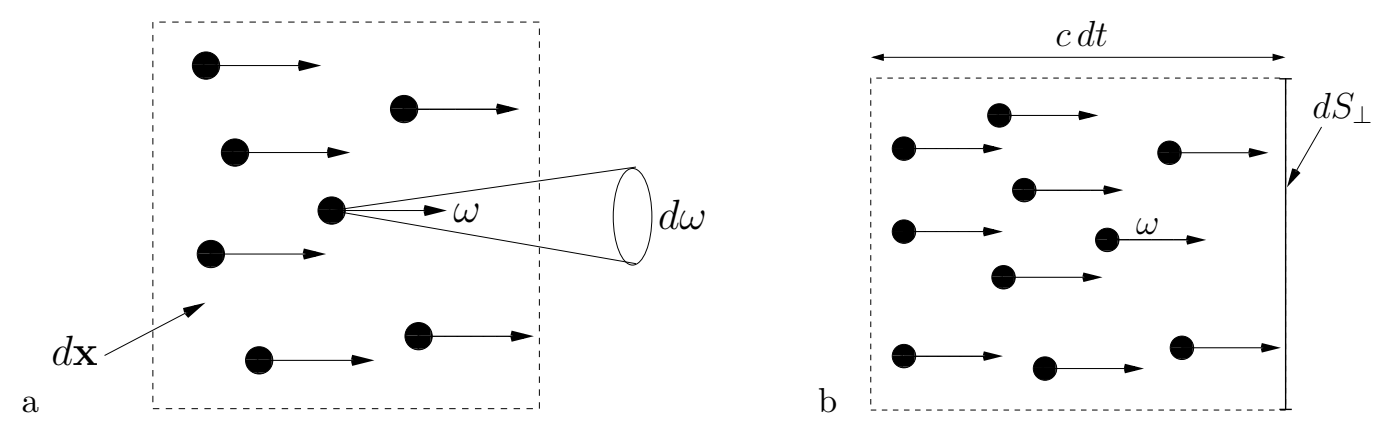

Figure 7: Phase space. a: The volume element of phase space. b: The relation between intensity and the distribution function: The amount of radiant energy that crosses the surface $d S_{\perp}$ during $d t$ is equal to the number of photons propagating in the direction $\boldsymbol{\omega}$ within volume $c d t d S_{\perp}$, multiplied by the energy carried by each photon.
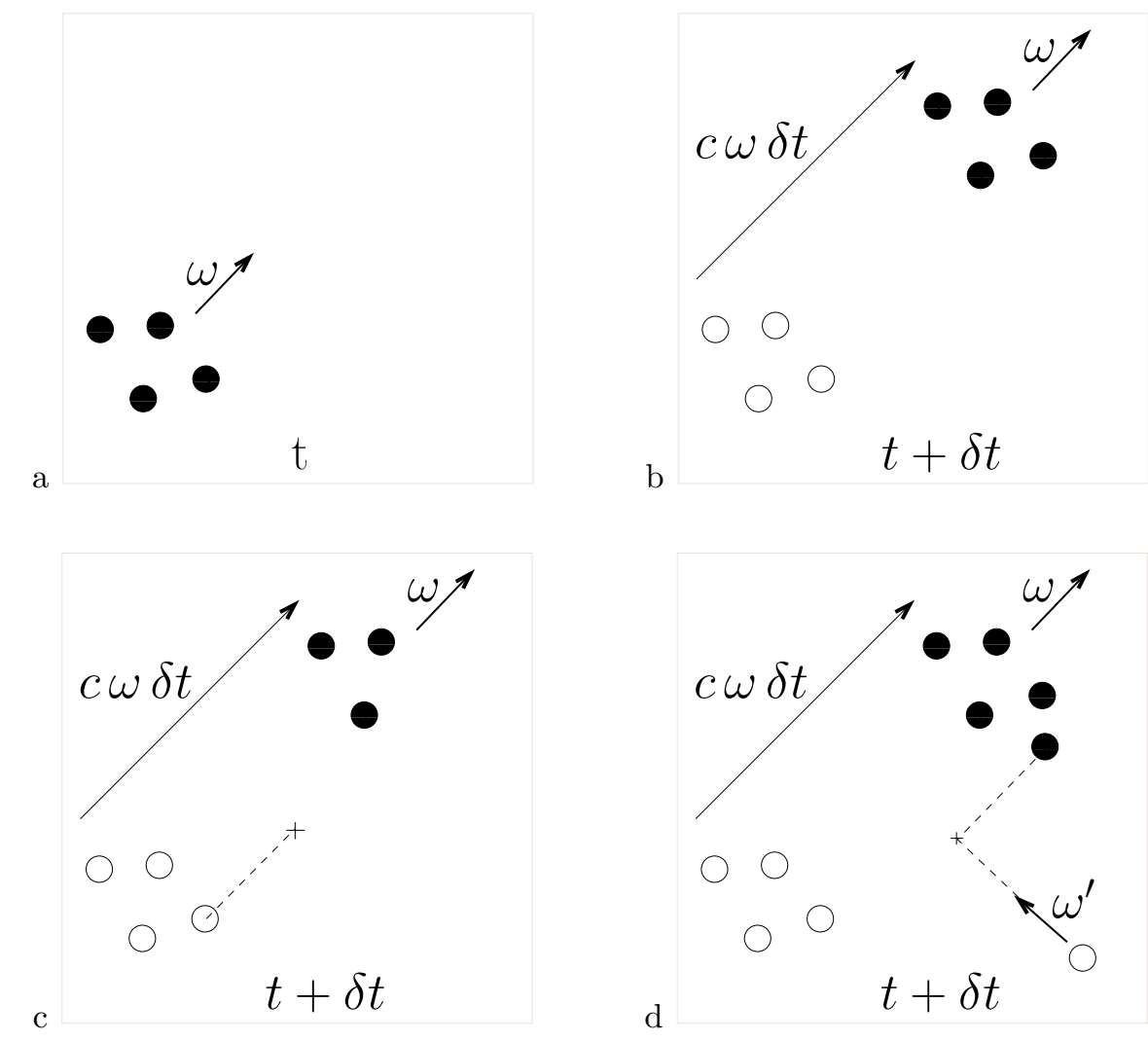

Figure 8: Illustration of a few photons at two time points $t$ and $t+\delta t$, and physical interpretation of the radiative transfer equation. a \& b: The transport term. c: The extinction term. d: The collision term. 
This equation formalizes the balance of the photonic phase in the phase space; this balance is found intuitively in each of its terms. For this purpose, we will follow mentally the propagation of the $f(\mathbf{x}, \boldsymbol{\omega}) d \mathbf{x} d \boldsymbol{\omega}$ photons contained in the phase space volume element $d \mathbf{x} d \boldsymbol{\omega}$ around $(\mathbf{x}, \boldsymbol{\omega})$ during the course of the time interval $\delta t$, as shown in Fig. 8:

- Transport term $c \boldsymbol{\omega} \cdot \operatorname{grad}_{\mathbf{x}} f(\mathbf{x}, \boldsymbol{\omega})$. It indicates variation of $f$ because of free displacement of the photons. The $f(\mathbf{x}, \boldsymbol{\omega}) d \mathbf{x} d \boldsymbol{\omega}$ photons located at $\mathbf{x}$ at time point $t$ have the velocity $c \boldsymbol{\omega}$. In the absence of absorption or scattering, after the time interval $\delta t$, they are located at $\mathbf{x}+c \boldsymbol{\omega} \delta t$. According to Liouville's theorem, the phase space volume $d \mathbf{x} d \boldsymbol{\omega}$ containing the photons is conserved, and we have

$$
f(\mathbf{x}+c \boldsymbol{\omega} \delta t, \boldsymbol{\omega})=f(\mathbf{x}, \boldsymbol{\omega})
$$

If $\delta t$ is a differential element in time, then $f(\mathbf{x}+c \boldsymbol{\omega} \delta t, \boldsymbol{\omega})$ can be expressed as its first-order Taylor expansion around $\mathbf{x}$, which gives us

$$
f(\mathbf{x}, \boldsymbol{\omega})+\left[c \boldsymbol{\omega} \cdot \operatorname{grad}_{\mathbf{x}} f(\mathbf{x}, \boldsymbol{\omega})\right] \delta t=f(\mathbf{x}, \boldsymbol{\omega})
$$

hence

$$
c \boldsymbol{\omega} \cdot \operatorname{grad}_{\mathbf{x}} f(\mathbf{x}, \boldsymbol{\omega})=0
$$

- Extinction term $-c k_{e x t} f(\mathbf{x}, \boldsymbol{\omega})$. It represents the rate at which photons within $d \mathbf{x} d \boldsymbol{\omega}$ are absorbed or scattered in a different direction. They thus leave the phase space volume element under study (see Fig. 8.c). This linear formulation assumes that there is always a scale below which the locations of interaction with microorganisms are distributed randomly and uniformly.

- Collision term $c k_{s} \int_{4 \pi} f\left(\mathbf{x}, \boldsymbol{\omega}^{\prime}\right) p_{\Omega}\left(\boldsymbol{\omega} \mid \boldsymbol{\omega}^{\prime}\right) d \boldsymbol{\omega}^{\prime}$. It represents the source of photons in the phase space volume $d \mathbf{x} d \boldsymbol{\omega}$ in relation to photons with propagation direction $\boldsymbol{\omega}^{\prime}$ that are scattered at $\mathbf{x}$ in the direction $\boldsymbol{\omega}$ (see Fig. 8.d). $c k_{s} f\left(\mathbf{x}, \boldsymbol{\omega}^{\prime}\right) d \mathbf{x} d \boldsymbol{\omega}^{\prime}$ is the rate at which photons within $d \mathbf{x} d \boldsymbol{\omega}^{\prime}$ are scattered, and $p_{\Omega}\left(\boldsymbol{\omega} \mid \boldsymbol{\omega}^{\prime}\right)$ is the probability density for their scattering direction to be $\boldsymbol{\omega}$. Integration over the total solid angle accounts for all incoming directions $\boldsymbol{\omega}^{\prime}$.

This action results in deformation of the distribution function $f$, which is shown in Fig. 9 for the abscissa $z_{0}=3 \mathrm{~cm}$ of the flat-plate photobioreactor in Fig. 6 with collimated normal incidence. Due to the symmetry of the problem (one-dimensional Cartesian configuration), $f$ is a function of only the abscissa $z$ and the angle $\theta$ between the propagation direction $\boldsymbol{\omega}$ and $\mathbf{e}_{z}$. For collimated illumination, if we ignore scattering, then the photons all propagate in the same direction: the incident direction $\boldsymbol{\omega}_{i}$. This situation corresponds to Fig. 9.a where $f\left(z_{0}, \theta\right)$ is zero in all directions except $\boldsymbol{\omega}_{i}$ ( $f$ is a Dirac distribution centered at $\left.\boldsymbol{\omega}_{i}\right)$. When we take into account the scattering by microbial cells, as shown on Fig. 9.b, the propagation directions of the photons gradually deviate as the photons propagate within the suspension: they arrive at $z_{0}$ at different angles of propagation. 


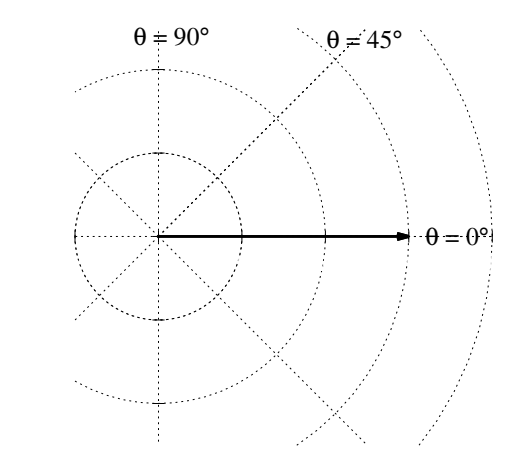

a

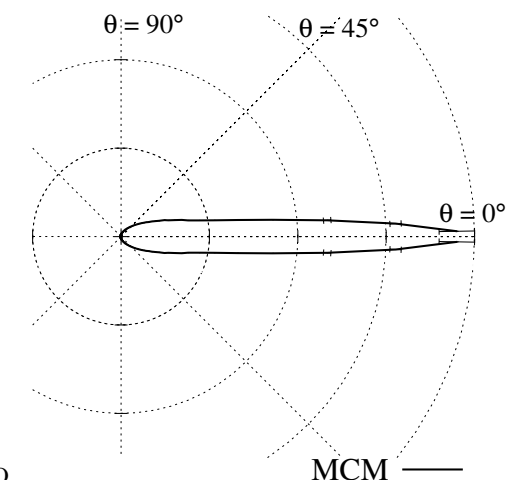

b

Figure 9: The distribution function $f\left(z_{0}, \theta\right)$ at $z_{0}=3 \mathrm{~cm}$ within the one-dimensional photobioreactor shown in Fig. 6 where $\rho^{\mathcal{F}}=\rho^{\mathcal{R}}=0$, for collimated normal incidence $\left(\theta_{i}=0\right)$. a: Without scattering. b: With scattering. The results were obtained with the Monte Carlo method (MCM, see Section 4). 
As we saw in Section 2, the scattering and absorption properties depend on the frequency $\nu$ (or by the same token, on the wavelength $\lambda=c / \nu$ ). It is therefore necessary to distinguish between photons with different frequencies: in addition to $\mathbf{x}$ and $\boldsymbol{\omega}$, the distribution function depends on the variable $\nu$. This functional dependence is usually denoted as $f_{\nu}(\mathbf{x}, \boldsymbol{\omega})$ (rather than $f(\mathbf{x}, \boldsymbol{\omega}, \nu)$ ) to specify its particular characteristics: for elastic scattering (which is the case in our study), no operator in the radiative transfer equation affects the frequency of radiation (only the radiative properties are a function of $\nu$ ). The equations of change for the distribution functions $f_{\nu}$ at each frequency are thus independent:

$$
c \boldsymbol{\omega} \cdot \operatorname{grad}_{\mathbf{x}} f_{\nu}(\mathbf{x}, \boldsymbol{\omega})=-c k_{e x t, \nu} f_{\nu}(\mathbf{x}, \boldsymbol{\omega})+c k_{s, \nu} \int_{4 \pi} d \boldsymbol{\omega}^{\prime} f_{\nu}\left(\mathbf{x}, \boldsymbol{\omega}^{\prime}\right) p_{\Omega, \nu}\left(\boldsymbol{\omega} \mid \boldsymbol{\omega}^{\prime}\right)
$$

In other words, photon populations corresponding to different frequencies evolve completely independently from each other. Nonetheless, the frequency of radiation is a dimension of phase space, just as $\mathbf{x}$ and $\boldsymbol{\omega}$ are. $f_{\nu}(\mathbf{x}, \boldsymbol{\omega}) d \mathbf{x} d \boldsymbol{\omega} d \nu$, within the volume element $d \mathbf{x} d \boldsymbol{\omega}$ around $(\mathbf{x}, \boldsymbol{\omega})$, is the number of photons that have a frequency within the unit interval $d \nu$ around $\nu$. The distribution function $f(\mathbf{x}, \boldsymbol{\omega})$, which describes the photons independently of their frequency, is the integral of $f_{\nu}$ over the spectral range $\left[\nu_{\min }, \nu_{\max }\right]$ under study (PAR in this work):

$$
f(\mathbf{x}, \boldsymbol{\omega})=\int_{\nu_{\min }}^{\nu_{\max }} f_{\nu}(\mathbf{x}, \boldsymbol{\omega}) d \nu
$$

The mesoscopic variable describing radiation in engineering sciences is generally intensity rather than the distribution function. The intensity $L_{\nu}(\mathbf{x}, \boldsymbol{\omega})$ at location $\mathbf{x}$, in the direction $\boldsymbol{\omega}$, and at frequency $\nu$ is expressed as $W \cdot m^{-2} \cdot s r^{-1} \cdot H z^{-1}$. It is the flux density due to photons with direction $\boldsymbol{\omega}$ crossing the surface normal to $\boldsymbol{\omega}$ at location $\mathbf{x}$ (per unit of the solid angle $d \boldsymbol{\omega}$ and per unit of the frequency interval $d \nu$ ). To link the intensity and the distribution function, we will consider the radiant energy $\delta Q$ that crosses the surface $d S_{\perp}$ (perpendicular to $\boldsymbol{\omega}$ ) in the direction $\boldsymbol{\omega}$ during $d t$ :

$$
\delta Q=\int_{\nu_{\min }}^{\nu_{\max }} L_{\nu}(\mathbf{x}, \boldsymbol{\omega}) d \nu d \boldsymbol{\omega} d t d S_{\perp}
$$

which is also equal to the number of photons propagating in the direction $\boldsymbol{\omega}$, with speed $c$, within the volume $d S_{\perp} c d t$ (see Fig. 7.b), multiplied by their energy $h \nu$ :

$$
\delta Q=\int_{\nu_{\min }}^{\nu_{\max }} h \nu \times f_{\nu}(\mathbf{x}, \boldsymbol{\omega}) d \nu d \boldsymbol{\omega} d S_{\perp} c d t
$$

From Eqs. 20 and 21, we obtain the following relation between $f$ and $L$ :

$$
L_{\nu}(\mathbf{x}, \boldsymbol{\omega})=c h \nu f_{\nu}(\mathbf{x}, \boldsymbol{\omega})
$$

Our study of kinetic coupling is based on variables expressed in the number of photons rather than in energy (energetic variables are, for their part, required for formulation of thermodynamic efficiency of the process). Indeed, in a kinetic study, researchers are particularly interested in the flux of photons propagating in the direction $\boldsymbol{\omega}$ at location $\mathbf{x}$; this flux is usually given by $\hat{L}_{\nu}(\mathbf{x}, \boldsymbol{\omega})$ expressed in $\mathrm{mol} \cdot \mathrm{s}^{-1} \cdot \mathrm{m}^{-2} \cdot s r^{-1} \cdot H z^{-1}$ :

$$
\hat{L}_{\nu}(\mathbf{x}, \boldsymbol{\omega})=\frac{L_{\nu}(\mathbf{x}, \boldsymbol{\omega})}{h \nu}=c f_{\nu}(\mathbf{x}, \boldsymbol{\omega})
$$

Despite the different units of measurement, we continue to call $\hat{L}$ intensity. By substituting the above definition into the radiative transfer equation (Eq. 18), we obtain the following equation of change for $\hat{L}$ :

$$
\boldsymbol{\omega} \cdot \operatorname{grad}_{\mathbf{x}} \hat{L}_{\nu}(\mathbf{x}, \boldsymbol{\omega})=-k_{e x t, \nu} \hat{L}_{\nu}(\mathbf{x}, \boldsymbol{\omega})+\alpha_{s, \nu} k_{e x t, \nu} \int_{4 \pi} d \boldsymbol{\omega}^{\prime} \hat{L}_{\nu}\left(\mathbf{x}, \boldsymbol{\omega}^{\prime}\right) p_{\Omega, \nu}\left(\boldsymbol{\omega} \mid \boldsymbol{\omega}^{\prime}\right)
$$

Furthermore, by multiplying Eq. 18 by $h \nu$ and substituting the definition of $L$ (Eq. 22), we obtain the same equation of change for the intensity $L$ (expressed in energy units). Thus, $f, \hat{L}$, and $L$ obey the same radiative transfer equation. 


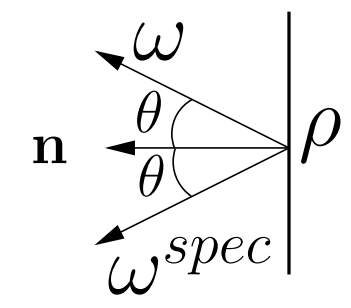

Figure 10: The definition of the specular reflection direction $\boldsymbol{\omega}^{\text {spec }}$ corresponding to the direction $\boldsymbol{\omega}$ for a surface with reflectivity $\rho$ and normal $\mathbf{n}$.

The boundary conditions associated with the radiative transfer equation usually fix the intensity (or the distribution function) for incoming propagation directions. In the context of solar-energy systems, databases contain the incident surface flux density $q_{\cap}$ and its spectral distribution $q_{\cap, \nu}$ (rather than the intensity at the boundary):

$$
q_{\cap}=\int_{\nu_{\min }}^{\nu_{\max }} q_{\cap, \nu} d \nu
$$

In addition to this information, it is necessary to specify angular distribution of the intensity. A Lambertian distribution corresponds to isotropic intensity for the incoming directions (i.e., diffuse incidence)

$$
L_{\nu}(\mathbf{x}, \boldsymbol{\omega})=\frac{q_{\cap, \nu}}{\pi} \quad \text { pour } \boldsymbol{\omega} \cdot \mathbf{n}>0
$$

where $\mathbf{n}$ is the inner normal of the surface. For illumination collimated in the direction $\boldsymbol{\omega}_{i}$, the intensity is zero for all directions within the inner hemisphere, except for $\boldsymbol{\omega}_{i}$

$$
L_{\nu}(\mathbf{x}, \boldsymbol{\omega})=\frac{q_{\cap, \nu}}{\mu_{i}} \delta\left(\boldsymbol{\omega}-\boldsymbol{\omega}_{i}\right) \quad \text { pour } \boldsymbol{\omega} \cdot \mathbf{n}>0
$$

where $\mu_{i}=\cos \left(\theta_{i}\right)$, and $\delta\left(\boldsymbol{\omega}-\boldsymbol{\omega}_{i}\right)$ is the Dirac distribution centered at $\boldsymbol{\omega}_{i}$ (see Fig. 6). Eqs. 26 and 27 set the intensity for the incoming directions only; the intensity for outgoing directions is a result of the radiative transfer problem. Under reflection boundary conditions, the intensity is not fixed, but there is a relation with the intensity across the inner and outer hemispheres. For specular reflection, we have

$$
L_{\nu}(\mathbf{x}, \boldsymbol{\omega})=\rho_{\nu} L_{\nu}\left(\mathbf{x},-\boldsymbol{\omega}^{s p e c}\right) \quad \operatorname{pour} \boldsymbol{\omega} \cdot \mathbf{n}>0
$$

where $\rho_{\nu}$ is surface reflectivity at the frequency $\nu$, and $-\boldsymbol{\omega}^{\text {spec }}$ is the specular direction corresponding to $\boldsymbol{\omega}$ (see Fig. 10). For diffuse reflection, we have

$$
L_{\nu}(\mathbf{x}, \boldsymbol{\omega})=\frac{\rho_{\nu}}{2 \pi} \int_{\boldsymbol{\omega}^{\prime} \cdot \mathbf{n}<0} L_{\nu}\left(\mathbf{x}, \boldsymbol{\omega}^{\prime}\right) d \boldsymbol{\omega}^{\prime} \quad \text { pour } \boldsymbol{\omega} \cdot \mathbf{n}>0
$$

where the outer hemisphere is denoted as $\boldsymbol{\omega}^{\prime} \cdot \mathbf{n}<0$.

As we have seen, the intensity $L$ expressed in energy units and the intensity $\hat{L}$ expressed in kinetic units obey the same radiative transfer equation. The solutions obtained for these two physical quantities thus have the same formulation and, in case of numerical calculation, either of these variables can be determined (with the same formula) depending on the unit chosen for expressing the incident flux $q_{\cap}$ or its spectral distribution $q_{\cap, \nu}$. The latter is an input parameter. If we express $q_{\cap}$ in $W \cdot m^{-2}$, the result determines $L$, but if we express $q_{\cap}$ in $\mu \mathrm{mol} \cdot s^{-1} \cdot m^{-2}$, then the result determines $\hat{L}$. For this reason, we no longer distinguish $L$ and $\hat{L}$ in the rest of this chapter, except when presenting numerical results.

Let us briefly define, according to the intensity, the usual macroscopic radiative quantities used for photobioreactor analysis. Irradiance $G_{\nu}(\mathbf{x})$ is the integral of the intensity over propagation directions:

$$
G_{\nu}(\mathbf{x})=\int_{4 \pi} L_{\nu}(\mathbf{x}, \boldsymbol{\omega}) d \boldsymbol{\omega}
$$


Our study of kinetic-coupling phenomena is based on the specific rate of photon absorption $\mathcal{A}(\mathbf{x})$ (see Sections 1 and 5), that is, the number of photons absorbed by a microbial cell located at $\mathbf{x}$ per unit of the time interval:

$$
\mathcal{A}(\mathbf{x})=\int_{\nu_{\min }}^{\nu_{\max }} \sigma_{a, \nu} G_{\nu}(\mathbf{x}) d \nu
$$

where $G_{\nu}$ is usually expressed in $\mu \mathrm{mol} \cdot \mathrm{s}^{-1} \cdot \mathrm{m}^{-2} \cdot H z^{-1}$ (which is obtained by using $\hat{L}$ rather than $L$ in Eq. 30). Another useful physical quantity is the flux density vector

$$
\mathbf{j}_{R, \nu}(\mathbf{x})=\int_{4 \pi} L_{\nu}(\mathbf{x}, \boldsymbol{\omega}) \boldsymbol{\omega} d \boldsymbol{\omega}
$$

which is used to define the flux density $q_{\nu}$ through any surface with normal $\mathbf{n}: q_{\nu}(\mathbf{x})=\mathbf{j}_{R, \nu}(\mathbf{x}) \cdot \mathbf{n}$. For example, in our one-dimensional configuration of Fig. 6, the surface flux density $q_{\nu}$ along $\mathbf{e}_{z}$ at the abscissa $z$ is

$$
q_{\nu}(z)=\int_{4 \pi} L_{\nu}(\mathbf{x}, \boldsymbol{\omega}) \boldsymbol{\omega} \cdot \mathbf{e}_{z} d \boldsymbol{\omega}
$$

Note that by substituting the boundary condition Eq. 26 or 27 into this definition, one can verify that $q_{\nu}(0)=q_{\cap}+\int_{\boldsymbol{\omega} \cdot \mathbf{e}_{z}<0} L_{\nu}(\mathbf{x}, \boldsymbol{\omega}) \boldsymbol{\omega} \cdot \mathbf{e}_{z} d \boldsymbol{\omega}$, where $\boldsymbol{\omega} \cdot \mathbf{e}_{z}<0$ is the outer hemisphere.

These macroscopic descriptors correspond to integration of the intensity over the space of directions: the irradiance is the 0th moment of the intensity, and the flux density vector is its first moment. This integration results in the loss of a significant portion of information about the propagation directions but reduces the problem to a number of dimensions that is often easier to think about and solve. Most photobioreactor models are based on such variables, as is the case here, with the specific rate of photon absorption $\mathcal{A}$. Rather than solving the mesoscopic model (i.e., the radiative transfer equation) and integrating the intensity afterwards, we are also interested in formulating models that directly address the macroscopic descriptors. Integrating the radiative transfer equation (Eq. 24) over all directions $\boldsymbol{\omega}$, leads to the conservation equation

$$
\operatorname{div} \mathbf{j}_{R, \nu}(\mathbf{x})=-k_{a, \nu} G_{\nu}(\mathbf{x})
$$

In addition to Eq. 34 (that is exact), the construction of macroscopic models consisting of a closed set of equations for the moments of the distribution function (or the intensity), usually requires to formulate approximations. In fluid mechanics, this approximation leads, for example, to the Navier-Stokes equation. The most common approximate macroscopic radiative models describe radiative transfer with heat-like equations (e.g., see the Rosseland approximation and the P1 approximation). Among them, the P1 approximation leads to Fick's equation of the flux density vector $\mathbf{j}_{R, \nu}$ (see Eq. 73); substituting Fick's equation into Eq. 34 yields the following heat-like equation for the irradiance $G$ (in the absence of a source term):

$$
-\tilde{D} \nabla^{2} G_{\nu}(\mathbf{x})=-c k_{a, \nu} G_{\nu}(\mathbf{x})
$$

where $\tilde{D}$ is the macroscopic diffusion coefficient (expressed in $m^{2} / s$ ), $k_{a, \nu}$ is the absorption coefficient (as defined in Section 2), $c$ is the speed of light in the medium, and $\nabla^{2}$ is the Laplacian operator with respect to $\mathbf{x}$. Implementation of such models for the purpose of photobioreactor research will be analyzed in Sections 3.4 and 3.5. These macroscopic descriptions are constructed around situations associated with near-equilibrium conditions. In fluid mechanics, the Knudsen numbers are generally small enough for this hypothesis to be tested, but usually, this is not the case in radiative transfer, in particular in photobioreactors. Nonetheless, the macroscopic approximations allow for analysis based on familiar interpretations (such as diffusion), and their implementation generally corresponds to very short calculation time. Thus, we often seek to reduce radiative problems to those descriptions, even in situations that may seem unsuitable (i.e., far from equilibrium). This practice requires accurate knowledge of the radiative configuration in question. The analysis of typical photobioreactor configurations that we develop in Section 3.2 will allow us to construct, in Section 3.4, a relevant description of the irradiance field on the basis of the P1 approximation (even in far-from-equilibrium situations). 
Note on analysis of the spectral dimension. As we saw in Eq. 18, description of each frequency of the spectrum is completely independent of other frequencies. Thus, all the derivation of equations in the rest of this section, including the approximate solutions developed in Sections 3.3, 3.4, and 3.5, are valid regardless of the wavelength of incident radiation. For this reason, we decided to omit the spectral dependencies in our notations. For numerical calculations, however, one should use the value of the radiative properties and the incident flux $q_{\cap, \nu}$ corresponding to the wavelength in question. In this approach, the radiative transfer equation is solved for each frequency, and the spectral solution thus obtained is integrated over PAR in order to calculate the local absorption rate $\mathcal{A}$ according to Eq. 31. This approach can be implemented with approximate solutions from Sections 3.3, 3.4, and 3.5.

We made a different choice for the numerical applications presented in this section; namely, we chose to obtain "simple" approximate solutions appropriate for analysis. We will use the approximation of an equivalent gray medium, which defines effective radiative properties that are independent of the frequency. These properties are provided in Fig. 6 and were obtained by averaging the spectral properties from Section 2 weighted by the incident spectrum:

$$
\sigma=\frac{\int_{\nu_{\min }}^{\nu_{\max }} q_{\cap, \nu} \sigma_{\nu} d \nu}{q_{\cap}}
$$

and

$$
p_{\Omega}\left(\boldsymbol{\omega} \mid \boldsymbol{\omega}^{\prime}\right)=\frac{\int_{\nu_{\min }}^{\nu_{\max }} q_{\cap, \nu} \sigma_{s, \nu} p_{\Omega, \nu}\left(\boldsymbol{\omega} \mid \boldsymbol{\omega}^{\prime}\right) d \nu}{\sigma_{s} q_{\cap}}
$$

where $\sigma$ is the effective cross-section for extinction $\sigma_{\text {ext }}$, absorption $\sigma_{a}$, or scattering $\sigma_{s} ; q_{\cap}$ and $q_{\cap, \nu}$ are defined in Eq. 25. For this approximation, Eq. 31 becomes $\mathcal{A}(\mathbf{x})=\sigma_{a} G(\mathbf{x})$, where $G$ is the irradiance obtained by direct use of the effective properties in our approximate solutions as well as the flux $q_{\cap}$ rather than $q_{\cap, \nu}$ (no spectral integration is required).

The spectral dimension will be analyzed precisely in Section 4, which is devoted to obtaining reference solutions by the Monte Carlo method.

\subsection{Optical thickness and invariance of the transport problems}

In this section, we introduce a set of dimensionless quantities commonly used to characterize radiative transfer in specific configurations. These quantities are necessary for a detailed understanding of radiative transfer in photobioreactors. Depending on the value of the albedo $\alpha_{s}$, the asymmetry parameter $g$ of the phase function, and the optical thickness of the medium, it is possible to conduct first analysis of the scattering regime and to identify the appropriate approximations.

The single-scattering albedo represents the proportion of the interaction events that are scattering events:

$$
\alpha_{s}=\frac{k_{s}}{k_{e x t}}
$$

where $k_{e x t}=k_{s}+k_{a} . \alpha_{s}$ is equal to 0 in the case of a purely absorbing medium (i.e., $\left.k_{s}=0\right)$ and equal to 1 in a purely scattering medium $\left(i . e ., k_{a}=0\right)$. In the radiative configuration of the flat-plate photobioreactor in Fig. 6, $\alpha_{s} \simeq 0.86$ means that when a photon interacts with a microorganism, the photon is scattered with probability 0.86 and absorbed with probability 0.14 . Each scattering event statistically redistributes the propagation direction of the photon according to the single-scattering phase function ${ }^{5} p_{\boldsymbol{\Omega}}\left(\boldsymbol{\omega} \mid \boldsymbol{\omega}^{\prime}\right)$. For analysis purposes, it is interesting to reduce this angular information to its first moment: the asymmetry parameter

$$
g=\int_{4 \pi} p_{\boldsymbol{\Omega}}\left(\boldsymbol{\omega} \mid \boldsymbol{\omega}^{\prime}\right) \boldsymbol{\omega} \cdot \boldsymbol{\omega}^{\prime} d \boldsymbol{\omega}
$$

\footnotetext{
${ }^{5} p_{\boldsymbol{\Omega}}\left(\boldsymbol{\omega} \mid \boldsymbol{\omega}^{\prime}\right) d \boldsymbol{\omega}$ is the probability that when a scattering event occurs, a photon with the propagation direction $\boldsymbol{\omega}^{\prime}$ is scattered within the element of solid angle $d \boldsymbol{\omega}$ around the direction $\boldsymbol{\omega}$. In the present context, a local thermodynamic equilibrium can be assumed; therefore, $p_{\boldsymbol{\Omega}}\left(\boldsymbol{\omega} \mid \boldsymbol{\omega}^{\prime}\right)=p_{\boldsymbol{\Omega}^{\prime}}\left(\boldsymbol{\omega}^{\prime} \mid \boldsymbol{\omega}\right)$.
} 
where $\boldsymbol{\omega} \cdot \boldsymbol{\omega}^{\prime}=\cos \left(\theta_{s}\right)$, with the notations shown in Fig. 2. $g$ ranges from -1 to 1 and is equal to 0 when the phase function is symmetric for forward and backward directions (i.e., for $\boldsymbol{\omega} \cdot \boldsymbol{\omega}^{\prime}>0$ and $\left.\boldsymbol{\omega} \cdot \boldsymbol{\omega}^{\prime}<0\right)$. This is the case, for example, for an isotropic phase function: all the scattering directions are equiprobable. Furthermore, $g<0$ in the case of a phase function oriented in backward directions, and $g>0$ for a phase function oriented in forward directions. In the case of photosynthetic microorganisms, $g$ is close to 1 , or more precisely, $g=0.945$ in the situation under study (see Fig. 6). This means that in a photobioreactor, each scattering event predominantly redistributes the propagation direction within a small solid angle around the incident direction $\boldsymbol{\omega}^{\prime}$ (aperture $\simeq 20^{\circ}$, see Section 2.2). Nevertheless, the sum of these successive scattering events may lead to a significant deviation in the propagation directions (see Figs. 9 and 11) and may result in, among other things, a complex residence time distribution $[64,65]$. The information about this distribution is commonly reduced to the scattering optical thickness $e_{s}$, which is the product of the characteristic dimension of the reaction volume ( $E$ in the case of our flat-plate photobioreactor in Fig. 6) and the scattering coefficient:

$$
e_{s}=E k_{s}
$$

$e_{s}$ is the inverse of the Knudsen number. In the radiative configuration under study, $e_{s} \simeq 20$. 

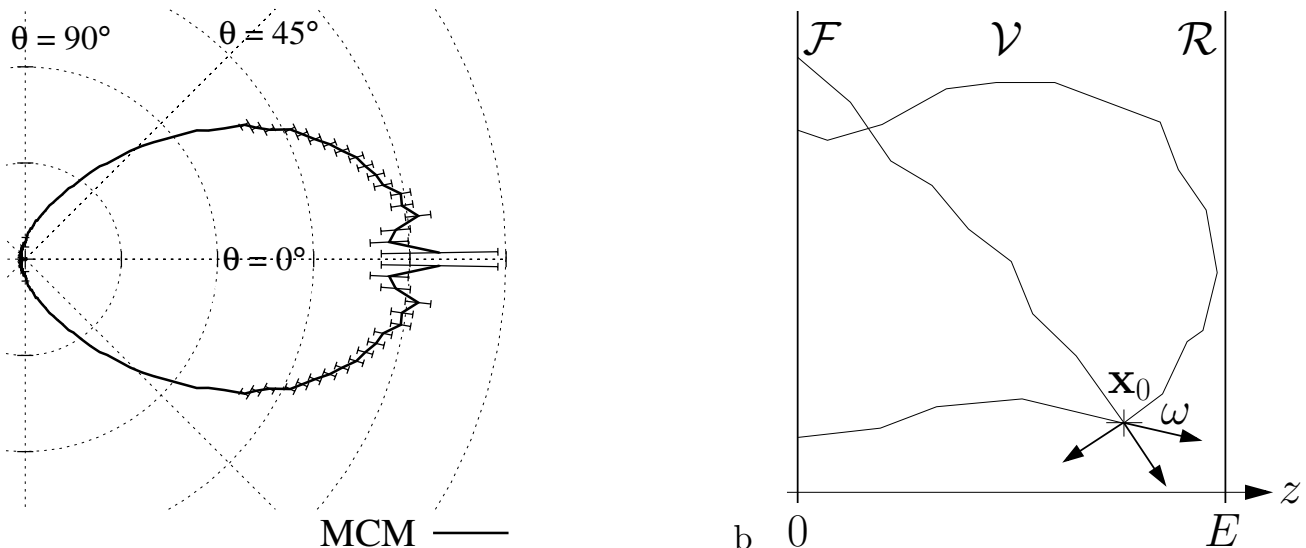

Figure 11: a: Angular distribution of the intensity $L\left(z_{0}, \theta\right)$ at the location $z_{0}=3 \mathrm{~cm}$ within the one-dimensional photobioreactor from Fig. 6 with Lambertian emission and $\rho^{\mathcal{F}}=\rho^{\mathcal{R}}=0$. The results were obtained by the Monte Carlo method (see Section 4). b: Assumption for the corresponding optical paths $\left(z_{0}\right.$ is the abscissa of the location $\mathbf{x}_{0}$, and $\theta$ is the angle between $\boldsymbol{\omega}$ and $\mathbf{e}_{z}$ ). The complex angular distribution of the intensity is due to the special shape of the phase function for photosynthetic microorganisms. The multiple-scattering optical path in question is the result of many scattering events corresponding to a small deviation of the propagation direction. Among them, those leading to directions $\boldsymbol{\omega}$ that are significantly different from $\mathbf{e}_{z}$ are the longest: the probability for absorption to occur along these paths before reaching $\mathbf{x}_{0}$ is high (regarding attenuation by absorption along optical paths, see Section 4.1). 
During analysis of radiative transfer, the angular distribution of the intensity is of great significance because its deviation from isotropy defines the validity conditions of various approximations and physical interpretations. Here, it is crucial to distinguish (i) the angular distribution of the phase function, which corresponds to redistribution of propagation directions because of a single scattering event (under the assumption of perfect mixing, this radiative property of the microbial cells is homogeneous within the photobioreactor) and (ii) the angular distribution of the intensity, which corresponds to the distribution of the propagation directions at a given location. The angular distribution of the intensity (which is generally a function of the location within the photobioreactor) results from multiple scattering events: it is formulated by solving the radiative transfer equation. Because the asymmetry parameter is $\simeq 0.95$, it is a significant obstacle for analysis of the angular distribution of intensity within a photobioreactor. Indeed, in this situation, the extent to which the successive forward-scattering events redistribute the propagation directions is difficult to grasp. To simplify the physics involved, it is customary to use a transport problem equivalent to that under study but where the phase function is isotropic. Analysis of this equivalent problem is much easier because information about the initial propagation direction of a photon (i.e., the boundary conditions) is lost from the first scattering event: the propagation direction $\omega$ is redistributed isotropically, independently of the incoming direction $\boldsymbol{\omega}^{\prime}$. This equivalent problem is derived by replacing the radiative properties $k_{e x t}, k_{s}, k_{a}$, and $p_{\boldsymbol{\Omega}}$ obtained in Section 2 by the new radiative properties $k_{e x t}^{*}, k_{s}^{*}, k_{a}^{*}$, and $p_{\boldsymbol{\Omega}}^{*}$, according to the following transformation:

$$
\begin{aligned}
k_{\text {ext }}^{*} & =k_{\text {ext }}\left(1-\alpha_{s} g\right) \\
k_{s}^{*} & =k_{s}(1-g) \\
k_{a}^{*} & =k_{a} \\
p_{\Omega}^{*} & =\frac{1}{4 \pi} \quad \text { (isotropic phase function) }
\end{aligned}
$$

The dimensionless quantities defined previously become

$$
\begin{gathered}
\alpha_{s}^{*}=\alpha_{s} \frac{1-g}{1-\alpha_{s} g} \\
g^{*}=0 \\
e_{s}^{*}=e_{s}(1-g)
\end{gathered}
$$

The radiative transfer equation is not invariant with this transformation, but we find this invariance in various situations: for example, the diffusion equation obtained with the $\mathrm{P} 1$ approximation is invariant with this transformation (see Section 3.4). In addition, we found that solution of this equivalent problem usually provides results that are very close to those obtained by solution of the original problem in the case of a photobioreactor. The approximate solutions that are derived and validated in Sections 3.3 and 3.4 are obtained by addressing this equivalent problem. Note that this transformation is also useful for comparison of very different situations, regardless of the form of the phase function: in the field of transport theory research, when mentioning optical thickness, we are generally referring to $e_{s}^{*}$ rather than $e_{s}$.

In the radiative configuration shown in Fig. $6, \alpha_{s}^{*}=0.25$ and $e_{s}^{*}=1.1$; this situation is typical of a photobioreactor operating at its optimum biomass production rate. Such intermediate values of optical thickness mean that scattering plays a significant role but does not systematically ensure that the intensity within the medium is close to isotropy. Accordingly, the angular distribution within the reaction volume depends on both the scattering phenomenon and the boundary conditions. Although analysis of such intermediate situation is not straightforward, the equivalent problem brings us back to situations that are easily manipulated. Instead of reasoning about complex optical paths resulting from multiple forwardscattering events (as in Fig. 11), in the following section, we use the single-scattering approximation, where photons suffer zero or one isotropic scattering event only (see Figs. 12 and 13). 


\subsection{The single-scattering approximation}

It is always possible to formulate the intensity $L$ of the entire photon population as the sum of the intensity values $L^{(j)}$ corresponding to the photons that have undergone exactly $j$ scattering events:

$$
L=L^{(0)}+L^{(1)}+L^{(2)}+L^{(3)}+\ldots
$$

This simply means that the total number of photons within any phase space volume element $d \mathbf{x} d \boldsymbol{\omega}$ is the sum of the photons that have undergone $j$ diffusion events. Each $L^{(j)}$ is governed by an equation of its own, in which the source term corresponds to the lower-order photons $L^{(j-1)}$ that are scattered locally and move from population $(j-1)$ to population $(j)$. In this section, we first derive the corresponding infinite system of coupled equations (Eq. 46 and Eq. 54) and the associated boundary conditions (Eqs. 47-48 and Eqs. 56-57) in the special case of the one-dimensional photobioreactor in Fig. 6. Then, we will find a solution to the equivalent transport problem presented in Section 3.2, where scattering optical thickness is $e_{s} \simeq 1$. In this situation, the single scattering approximation [66] is relevant: only the scattering orders (0) and (1) will be selected, and higher orders will be ignored

$$
L \simeq L^{(0)}+L^{(1)}
$$

This approach significantly simplifies solution of the mesoscopic problem. Thus, we consider only two subsets in the photon population: the photons that arrive directly from the surface $\mathcal{F}$ (did not undergo any scattering events) will be called "ballistic photons", and the photons that have undergone only one scattering event will be called the "scattered photons".

\subsubsection{Expansion of the radiative transfer equation into the successive order of scattering}

This section is focused on our one-dimensional photobioreactor with collimated incidence at $z=0$, in the absence of reflection. Ballistic photons obey an independent radiative transfer equation without a source term; the source is at the boundary $\mathcal{F}$ only:

$$
\boldsymbol{\omega} \cdot \operatorname{grad}_{\mathbf{x}} L^{(0)}(\mathbf{x}, \boldsymbol{\omega})=-k_{e x t} L^{(0)}(\mathbf{x}, \boldsymbol{\omega})
$$

with the following boundary conditions (according to Section 3.1):

- At $z=0$,

$$
L^{(0)}(\mathbf{x}, \boldsymbol{\omega})=\frac{q_{\cap}}{\mu_{i}} \delta\left(\boldsymbol{\omega}-\boldsymbol{\omega}_{i}\right) \quad \text { for } \mathbf{x} \in \mathcal{F}, \boldsymbol{\omega} \cdot \mathbf{n}^{\mathcal{F}}>0
$$

where $\mu_{i}=\cos \left(\theta_{i}\right)$, and $\delta\left(\boldsymbol{\omega}-\boldsymbol{\omega}_{i}\right)$ is the Dirac distribution centered at $\boldsymbol{\omega}_{i}$ (see Fig. 6).

- At $z=E$,

$$
L^{(0)}(\mathbf{x}, \boldsymbol{\omega})=0 \quad \text { for } \mathbf{x} \in \mathcal{R}, \boldsymbol{\omega} \cdot \mathbf{n}^{\mathcal{R}}>0
$$

The solution for $L^{(0)}$ is straightforward: it is the incident intensity $\frac{q_{\cap}}{\mu_{i}}$ attenuated by Bouguer's exponential extinction along the ballistic trajectory

$$
L^{(0)}(\mathbf{x}, \boldsymbol{\omega})=\frac{q_{\cap}}{\mu_{i}} \exp \left(-k_{e x t} \frac{z}{\mu_{i}}\right) \delta\left(\boldsymbol{\omega}-\boldsymbol{\omega}_{i}\right)
$$

where $z$ is the abscissa of the location $\mathbf{x}$.

$L^{(1)}$ obeys the following radiative transfer equation:

$$
\boldsymbol{\omega} \cdot \operatorname{grad}_{\mathbf{x}} L^{(1)}(\mathbf{x}, \boldsymbol{\omega})=-k_{\text {ext }} L^{(1)}(\mathbf{x}, \boldsymbol{\omega})+\mathcal{C}^{(0)}(\mathbf{x}, \boldsymbol{\omega})
$$

where $\mathcal{C}^{(0)}$ is the source term accounting for ballistic photons scattered at $\mathbf{x}$, which then arrive into the population (1) with the direction $\boldsymbol{\omega}$ (according to the collision term of the radiative transfer equation in Section 3.1):

$$
\mathcal{C}^{(0)}(\mathbf{x}, \boldsymbol{\omega})=\alpha_{s} k_{e x t} \int_{4 \pi} d \boldsymbol{\omega}^{\prime} L^{(0)}\left(\mathbf{x}, \boldsymbol{\omega}^{\prime}\right) p_{\Omega}\left(\boldsymbol{\omega} \mid \boldsymbol{\omega}^{\prime}\right)
$$

where $\alpha_{s} k_{e x t}=k_{s}$. The boundary conditions for $L^{(1)}$ are as follows: 
- At $z=0$,

$$
L^{(1)}(\mathbf{x}, \boldsymbol{\omega})=0 \quad \text { for } \mathbf{x} \in \mathcal{F}, \boldsymbol{\omega} \cdot \mathbf{n}^{\mathcal{F}}>0
$$

- At $z=E$,

$$
L^{(1)}(\mathbf{x}, \boldsymbol{\omega})=0 \quad \text { for } \mathbf{x} \in \mathcal{R}, \boldsymbol{\omega} \cdot \mathbf{n}^{\mathcal{R}}>0
$$

The incoming intensity is equal to zero because on the one hand, there is no emission at the boundaries for this population (only ballistic photons are emitted at the boundary $\mathcal{F}$ ), and on the other hand, reflectivity of $\mathcal{F}$ and $\mathcal{R}$ is zero in the present case.

For the $j$ th order, we have

$$
\boldsymbol{\omega} \cdot \operatorname{grad}_{\mathbf{x}} L^{(j)}(\mathbf{x}, \boldsymbol{\omega})=-k_{e x t} L^{(j)}(\mathbf{x}, \boldsymbol{\omega})+\mathcal{C}^{(j-1)}(\mathbf{x}, \boldsymbol{\omega})
$$

where

$$
\mathcal{C}^{(j-1)}(\mathbf{x}, \boldsymbol{\omega})=\alpha_{s} k_{e x t} \int_{4 \pi} d \boldsymbol{\omega}^{\prime} L^{(j-1)}\left(\mathbf{x}, \boldsymbol{\omega}^{\prime}\right) p_{\Omega}\left(\boldsymbol{\omega} \mid \boldsymbol{\omega}^{\prime}\right)
$$

is the source term corresponding to the transitions from population $(j-1)$ to population $(j)$ because of scattering (with $\mathcal{C}^{(0)}=0$ ). The boundary conditions are as follows:

- At $z=0$,

$$
L^{(j)}(\mathbf{x}, \boldsymbol{\omega})=0 \quad \text { for } \mathbf{x} \in \mathcal{F}, \boldsymbol{\omega} \cdot \mathbf{n}^{\mathcal{F}}>0
$$

- At $z=E$,

$$
L^{(j)}(\mathbf{x}, \boldsymbol{\omega})=0 \quad \text { for } \mathbf{x} \in \mathcal{R}, \boldsymbol{\omega} \cdot \mathbf{n}^{\mathcal{R}}>0
$$

The equation for $L^{(0)}$ is independent of the other equations, and each of the higher orders $j>0$ is coupled only to the order $j-1$ : this system of equations is closed at the 0th order. Therefore, truncation of the expansion Eq. 44 involves simply ignoring the existence of certain photons; this approach will not cause an error in the description of the orders that are selected for analysis. 

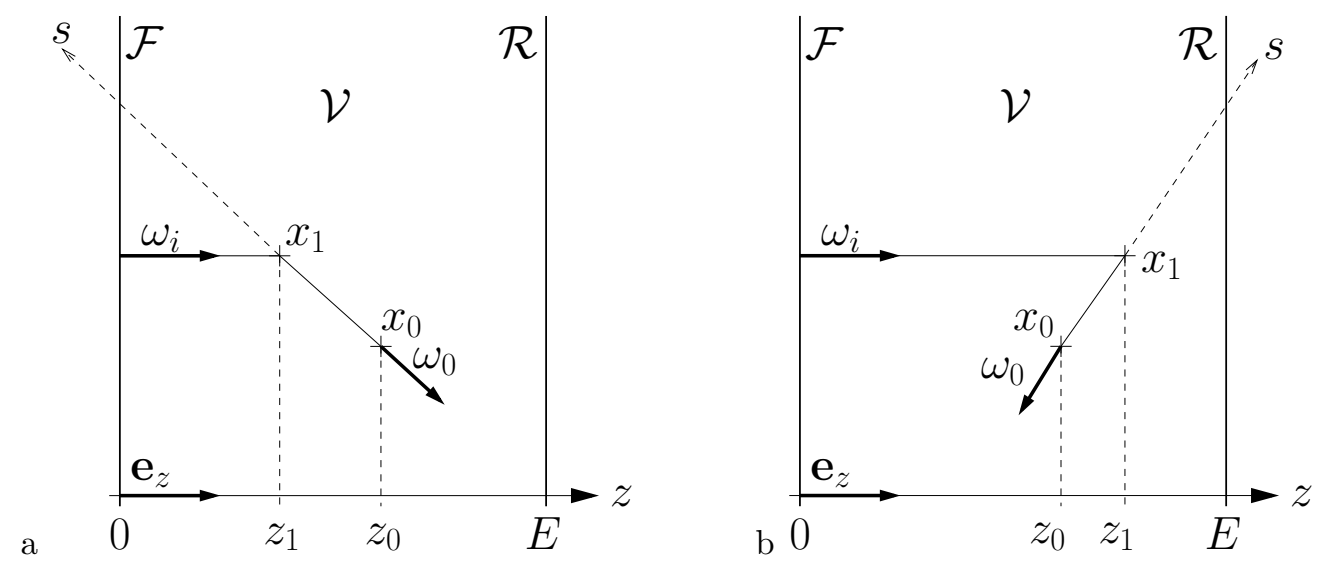

Figure 12: Single-scattering optical paths contributing to $L^{(1)}\left(z_{0}, \boldsymbol{\omega}_{0}\right)$. a: $\mu_{0}>0$; b: $\mu_{0}<0$, where $\mu_{0}=\boldsymbol{\omega}_{0} \cdot \mathbf{e}_{z}$.

a
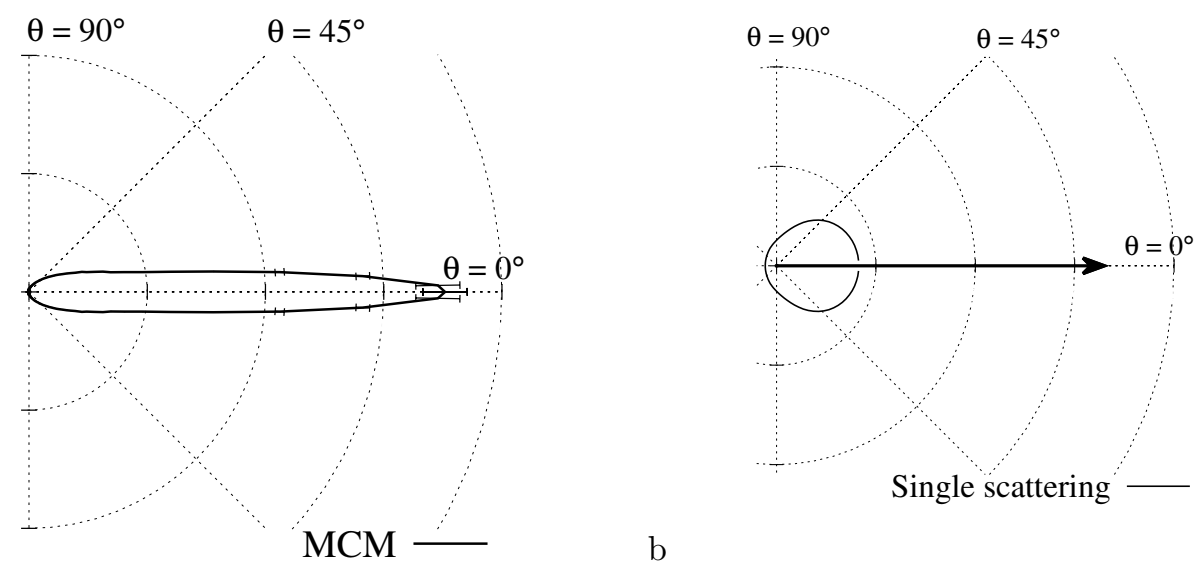

$\mathrm{b}$

Figure 13: Angular distribution of the intensity $L\left(z_{0}, \theta\right)$ at location $z_{0}=3 \mathrm{~cm}$ within the one-dimensional photobioreactor shown in Fig. 6: $\rho^{\mathcal{F}}=\rho^{\mathcal{R}}=0$, collimated normal incidence $\mu_{i}=1$. a: Results obtained by the Monte Carlo method (see Section 4). b: Results obtained for the equivalent transport problem where $\alpha_{s}^{*}=0.25, k_{e x t}^{*}=110 \mathrm{~m}^{-1}$, and $p_{\Omega}^{*}=\frac{1}{4 \pi}$, and the single scattering approximation is used. The arrow indicates the part of the distribution that is due to the ballistic photons, i.e., the arrow represents a Dirac distribution. This illustration does not allow for analysis of the ratio of ballistic to scattered photons, but we invite the reader to see Fig. 14. 


\subsubsection{Implementation of the single scattering approximation for an equivalent transport problem: application} to a flat-plate photobioreactor

In the rest of this section, we address the equivalent transport problem defined by $\alpha_{s}^{*}, k_{e x t}^{*}$, and $p_{\Omega}\left(\boldsymbol{\omega} \mid \boldsymbol{\omega}^{\prime}\right)=\frac{1}{4 \pi}$ (see Section 3.2), and we use only scattering orders 0 and 1 (see Eq. 45). Under these conditions, Eq. 49 becomes

$$
L^{(0)}(z, \boldsymbol{\omega})=\frac{q_{\cap}}{\mu_{i}} \exp \left(-k_{e x t}^{*} \frac{z}{\mu_{i}}\right) \delta\left(\boldsymbol{\omega}-\boldsymbol{\omega}_{i}\right)
$$

and substituting this solution into Eq. 51, we obtain the following source term for population (1):

$$
\mathcal{C}^{(0)}(z, \omega)=\frac{\alpha_{s}^{*} k_{e x t}^{*}}{4 \pi} \frac{q_{\cap}}{\mu_{i}} \exp \left(-k_{e x t}^{*} \frac{z}{\mu_{i}}\right)
$$

Due to isotropy of the phase function, the source term $\mathcal{C}^{(0)}(z, \boldsymbol{\omega})$ is independent of the direction $\boldsymbol{\omega}\left(\mathcal{C}^{(0)}\right.$ is isotropic). A solution for $L^{(1)}$ is obtained by solving Eq. 50 under the boundary conditions in Eqs. 52 and 53 . This task can be accomplished either by the variation of constants method or by intuitive reasoning: the intensity $L^{(1)}\left(\mathbf{x}_{0}, \boldsymbol{\omega}_{0}\right)$ is the source term $\mathcal{C}^{(0)}\left(\mathbf{x}_{1}, \boldsymbol{\omega}_{0}\right)$ attenuated by extinction along the length $\left\|\mathbf{x}_{0}-\mathbf{x}_{1}\right\|$, which is integrated over the locations $\mathbf{x}_{1}$ defined by $\mathbf{x}_{1}=\mathbf{x}_{0}-s \boldsymbol{\omega}_{0}$ with $s \in[0,+\infty[$. In Fig. 12, we show that this reasoning indeed involves constructing all the single-scattering optical paths with direction $\boldsymbol{\omega}_{0}$ at $\mathbf{x}_{0}$. In the one-dimensional configuration under study, $L^{(1)}\left(\mathbf{x}_{0}, \boldsymbol{\omega}_{0}\right)$ depends only on the abscissa $z_{0}$ at $\mathbf{x}_{0}$ and on the cosine $\mu_{0}=\boldsymbol{\omega}_{0} \cdot \mathbf{e}_{z}$. In addition, $\left\|\mathbf{x}_{0}-\mathbf{x}_{1}\right\|=\left|\frac{z_{0}-z_{1}}{\mu_{0}}\right|$ (see Fig. 12). For the directions where $\mu_{0}>0$, we obtain

$$
L^{(1)}\left(z_{0}, \boldsymbol{\omega}_{0}\right)=\int_{0}^{z_{0}} \frac{d z_{1}}{\mu_{0}} \mathcal{C}^{(0)}\left(z_{1}\right) \exp \left(-k_{\text {ext }}^{*} \frac{z_{0}-z_{1}}{\mu_{0}}\right)
$$

and for the directions where $\mu_{0}<0$,

$$
L^{(1)}\left(z_{0}, \boldsymbol{\omega}_{0}\right)=\int_{z_{0}}^{E} \frac{d z_{1}}{-\mu_{0}} \mathcal{C}^{(0)}\left(z_{1}\right) \exp \left(-k_{\text {ext }}^{*} \frac{z_{0}-z_{1}}{\mu_{0}}\right)
$$

Substituting Eq. 59 into the above equations and solving the integration, we obtain the following:

- for $\mu_{0}>0$,

$$
L^{(1)}\left(z_{0}, \boldsymbol{\omega}_{0}\right)=\frac{\alpha_{s}^{*}}{4 \pi} \frac{q_{\cap}}{\mu_{0}-\mu_{i}}\left[\exp \left(-k_{e x t}^{*} \frac{z_{0}}{\mu_{0}}\right)-\exp \left(-k_{e x t}^{*} \frac{z_{0}}{\mu_{i}}\right)\right]
$$

- and for $\mu_{0}<0$,

$$
L^{(1)}\left(z_{0}, \boldsymbol{\omega}_{0}\right)=\frac{\alpha_{s}^{*}}{4 \pi} \frac{q_{\cap}}{\mu_{0}-\mu_{i}}\left[\exp \left(-k_{e x t}^{*} \frac{E}{\mu_{i}}\right) \exp \left(-k_{e x t}^{*} \frac{z_{0}-E}{\mu_{0}}\right)-\exp \left(-k_{e x t}^{*} \frac{z_{0}}{\mu_{i}}\right)\right]
$$

Finally, the total intensity $L\left(z_{0}, \boldsymbol{\omega}_{0}\right)$ is estimated as

$$
L\left(z_{0}, \boldsymbol{\omega}_{0}\right) \simeq L^{(0)}\left(z_{0}, \boldsymbol{\omega}_{0}\right)+L^{(1)}\left(z_{0}, \boldsymbol{\omega}_{0}\right)
$$

where $L^{(0)}$ is given in Eq. 58 .

Fig. 13 presents the angular distribution of $L$ resulting from the single-scattering approximation for the equivalent transport problem $\alpha_{s}^{*}, k_{e x t}^{*}$ and $p_{\Omega}^{*}=\frac{1}{4 \pi}$ as well as the reference solution produced by the Monte Carlo method for $\alpha_{s}$ and $k_{e x t}$ and the phase function of $C$. reinhardtii. In the reference situation, at the location in question $\left(z_{0}=3 \mathrm{~cm}\right)$, the ballistic beam is completely attenuated: all the photons have undergone at least one scattering event but deviated very little from their incident direction (see Section 3.2). This situation results in a complex angular distribution centered around the incident direction (see Fig. 13.a). In our equivalent transport problem, this complex distribution is replaced by the sum of a Dirac distribution (contribution of the ballistic photons, i.e., $\sim 75 \%$ of the photons in the present case, see Fig. 14) and 
a relatively broad distribution (contribution of the scattered photons) that is simply modeled as Eqs. 62 and 63 under the single-scattering approximation (see Fig. 13.b). The angular distribution of the scattered intensity $L^{(1)}$ at different locations is shown in Fig. 15.

Integration of $L(z, \boldsymbol{\omega})$ over the directions $\boldsymbol{\omega}$ yields the local irradiance $G(z)$ :

$$
G(z)=\int_{4 \pi} d \boldsymbol{\omega} L(z, \boldsymbol{\omega}) \simeq \int_{4 \pi} d \boldsymbol{\omega} L^{(0)}(z, \boldsymbol{\omega})+\int_{4 \pi} d \boldsymbol{\omega} L^{(1)}(z, \boldsymbol{\omega})=G^{(0)}(z)+G^{(1)}(z)
$$

where $G^{(0)}$ is the irradiance due to the ballistic photons, and $G^{(1)}$ is the irradiance due to the photons that have undergone only one scattering event. The expression $G^{(0)}=\int_{4 \pi} d \boldsymbol{\omega} \frac{q_{\cap}}{\mu_{i}} \exp \left(-k_{\text {ext }}^{*} \frac{z}{\mu_{i}}\right) \delta\left(\boldsymbol{\omega}-\boldsymbol{\omega}_{i}\right)$ is simply

$$
G^{(0)}(z)=\frac{q_{\cap}}{\mu_{i}} \exp \left(-k_{\text {ext }}^{*} \frac{z}{\mu_{i}}\right)
$$

Obtaining $G^{(1)}$ is usually less straightforward, but the integral in Eq. 65 has a symbolic solution in the present case:

$$
\begin{aligned}
& G^{(1)}(z)=\frac{\alpha_{s}^{*}}{2} q_{\cap} \times \\
& \left\{\exp \left(-k_{e x t}^{*} \frac{z}{\mu_{i}}\right)\left(E i\left[k_{e x t}^{*} z \frac{1-\mu_{i}}{\mu_{i}}\right]-E i\left[-k_{e x t}^{*}(E-z) \frac{1+\mu_{i}}{\mu_{i}}\right]+\ln \left[\frac{1+\mu_{i}}{1-\mu_{i}}\right]\right)\right. \\
& \left.\quad-E i\left[-k_{\text {ext }}^{*} z\right]+\exp \left(-k_{\text {ext }}^{*} \frac{E}{\mu_{i}}\right) E i\left[-k_{\text {ext }}^{*}(E-z)\right]\right\}
\end{aligned}
$$

where $E i$ is the exponential integral $E i(x)=-\int_{-x}^{\infty} d t \frac{e^{-t}}{t}$, which is a function available in most scientific computation libraries. In the special case of normal incidence $\mu_{i} \rightarrow 1, G^{(1)}$ becomes

$$
\begin{gathered}
G^{(1)}\left(z ; \mu_{i}=1\right)=\frac{\alpha_{s}^{*}}{2} q_{\cap}\left\{\exp \left(-k_{e x t}^{*} z\right)\left(\gamma+\ln [2]+\ln \left[k_{e x t}^{*} z\right]-E i\left[-2 k_{\text {ext }}^{*}(E-z)\right]\right)\right. \\
\left.-E i\left[-k_{e x t}^{*} z\right]+\exp \left(-k_{e x t}^{*} E\right) E i\left[-k_{\text {ext }}^{*}(E-z)\right]\right\}
\end{gathered}
$$

where $\gamma \simeq 0.577$ is the Euler-Mascheroni constant.

The irradiance field obtained with the single-scattering approximation is shown in Fig. 14. Panel a shows the proportion of ballistic and scattered photons within the reaction volume. As indicated by the intermediate value of the transport optical thickness $e_{s}^{*}=1.1$, the scattered photons are in the minority but cannot be disregarded. Fig. 14.b shows comparison between the reference solution (Monte Carlo method) and the results obtained by combining the equivalent transport problem and the single-scattering approximation. These results indicate that indeed, scattering orders higher that 1 can be ignored during analysis the equivalent problem. Given the agreement observed, we should note that the simple physical interpretations that we developed here are relevant to analysis of the process. In particular, substitution of the complex distribution observed in Fig. 13 by the sum of a Dirac distribution and a wider distribution is extremely convenient. With this approach, description of the ballistic photons is straightforward, and all difficulty of the analysis is reduced to description of the scattered photons. Because the scattered intensity is relatively close to isotropic (see Fig. 15), we can derive the relevant macroscopic description of the scattered photons in the next section. 

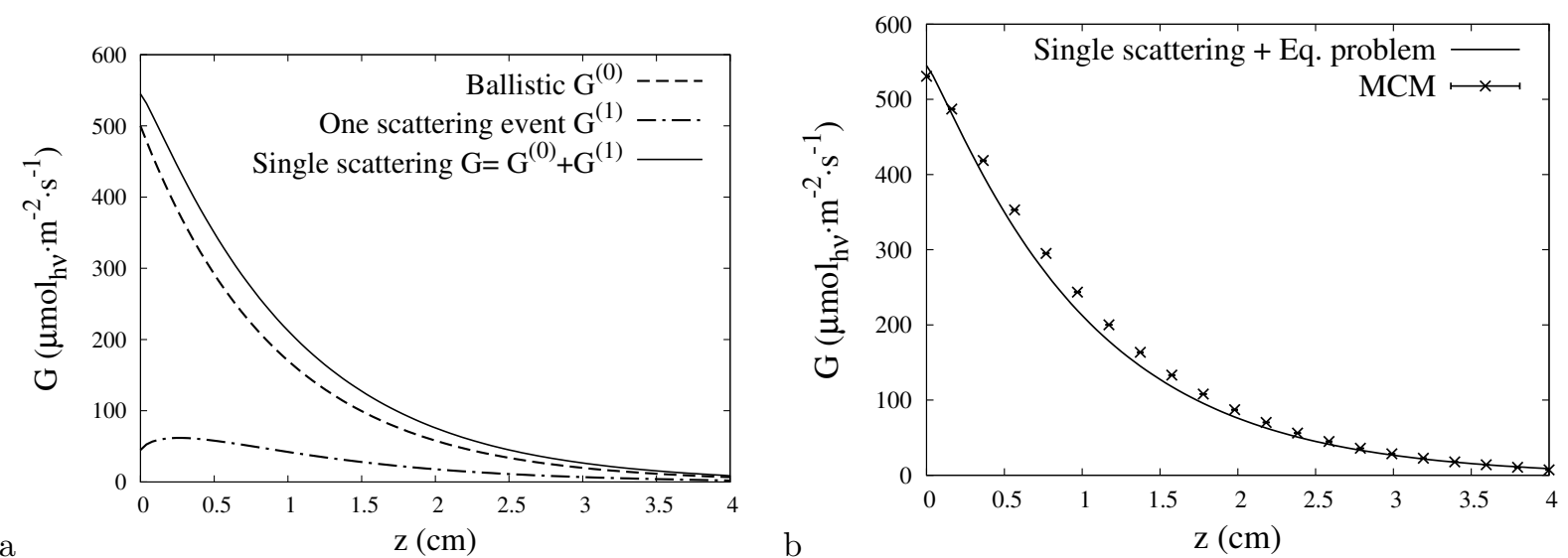

Figure 14: The irradiance field $G$ within the photobioreactor shown in Fig. $6 ; \rho^{\mathcal{F}}=\rho^{\mathcal{R}}=0$ and collimated normal incidence $\mu_{i}=1$. The results were obtained for the equivalent transport problem where $\alpha_{s}^{*}=0.25, k_{\text {ext }}^{*}=110 \mathrm{~m}^{-1}$, and $p_{\boldsymbol{\Omega}}^{*}=1 / 4 \pi$ : the expression for $G^{(0)}$ is given in Eq. 66, the expression for $G^{(1)}$ is given in Eq. 68 (note that Eq. 67 must be used instead if $\left.\mu_{i} \neq 1\right)$. The single-scattering approximation is used: $G=G^{(0)}+G^{(1)}$. a: The proportions of ballistic and scattered photons. $\mathrm{b}$ : Comparison with the reference solution obtained by the Monte Carlo method (MCM) for $\alpha_{s}=0.86, k_{e x t}=587 \mathrm{~m}^{-1}$, and the phase function of Chlamydomonas reinhardtii (see Section 4).

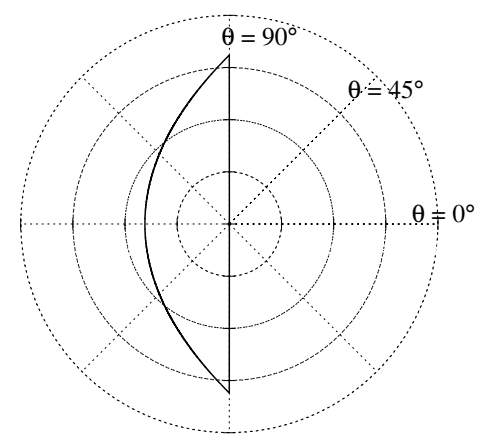

a

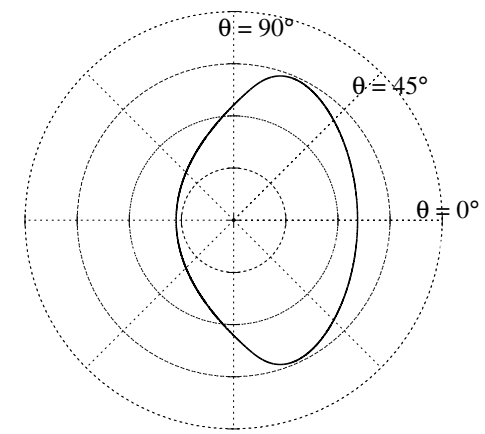

d

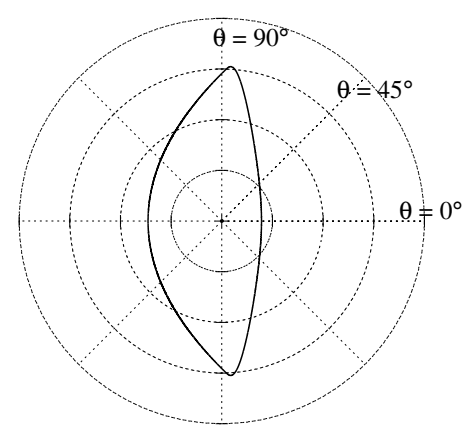

$\mathrm{b}$

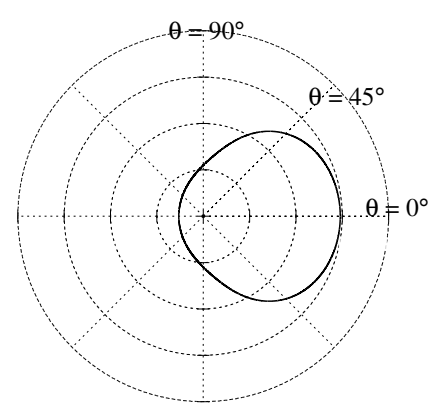

$\mathrm{c}$

$\mathrm{f}$
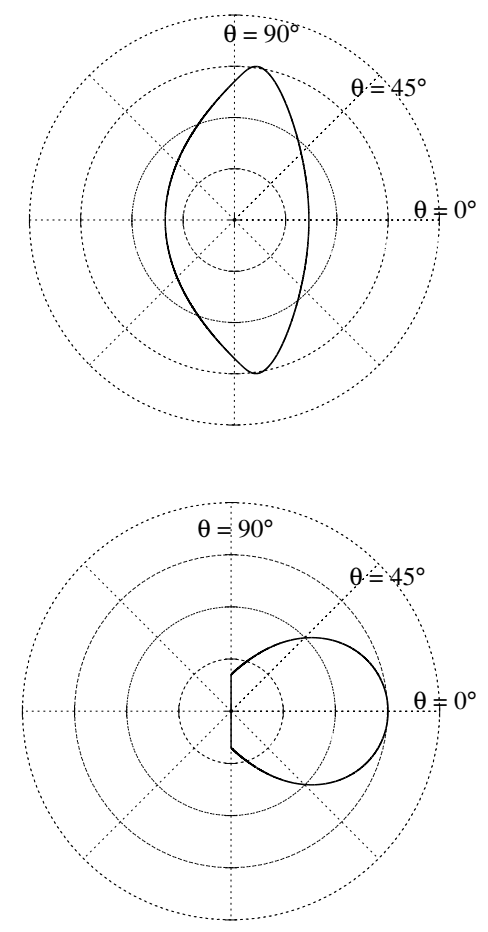

Figure 15: Angular distribution of the scattered intensity $L^{(1)}\left(z_{0}, \theta\right)$ at the abscissa $z_{0}$ within the photobioreactor shown in Fig. $6 ; \rho^{\mathcal{F}}=\rho^{\mathcal{R}}=0$; collimated normal incidence $\mu_{i}=1$. The results were obtained for the equivalent transport problem where $\alpha_{s}^{*}=0.25, k_{\text {ext }}^{*}=110 \mathrm{~m}^{-1}$, and $p_{\Omega}^{*}=1 / 4 \pi$, according to Eqs. 62 and 63 . a: $z_{0}=0$. b: $z_{0}=2.5 \mathrm{~mm}$. c: $z_{0}=5 \mathrm{~mm}$. d: $z_{0}=1 \mathrm{~cm}$. e: $z_{0}=2.5 \mathrm{~cm}$. f: $z_{0}=4 \mathrm{~cm}$ 


\subsection{The P1 approximation and diffusion equation}

In contrast to the single-scattering approximation (previous paragraph), which is mesoscopic, below we adopt a macroscopic point of view. In this section and in Section 3.5, angular distribution of intensity is fixed a priori, and the radiative transfer equation is integrated over all propagation directions in order to formulate a closed equation for the irradiance.

\subsubsection{The diffusion equation in one-dimensional Cartesian geometric configuration}

The P1 approximation consists of truncating the spherical-harmonic expansion of the intensity at order 1. In the one-dimensional configuration shown in Fig. 6, for Lambertian or collimated normal incidence, this method is equivalent to fixing the following functional form for the angular dependence:

$$
L(\mathbf{x}, \boldsymbol{\omega})=A(z)[1+C(z) \cos (\theta)]
$$

where, given the symmetry of the problem, $L$ is a function of the abscissa $z$ and the angle $\theta$ only $(\cos (\theta)=$ $\boldsymbol{\omega} \cdot \mathbf{e}_{z}$ ). Substituting this approximation into the radiative transfer equation Eq. 24 (in which we omit the frequency variable) and integrating it over all the propagation directions $\boldsymbol{\omega}$ (i.e., across all angles $\theta$ ), we obtain a diffusion equation for the description of the irradiance field [66]:

$$
-\tilde{D} \partial_{z}^{2} G(z)=-c k_{a} G(z)
$$

where $\partial_{z}^{2} G(z)$ is the second derivative of the irradiance with respect to $z$, and $\tilde{D}$ is the macroscopic diffusion coefficient: $\tilde{D}=c /\left(3 k_{\text {ext }}\left(1-\alpha_{s} g\right)\right)=c /\left(3 k_{\text {ext }}^{*}\right) ; k_{\text {ext }}^{*}$ is defined in Section 3.2. Hereafter, we will express the diffusion coefficient in $m$ rather than in $\mathrm{m}^{2} / \mathrm{s}$; this approach is convenient for analysis of steady-state systems. Indeed, in this case, the solution of the radiative transfer equation is independent of the speed of light $c$; accordingly, it is customary to divide Eq. 70 by $c$ :

$$
-D \partial_{z}^{2} G(z)=-k_{a} G(z)
$$

with the macroscopic diffusion coefficient $D$ expressed in $m$, defined as $D=\tilde{D} / c$ :

$$
D=\frac{1}{3 k_{e x t}\left(1-\alpha_{s} g\right)}=\frac{1}{3 k_{e x t}^{*}}
$$

It should be noted that Fick's equation is also obtained by substituting Eq. 69 into the radiative transfer equation, multiplying it by $\cos (\theta)$, and integrating over the propagation directions [66]:

$$
q(z)=-D \partial_{z} G(z)
$$

where $q(z)$ is the surface flux density along $\mathbf{e}_{z}$ (see Eqs. 33 and 34 ).

Boundary conditions. The steady-state diffusion equation (Eq. 71) is an ordinary differential equation of order 2, whose solution requires two boundary conditions. In radiative transfer, the value of the irradiance or the net flux at the boundary is rarely available. Therefore, a linear relation between $G$ and its derivative, that is, between the irradiance and the flux (see Eq. 73) is generally used for the boundary conditions: this is what researchers in this field call the Marshak boundary conditions [67]. To our knowledge, in the existing literature, the expression for Marshak's boundary conditions is brought to the following functional form $[62,66,68]$ :

$$
\left[G \pm \mathcal{L} \partial_{s} G\right]=\mathcal{B}
$$

where $\mathcal{L}$ is the extrapolation length, and $\mathcal{B}$ is a constant. Determination of parameters $\mathcal{L}$ and $\mathcal{B}$ that ensure the same order of approximation near the boundaries as within the medium is a topic of research in itself (e.g., see [68]). In the context of photobioreactor analysis, we use a specific expression for $\mathcal{B}$ (derived in [69]), which leads to the following boundary conditions in the case of the flat-plate photobioreactor shown in Fig. 6: 
- At $z=0$,

$$
G(0)-\mathcal{L}_{0} \partial_{z} G(0)=2 \frac{1+\rho^{\mathcal{F}}}{1-\rho^{\mathcal{F}}} q^{(0)}(0)+G^{(0)}(0)
$$

- At $z=E$,

$$
G(E)+\mathcal{L}_{E} \partial_{z} G(E)=2 \frac{1+\rho^{\mathcal{R}}}{1-\rho^{\mathcal{R}}} q^{(0)}(E)+G^{(0)}(E)
$$

where the exponent ${ }^{(0)}$ deals with the ballistic photons (see Section 3.3), and $\mathcal{L}$ is the extrapolation length that is estimated here as in [68]:

$$
\begin{aligned}
& \mathcal{L}_{0}=\frac{2}{3} \frac{1+\rho^{\mathcal{F}}}{1-\rho^{\mathcal{F}}} \frac{1}{k_{\text {ext }}^{*}} \\
& \mathcal{L}_{E}=\frac{2}{3} \frac{1+\rho^{\mathcal{R}}}{1-\rho^{\mathcal{R}}} \frac{1}{k_{\text {ext }}^{*}}
\end{aligned}
$$

where $\rho$ is reflectivity of the bounding surface, and $k_{e x t}^{*}$ is defined in Eq. 40. $q^{(0)}$ and $G^{(0)}$ are respectively the surface flux density and the irradiance corresponding to the ballistic photons emitted at the boundary. The analytical solution of the diffusion equation for these boundary conditions in the case of Lambertian illumination is derived in the following section.

\subsubsection{The case of diffuse illumination: direct solution of the diffusion equation}

In the text below, we will solve the diffusion equation (Eq. 71) for the boundary conditions (Eqs. 75 and 76) for the case of the one-dimensional configuration shown in Fig. 6 with Lambertian emission at $z=0$ and reflection at $z=E$. The general solution that satisfies Eq. 71 is

$$
G(z)=C_{0} \exp (\xi z)+C_{1} \exp (-\xi z)
$$

where $C_{0}$ and $C_{1}$ are constants, and $\xi=\sqrt{k_{a} / D}$ (in the configuration under study $\xi \simeq 161$ ). The boundary condition at $z=0$ is (according to Eqs. 75 and 77 where $\rho^{\mathcal{F}}=0$ ):

$$
G(0)-\mathcal{L}_{0} \partial_{z} G(0)=2 q^{(0)}(0)+G^{(0)}(0)
$$

with

$$
\mathcal{L}_{0}=\frac{2 / 3}{k_{\text {ext }}^{*}}
$$

We will now focus on the expression for $G^{(0)}(0)$ and $q^{(0)}(0)$, which are ballistic irradiance and ballistic surface flux density, respectively (see Section 3.3). The mesoscopic definition of Lambertian emission (according to Eq. 26) results in

$$
L^{(0)}(0, \boldsymbol{\omega})=q_{\cap} / \pi \text { for } \theta \in[0, \pi / 2]
$$

where $q_{\cap}$ is the incident surface flux density. Moreover, in the present case, we assume that $L^{(0)}(0, \boldsymbol{\omega})=0$ for $\theta \in[\pi / 2, \pi]$ because the ballistic optical paths reflected at $z=E$ are completely attenuated when they return at $z=0$ (the scattering optical thickness is $e_{s} \simeq 20$ ). Therefore, the intensity is integrated easily, according to Eqs. 30 and 33:

$$
\begin{gathered}
G^{(0)}(0)=\int_{4 \pi} L^{(0)}(0, \boldsymbol{\omega}) d \boldsymbol{\omega}=2 q_{\cap} \\
q^{(0)}(0)=\int_{4 \pi} L^{(0)}(0, \boldsymbol{\omega}) \boldsymbol{\omega} \cdot \mathbf{e}_{z} d \boldsymbol{\omega}=q_{\cap}
\end{gathered}
$$

Hence

$$
G(0)-\mathcal{L}_{0} \partial_{z} G(0)=4 q_{\cap}
$$


For the boundary condition at $z=E$, ballistic photons can be ignored (they are scattered or absorbed before reaching $z=E$, as in the case of the boundary conditions at $z=0$ above ${ }^{6}$ ), i.e., $q^{(0)} \simeq 0$ and $G^{(0)} \simeq 0$. Therefore, Eq. 76 becomes

$$
G(E)+\mathcal{L}_{E} \partial_{z} G(E)=0
$$

Constants $C_{0}$ and $C_{1}$ in the general solution (Eq. 78) that satisfy the boundary conditions (Eqs. 84 and 85 ) are

$$
C_{1}=\frac{4 q_{\cap}}{\exp (-\xi 2 E) \frac{\mathcal{L}_{E} \xi-1}{\mathcal{L}_{E} \xi+1}\left(1-\mathcal{L}_{0} \xi\right)+1+\mathcal{L}_{0} \xi}
$$

and

$$
C_{0}=C_{1} \frac{\mathcal{L}_{E} \xi-1}{\mathcal{L}_{E} \xi+1} \exp (-\xi 2 E)
$$

Thus, we obtain the following expression for the irradiance field:

$$
G(z)=4 q_{\cap} C\left(\exp [-\xi z]+\frac{\mathcal{L}_{E} \xi-1}{\mathcal{L}_{E} \xi+1} \exp [-\xi(2 E-z)]\right)
$$

where

$$
C=\frac{1}{\exp (-\xi 2 E) \frac{\mathcal{L}_{E} \xi-1}{\mathcal{L}_{E} \xi+1}\left(1-\mathcal{L}_{0} \xi\right)+1+\mathcal{L}_{0} \xi}
$$

From the mesoscopic point of view, the P1 approximation (according to Eq. 69) gives us

$$
L(z, \boldsymbol{\omega})=\frac{1}{4 \pi}\left[G(z)-D \partial_{z} G(z) \cos (\theta)\right]
$$

where

$$
\partial_{z} G(z)=4 q_{\cap} C \xi\left(-\exp [-\xi z]+\frac{\mathcal{L}_{E} \xi-1}{\mathcal{L}_{E} \xi+1} \exp [-\xi(2 E-z)]\right)
$$

Figs. 16 and 17 represent respectively the irradiance field and the angular distribution of the intensity ${ }^{7}$ obtained with the P1 approximation. Although the situation under study is far from equilibrium, the irradiance field produced by the approximation is in good agreement with the reference solution. This correspondence is surprising because P1 should be unsuitable for such a situation with intermediate optical thickness. The next paragraph is focused on the validity conditions of the P1 approximation.

\footnotetext{
${ }^{6}$ Contrary to Section 3.3, where we addressed the equivalent transport problem, ballistic photons here are in the minority, except close to $z=0$, for $\theta \in[0, \pi / 2]$. It is possible to take into account all the ballistic photons in our calculations (Eqs. 75 and 76) because the mesoscopic solution for $L^{(0)}$ is obtained easily, even in the present case, with Lambertian emission and reflection at $z=E$. Nonetheless, except for the term that we used in Eq. 84, their contribution to the boundary conditions is negligible for most photobioreactor configurations during operation close to the optimum biomass growth rate.

${ }^{7}$ In the angular distributions presented in Fig. 17, we use the same scale for the P1 approximation and the Monte Carlo method. Note that the area under the curve does not represent the irradiance because the element of $\operatorname{solid}$ angle $\sin (\theta) d \theta d \varphi$ is not taken into consideration here: $G=2 \pi \int_{0}^{\pi} d \theta \sin (\theta) L(\theta)$.
} 


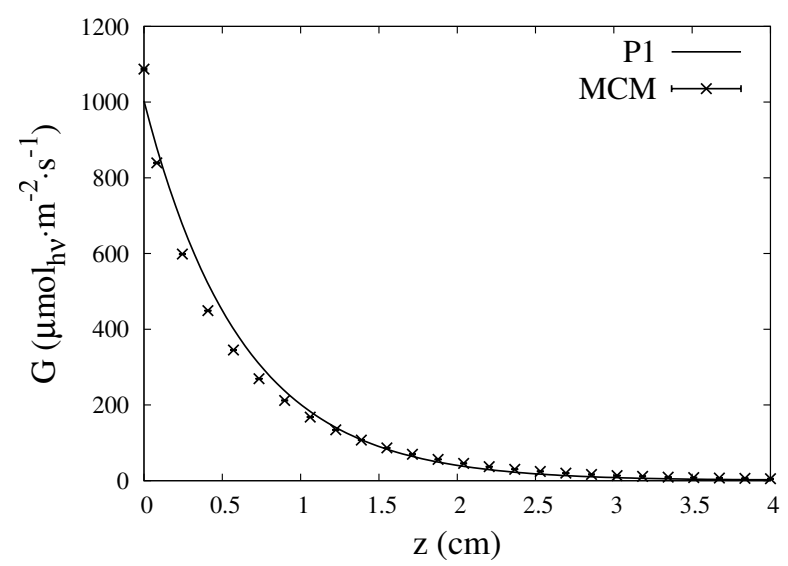

Figure 16: The irradiance field $G$ within the photobioreactor shown in Fig. $6 ; \rho^{\mathcal{F}}=0, \rho^{\mathcal{R}}=0.54$, and Lambertian incidence. Comparison between the P1 approximation (Eq. 88) and the reference solution (Monte Carlo method, MCM).
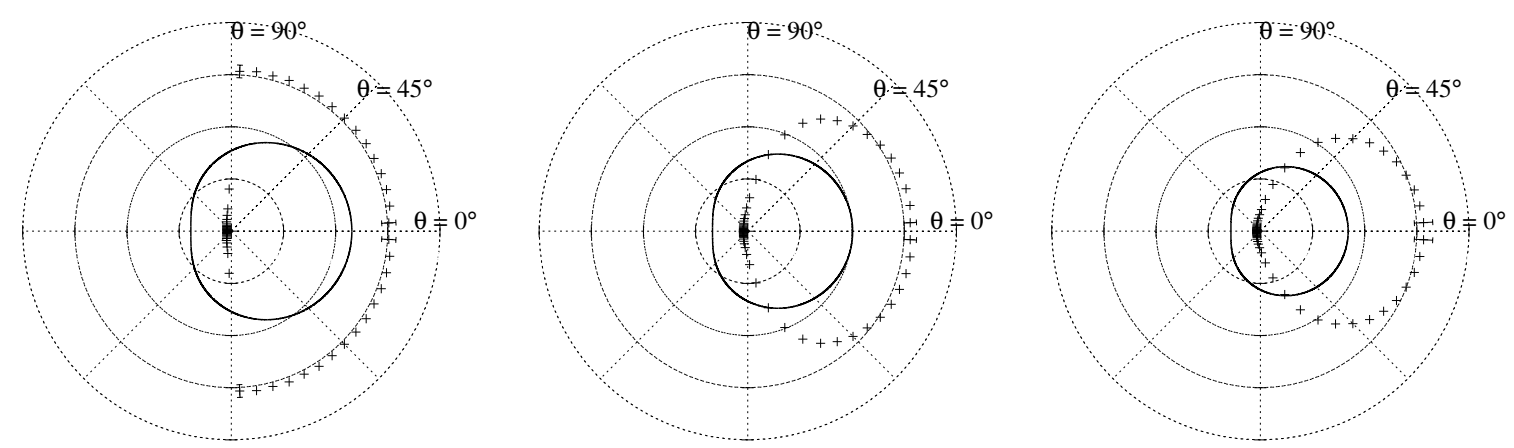

a

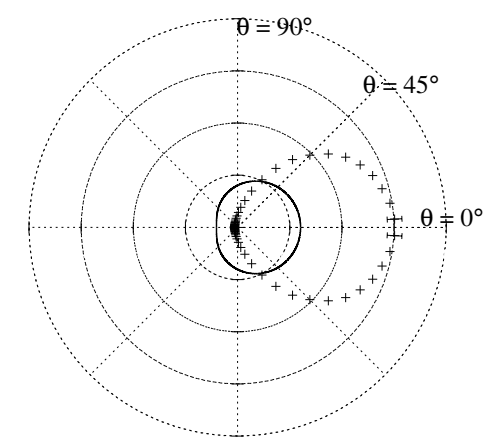

$\mathrm{d}$

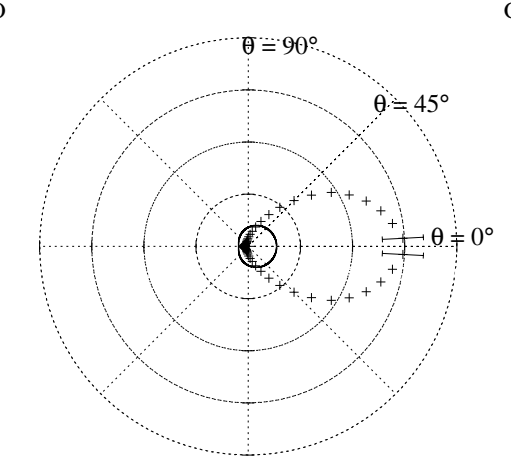

e

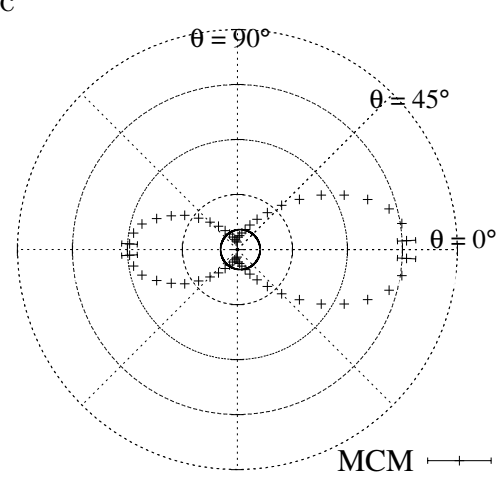

f

Figure 17: Angular distribution of the intensity $L\left(z_{0}, \theta\right)$ at the abscissa $z_{0}$ within the photobioreactor shown in Fig. 6 ; $\rho^{\mathcal{F}}=0$, $\rho^{\mathcal{R}}=0.54$, and Lambertian incidence. Comparison between the P1 approximation (Eq. 90) and the reference solution (Monte Carlo method, MCM). a: $z_{0}=0$. b: $z_{0}=2.5 \mathrm{~mm}$. c: $z_{0}=5 \mathrm{~mm}$. d: $z_{0}=1 \mathrm{~cm}$. e: $z_{0}=2.5 \mathrm{~cm}$. f: $z_{0}=4 \mathrm{~cm}$. 


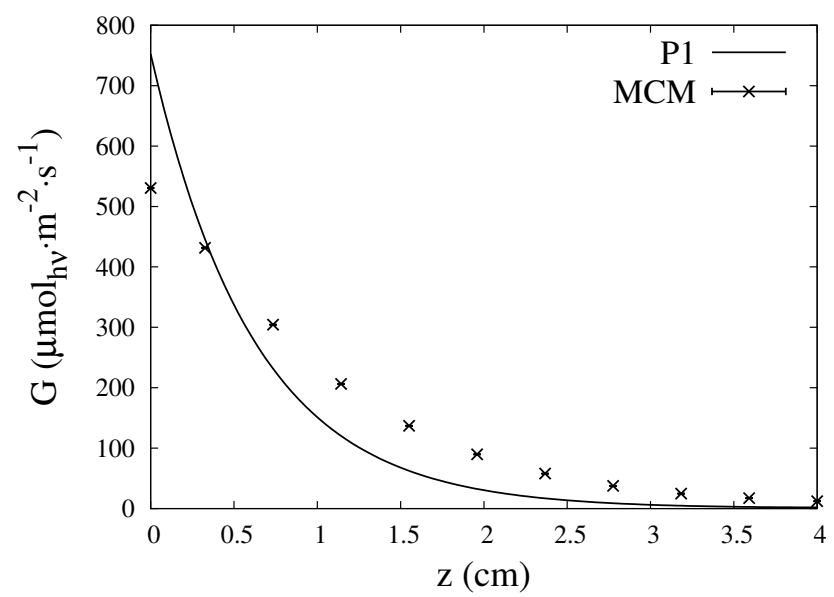

Figure 18: The irradiance field $G$ within the photobioreactor shown in Fig. 6, with the same parameters as in Fig. 16, but the Lambertian emission is replaced by collimated emission at $\theta_{i}=0$. Comparison between the P1 approximation (Eq. 88) and the reference solution (Monte Carlo method, MCM). For collimated incidence, only the boundary condition at $z=0$ is modified, in comparison with the solution used in Fig. 16. We still have $q^{(0)}(z=0)=q_{\cap}$, but the ballistic irradiance becomes $G^{(0)}(z=0)=q_{\cap} / \mu_{i}$. Therefore, the same solution as in Fig. 16 can be used, but with replacement of $4 q_{\cap}$ with $\left(2+1 / \mu_{i}\right) q_{\cap}$ in Eq. 88.

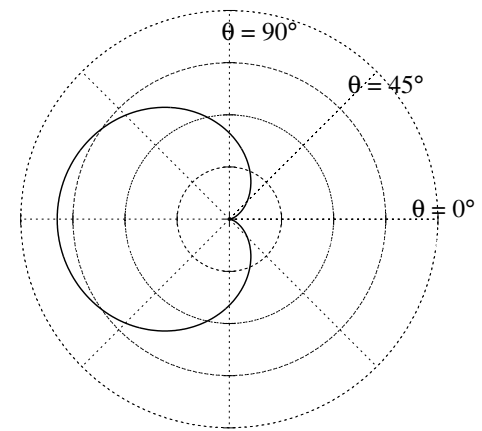

a

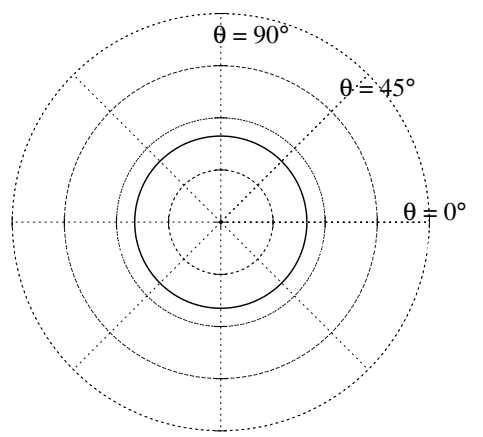

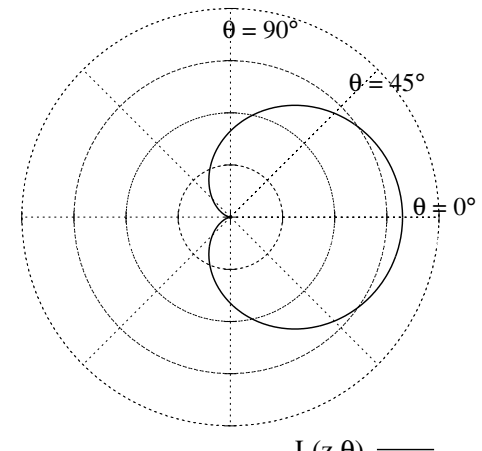

$\mathrm{L}(\mathrm{z}, \theta)$

Figure 19: Angular distribution of the intensity for the P1 approximation: $L(z, \theta)=A(z)[1+C(z) \cos (\theta)]$, where $C(z) \in$ $[-1,1]$. a: $C(z)=-1$; b: isotropic distribution $C(z)=0$; : $C(z)=1$.

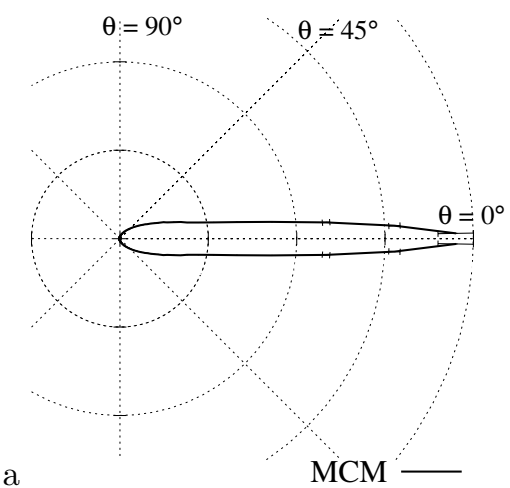

a

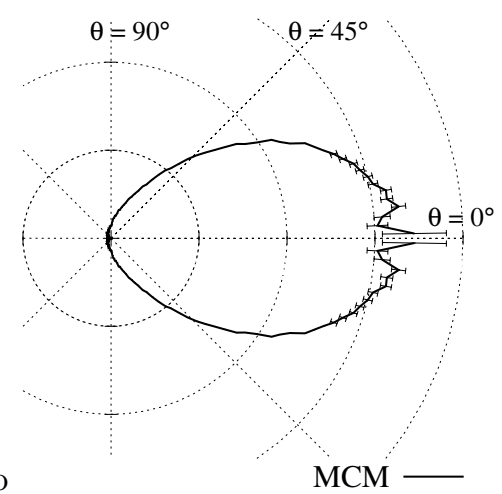

Figure 20: Angular distribution of the intensity $L(z, \theta)$ within the photobioreactor shown in Fig. 6 . The results were obtained by the Monte Carlo method, a: for collimated normal incidence, b: for Lambertian incidence (diffuse illumination). 


\subsubsection{Validity conditions of the P1 approximation}

These conditions are frequently defined as

$$
e_{s}^{*} \gg 1
$$

where $e_{s}^{*}$ is the scattering optical thickness defined in Eq. 43. In the situation studied in Fig. 16, $e_{s}^{*}=1.1$, and the approximation already works well. If we now address the same situation but replace the Lambertian illumination with a collimated source (the situation corresponding to Fig. 18), then the approximation does not work at all. In these two configurations, optical thickness has the same value, and yet the P1 approximation works well in one case but not in the other. In the text below, we explore the validity conditions of the P1 approximation, and the results will lead to a strategy for analysis of collimated illumination.

The P1 approximation postulates the functional form

$$
L(\mathbf{x}, \boldsymbol{\omega})=A(z)[1+C(z) \cos (\theta)]
$$

for the intensity $L$ (i.e., Eq. 69). It is therefore valid if the intensity can be represented by this functional form: this is the only strict definition that can be formulated for validity of the P1 approximation. This form corresponds to situations where the intensity is close to isotropy (i.e., near-equilibrium situations ${ }^{8}$ ). In fact, in the above equation, $C(z) \in[-1,1]$ because otherwise the intensity may be negative. Thus, we see in Fig. 19 that the range of angular distributions resulting from the P1 approximation is not compatible with the description of a photobioreactor during collimated illumination (see Fig. 20.a). The figure shows the angular distribution of the intensity as a function of the boundary conditions: for Lambertian emission, light enters the medium from all directions, resulting in intensity that is much closer to isotropy, in comparison with collimated incidence. We thus understand that the P1 approximation allows us to analyze Lambertian emission (Section 3.4.2), even in a situation far from equilibrium. We also see that this approximation fails in the case of collimated incidence in Fig. 18. The condition $e_{s}^{*} \gg 1$ (mentioned above) usually ensures that the intensity is close to isotropy (because of a large number of isotropic scattering events), but the P1 approximation is actually less restrictive: it is sufficient that the intensity is compatible with Eq. 69, regardless of the scattering phenomenon. This situation will allow us to develop an approach to analysis of collimated illumination phenomena in the paragraphs that follow.

\subsubsection{The case of collimated illumination: separation of ballistic and diffusive contributions}

Separation between ballistic and scattered photons. In Section 3.3, Fig. 13, we saw that the equivalent transport problem allows us to separate our radiative study into two simple systems: the ballistic photons, for which the exact solution is analytical, and the scattered photons, which correspond to intensity close to isotropy. This relative isotropy of the scattered intensity in the equivalent transport problem suggests that the P1 approximation is relevant. Therefore, to formulate the collimated incidence phenomena, we will address the equivalent transport problem and separate the analysis of ballistic photons from that of scattered photons: only scattered photons will be subjected to the P1 approximation. In the rest of the chapter, the ballistic population is denoted as $(0)$, whereas the scattered photons will be called "the diffuse population" and denoted as $(d)$.

It is always possible to formulate the irradiance of the entire photon population as the sum of ballistic irradiance and diffuse irradiance (as is the case for the intensity in Eq. 44):

$$
G(z)=G^{(0)}(z)+G^{(d)}(z)
$$

where $G^{(d)}$ is defined as the sum of the irradiance values for all scattering orders $j \geq 1, G^{(d)}(z)=$ $\sum_{j=1}^{\infty} G^{(j)}(z)$ (see Section 3.3.1). The rigorous solution for the ballistic irradiance $G^{(0)}$ is easy to obtain: we already did so in the context of the single-scattering approximation, in Eq. 66. The diffuse irradiance

\footnotetext{
${ }^{8}$ We will remind readers that here, the angular distribution of the intensity in question is completely different from the angular distribution of the phase function most of the time.
} 
$G^{(d)}$ is the solution to a radiative transfer problem in which the source $S_{G^{(d)}}$ is isotropic and distributed throughout all the reaction volume: this source represents ballistic photons that are scattered for the first time in the medium ${ }^{9}$, exactly as in Section 3.3. Therefore, the diffusion equation for $G^{(d)}$ is the same as in Eq. 71 but with addition of the source term $S_{G^{(d)}}$ :

$$
-D \partial_{z}^{2} G^{(d)}(z)=-k_{a} G^{(d)}(z)+S_{G^{(d)}}(z)
$$

where the macroscopic diffusion coefficient $D$ and the absorption coefficient $k_{a}$ are identical to those used in Section 3.4.2 for the analysis of Lambertian emission. This is because they are invariant with the transformation Eqs. 72 and 40.

In the text below, we address the equivalent transport problem defined by $\alpha_{s}^{*}, k_{\text {ext }}^{*}$, and $p_{\Omega}^{*}\left(\boldsymbol{\omega} \mid \boldsymbol{\omega}^{\prime}\right)=\frac{1}{4 \pi}$ (see Section 3.2), and we analyze the one-dimensional configuration shown in Fig. 6 with collimated incidence at $z=0$ and a non-reflecting surface at $z=E$. We use the solution obtained in Section 3.3 for ballistic irradiance (according to Eq. 66):

$$
G^{(0)}(z)=\frac{q_{\cap}}{\mu_{i}} \exp \left(-k_{e x t}^{*} \frac{z}{\mu_{i}}\right)
$$

where $\mu_{i}=\cos \left(\theta_{i}\right)$ is the cosine of the angle of incidence. Then, we address the diffuse irradiance $G^{(d)}$ by solving the diffusion equation (Eq. 93) where the source term is

$$
S_{G^{(d)}}(z)=\int_{4 \pi} d \omega \mathcal{C}^{(0)}(z)=4 \pi \mathcal{C}^{(0)}(z)=\alpha_{s}^{*} k_{\text {ext }}^{*} \frac{q_{\cap}}{\mu_{i}} \exp \left(-k_{\text {ext }}^{*} \frac{z}{\mu_{i}}\right)
$$

where $\mathcal{C}^{(0)}(z)$ is the mesoscopic source term discussed in Section 3.3 (see Eq. 59). Compared to Section 3.4.2, here, we replaced the sources at the boundaries (responsible for the strong anisotropy of the intensity) by an isotropic source distributed throughout the entire volume. The general solution that satisfies the diffusion equation (Eq. 93) is

$$
G^{(d)}(z)=\frac{q_{\cap}}{\mu_{i}}\left[C_{0} \exp (\xi z)+C_{1} \exp (-\xi z)-\frac{\xi^{2}-k_{\text {ext }}^{*} / D}{\xi^{2}-\left(k_{\text {ext }}^{*} / \mu_{i}\right)^{2}}\left(\exp \left[-k_{\text {ext }}^{*} \frac{z}{\mu_{i}}\right]-\exp [-\xi z]\right)\right]
$$

where $C_{0}$ and $C_{1}$ are constants, and $\xi=\sqrt{k_{a} / D}$ (in the configuration under study $\xi \simeq 161$ ).

Boundary conditions. Here, the ballistic photons are analyzed separately; therefore, $G^{(0)}=0$ and $q^{(0)}=0$ in Eqs. 75 and 76 (there is no source at the boundary for the diffuse population). In addition, we ignore reflectivity (i.e., $\rho=0$ ); thus, we have the boundary conditions as follows:

- At $z=0$,

$$
G^{(d)}(0)-\mathcal{L}_{0} \partial_{z} G^{(d)}(0)=0
$$

- At $z=E$,

$$
G^{(d)}(E)+\mathcal{L}_{E} \partial_{z} G^{(d)}(E)=0
$$

where

$$
\mathcal{L}_{0}=\mathcal{L}_{E}=\frac{2 / 3}{k_{\text {ext }}^{*}}
$$

Constants $C_{0}$ and $C_{1}$ in Eq. 96 that satisfy the boundary conditions (Eqs. 97 and 98 ) are

$$
\begin{aligned}
& C_{1}=-\frac{\xi^{2}-k_{\text {ext }}^{*} / D}{\xi^{2}-\left(k_{\text {ext }}^{*} / \mu_{i}\right)^{2}} \times \\
& \frac{\mathcal{L}_{0}\left(k_{\text {ext }}^{*} / \mu_{i}-\xi\right) \frac{1+\mathcal{L}_{E} \xi}{1-\mathcal{L}_{0} \xi}+\exp [-\xi 2 E]\left\{1-\mathcal{L}_{E} \xi-\left(1-\frac{\mathcal{L}_{E} k_{\text {ext }}^{*}}{\mu_{i}}\right) \exp \left[\left(\xi-k_{\text {ext }}^{*} / \mu_{i}\right) E\right]\right\}}{\exp [-\xi 2 E]\left(1-\mathcal{L}_{E} \xi\right)-\left(1+\mathcal{L}_{0} \xi\right) \frac{1+\mathcal{L}_{E} \xi}{1-\mathcal{L}_{0} \xi}}
\end{aligned}
$$

\footnotetext{
${ }^{9}$ These scattering events are isotropic because the phase function is isotropic in the equivalent transport problem as defined in Section 3.2.
} 
and

$$
C_{0}=-C_{1} \frac{1+\mathcal{L}_{0} \xi}{1-\mathcal{L}_{0} \xi}-\mathcal{L}_{0} \frac{\xi^{2}-k_{e x t}^{*} / D}{\left(\xi+k_{e x t}^{*} / \mu_{i}\right)\left(1-\mathcal{L}_{0} \xi\right)}
$$

Finally, the irradiance $G(z)$ is obtained by adding up the ballistic irradiance (Eq. 94) and the diffuse irradiance (Eq. 96):

$$
\begin{aligned}
G(z)=\frac{q_{\cap}}{\mu_{i}}\left[\exp \left(-k_{e x t}^{*} \frac{z}{\mu_{i}}\right)+C_{0} \exp (\xi z)+C_{1} \exp (-\xi z)\right. \\
\left.\quad-\frac{\xi^{2}-k_{e x t}^{*} / D}{\xi^{2}-\left(k_{e x t}^{*} / \mu_{i}\right)^{2}}\left(\exp \left[-k_{\text {ext }}^{*} \frac{z}{\mu_{i}}\right]-\exp [-\xi z]\right)\right]
\end{aligned}
$$

From the mesoscopic point of view, the P1 approximation yields the following diffuse intensity:

$$
L^{(d)}(z, \boldsymbol{\omega})=\frac{1}{4 \pi}\left[G^{(d)}(z)-D \partial_{z} G^{(d)}(z) \cos (\theta)\right]
$$

The total intensity $L$ is the sum of $L^{(d)}$ and of the contribution of ballistic photons, that is, a Dirac distribution centered at the incident direction.

Figs. 21 and 22 show respectively the irradiance field and the angular distribution of the diffuse intensity obtained with the P1 approximation of the equivalent transport problem. Fig. 21.b shows comparison with the reference solution. As we expected in the previous paragraph, the agreement here is significantly improved in comparison with Fig. 18. On the one hand, the solution for the ballistic irradiance is exact; on the other hand, the description of the diffuse population is now compatible with restrictions of the P1 approximation. The angular distributions of the diffuse intensity are compared with the results obtained for the single-scattering approximation in Fig. 15. We show this comparison because these are the two solutions that we obtained for the equivalent transport problem, but these formulae cannot serve as a reference solution. We see, however, that the single-scattering approximation is more likely to describe the phenomena at the boundary because it takes into account the discontinuity phenomena of the intensity angular distribution, whereas the P1 approximation requires spherical-harmonic expansion. Inside the reaction volume, the two approximations yield the diffuse intensity close to isotropy. 

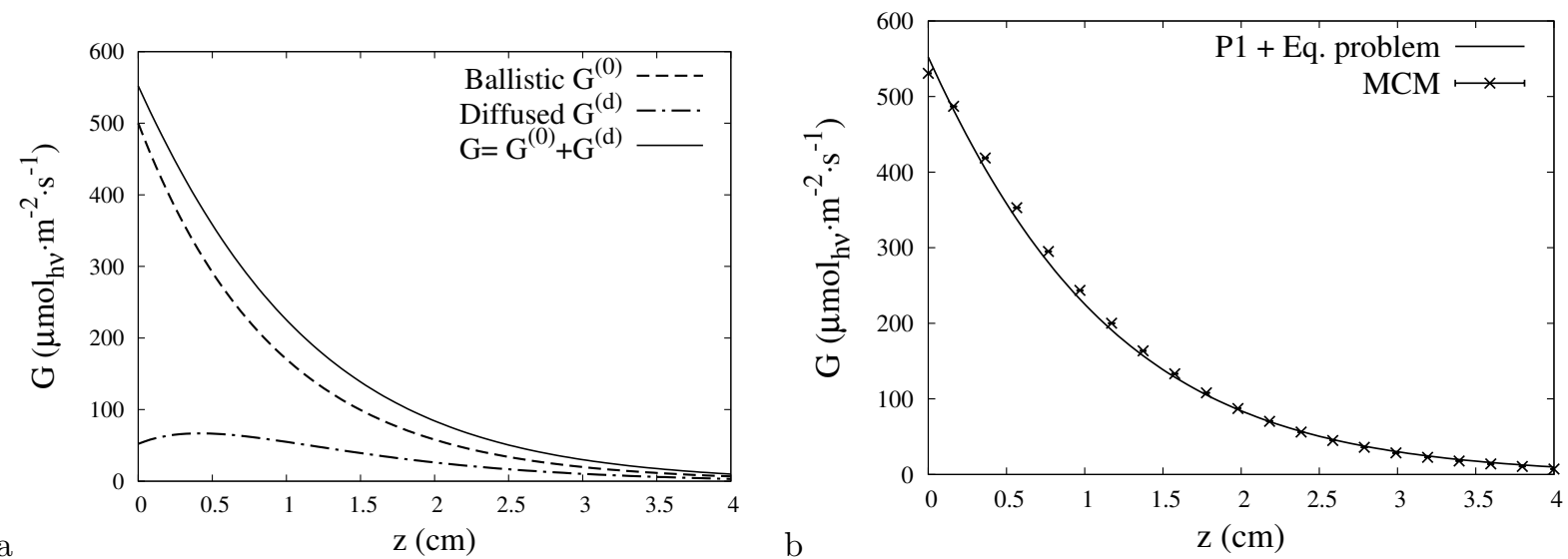

Figure 21: The irradiance field $G$ within the photobioreactor shown in Fig. $6 ; \rho^{\mathcal{F}}=\rho^{\mathcal{R}}=0$ and collimated normal incidence $\mu_{i}=1$. The results were obtained by means of the P1 approximation of the equivalent transport problem where $\alpha_{s}^{*}=0.25$, $k_{\text {ext }}^{*}=110 m^{-1}$, and $p_{\Omega}^{*}=1 / 4 \pi$ : the expression for $G^{(0)}$ is provided in Eq. 94, the expression for $G^{(d)}$ is shown in Eq. 96, and the total irradiance $G=G^{(0)}+G^{(d)}$ is shown in Eq. 102. a: The proportions of ballistic and diffused photons. b: Comparison with the reference solution obtained by the Monte Carlo method (MCM) for $\alpha_{s}=0.86, k_{e x t}=587 \mathrm{~m}^{-1}$, and the phase function of Chlamydomonas reinhardtii (see Section 4).
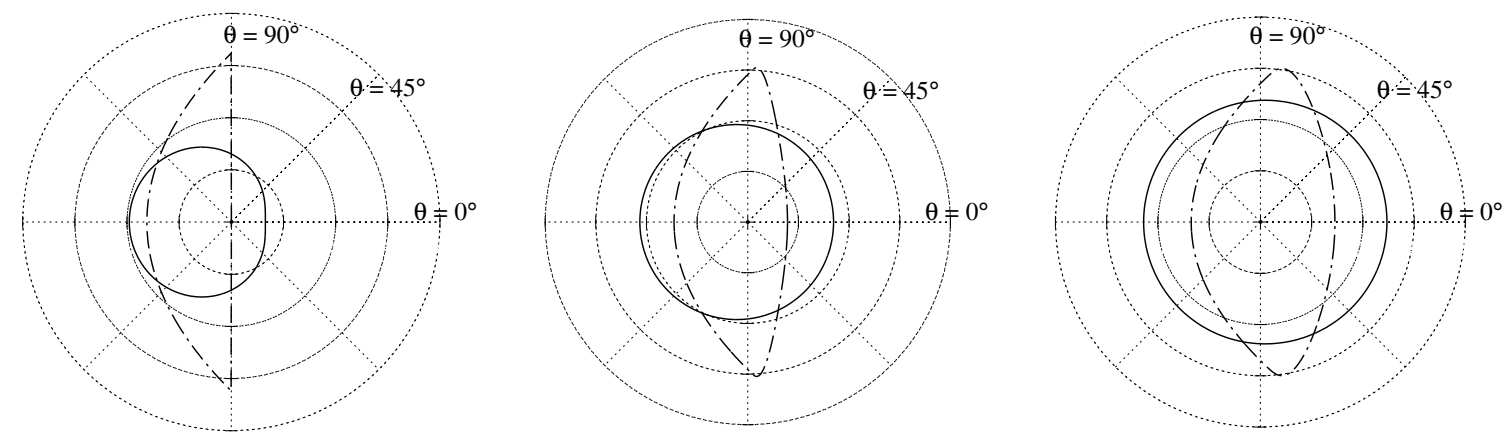

a

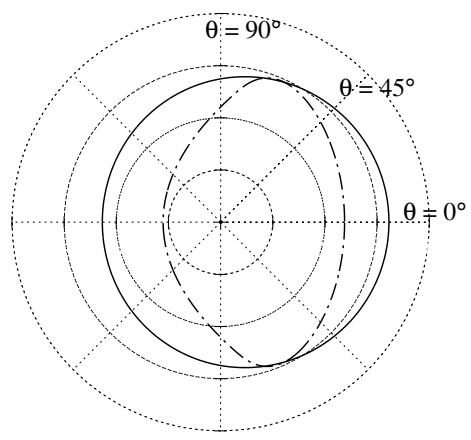

d

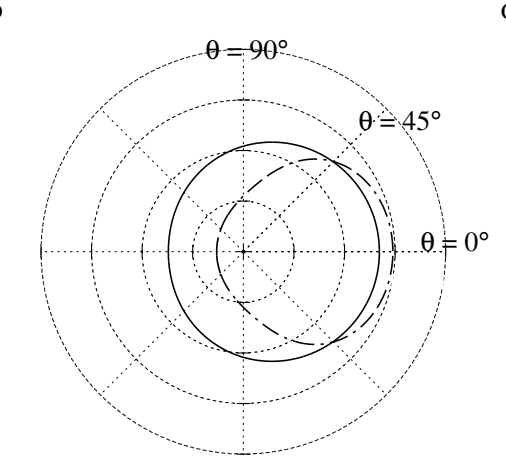

e

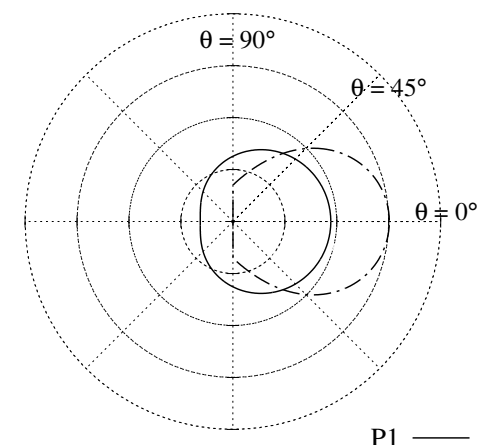

Single scattering

Figure 22: Angular distribution of the diffuse intensity $L^{(d)}\left(z_{0}, \theta\right)$ at the abscissa $z_{0}$ within the photobioreactor shown in Fig. 6; $\rho^{\mathcal{F}}=\rho^{\mathcal{R}}=0$; collimated normal incidence. The results were obtained for the equivalent transport problem where $\alpha_{s}^{*}=0.25$, $k_{\text {ext }}^{*}=110 \mathrm{~m}^{-1}$, and $p_{\Omega}^{*}=1 / 4 \pi$. Comparison between the $\mathrm{P} 1$ approximation and the single-scattering approximation (for which $L^{(d)} \simeq L^{(1)}$, see Section 3.3). a: $z_{0}=0$. b: $z_{0}=2.5 \mathrm{~mm}$. c: $z_{0}=5 \mathrm{~mm}$. d: $z_{0}=1 \mathrm{~cm}$. e: $z_{0}=2.5 \mathrm{~cm}$. f: $z_{0}=4 \mathrm{~cm}$. 
To sum up, the P1 approximation was shown to efficiently model the irradiance fields in typical flatplate photobioreactor configurations with intermediate optical-thickness values. Yet the validity condition of the P1 approximation is often associated with high scattering optical thickness (and with low absorption) because this criterion ensures situations near equilibrium. Nonetheless, P1 requires only that the angular dependence of the intensity can be formulated as the cosine of the propagation angle. Using a well-known invariance property of transport (in order to construct an equivalent transport problem), we proposed an approach enabling the use of the P1 approximation in a relevant manner in studies on photobioreactors, even for collimated incidence.

\subsection{Two-flux approximation}

This approximation is widely used for analytical purposes in spectroscopy (where it is called the KubelkaMunk theory), astrophysics, and photobioreactor engineering. Its implementation and capabilities in the context of photobioreactor research are detailed in $[2,4,6,7,8,11,27,70,71,72]$. The two-flux approximation is a macroscopic approximation of radiative transfer in the sense that it formulates a set of equations for description of the irradiance and flux density. Its advantage over other macroscopic approximations is that the assumption of near-equilibrium is not required. As in the case of the P1 approximation, the angular distribution of the intensity $L$ is fixed a priori, but here, $L$ does not have to be close to isotropy. Indeed, the functional form is fixed independently for forward and backward hemispheres, and this situation yields discontinuity at $\theta=\pi / 2$ (see Fig. 23). The approximation was originally developed under the assumption of isotropic intensity across both hemispheres [73] (see Fig. 23.a) and was then formulated for collimated intensity [74] (Dirac distributions in the forward and backward directions) and later extended to the intermediate anisotropic situation (e.g., see [75]). In the text below, we focus on a recent generalization [2, 6, 7] (developed in the context of photobioreactor engineering) that consists of the following functional form for the intensity, when applied to the configuration shown in Fig. 6:

$$
\begin{aligned}
& L(\mathbf{x}, \boldsymbol{\omega})=A^{+}(z)\left|\cos ^{n^{+}}(\theta)\right| \text { for } \theta \in[0, \pi / 2] \\
& L(\mathbf{x}, \boldsymbol{\omega})=A^{-}(z)\left|\cos ^{n^{-}}(\theta)\right| \text { for } \theta \in[\pi / 2, \pi]
\end{aligned}
$$

where $A^{+}(z)$ and $A^{-}(z)$ are functions of $z$, and the value of the parameters $n^{+}$and $n^{-}$determines the form of the angular distribution ( $n=0$ for isotropic intensity and $n \rightarrow \infty$ for collimated intensity). These parameters have to be fixed a priori and allow us to assume a wide range of the angular distribution with the same formula, as shown in Fig. 23.b. For example, different values can be chosen for $n^{+}$and $n^{-}$, leading to different assumptions for the angular distributions in the forward and backward hemispheres: Fig. 23.c represents the case of an isotropic distribution for one hemisphere $\left(n^{-}=0\right)$, and collimated for the other $\left(n^{+} \rightarrow \infty\right)$. Nevertheless, we observed very low sensitivity of the results to the value of $n^{-}$ for typical photobioreactor configurations. For this reason, we restrict the rest of our study to situations with $n^{+}=n^{-}=n$. Unlike with $\mathrm{P} 1$, the angular distribution here is identical within the whole reaction volume $\left(n^{+}\right.$and $n^{-}$are independent of the location) with discontinuity at the junction between the two hemispheres. On the basis of Fig. 17, we should note that this discontinuity may be justified at the boundary of the system, but within the volume, this assumption is plausible only for optically thin media $\left(i . e ., e_{s}^{*} \ll 1\right)$, or equivalently, for locations close to the boundaries.

Substituting Eq. 104 into the radiative transfer equation Eq. 24 (in which we omit the frequency variable) and integrating over propagation directions $\boldsymbol{\omega}$ (i.e., over the propagation angles $\theta$ ), we obtain the following equation for the irradiance within the photobioreactor shown in Fig. 6 with $\rho^{\mathcal{F}}=0$ [2]:

$$
\begin{aligned}
& G(z)=\frac{q_{\cap}}{\mu_{i}} 2 \frac{n+2}{n+1} \times \\
& \frac{\rho^{\mathcal{R}}(1+\alpha) \exp (-\delta E)-(1-\alpha) \exp (-\delta(E-z))+\left[(1+\alpha) \exp (\delta E)-\rho^{\mathcal{R}}(1-\alpha) \exp (\delta E)\right] \exp (-\delta z)}{(1+\alpha)^{2} \exp (\delta E)-(1-\alpha)^{2} \exp (-\delta L)+\rho^{\mathcal{R}}(1-\alpha)^{2}[\exp (-\delta E)-\exp (\delta E)]}
\end{aligned}
$$


where

$$
\begin{gathered}
\alpha=\sqrt{\frac{\sigma_{a}}{\sigma_{a}+2 b \sigma_{s}}} \\
\delta=\frac{\alpha}{\mu_{i}} C x\left(\sigma_{a}+2 b \sigma_{s}\right)
\end{gathered}
$$

where $\mu_{i}$ is the cosine of the incidence angle $\theta_{i}, b$ is the backscattering coefficient

$$
b=2 \pi \int_{\pi / 2}^{\pi} p\left(\theta_{s}\right) \sin \left(\theta_{s}\right) d \theta_{s}
$$

i.e., the integral of the phase function $p$ over backscattering directions (see Section 2 and Fig. 2), $b=0.008$ for the phase function in question, and all other notations are defined in Fig. 6.

In Fig. 24, the irradiance field obtained with the two-flux approximation for $n \rightarrow \infty$ is compared with the Monte Carlo reference solution in the case of collimated solar-light incidence. The two-flux approximation will be used in Section 5.6 to analyze the coupling between radiative transfer and photosynthesis thermokinetics in photobioreactors with simple geometric structure. 

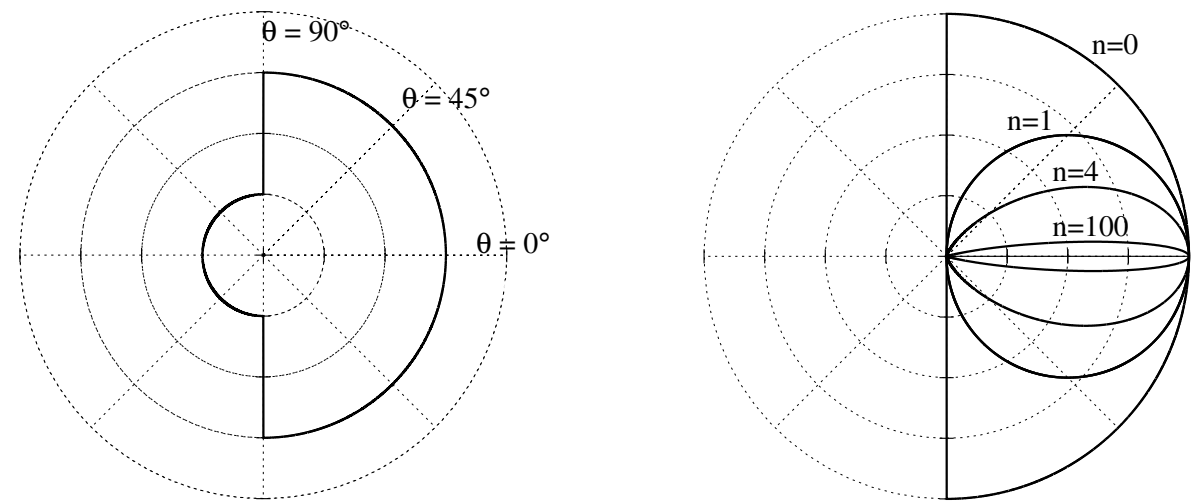

a

$\mathrm{b}$

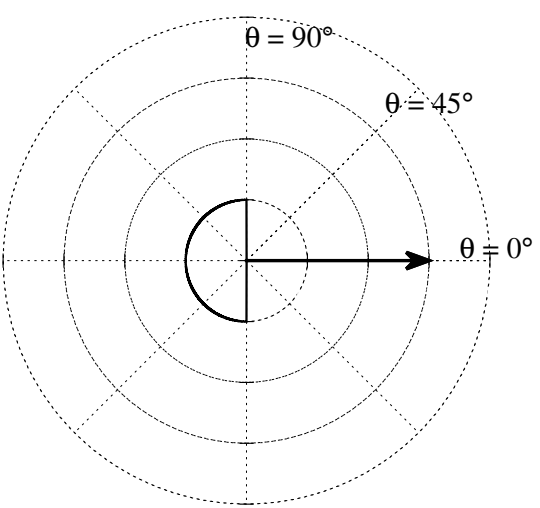

c

Figure 23: The two-flux approximation: different angular distributions of the intensity that can be postulated. a: Isotropic distribution for each hemisphere. b: Distribution (Eq. 104) for $\theta \in[0, \pi / 2]$ at different values of $n$. c: Collimated distribution for one hemisphere and isotropic distribution for the other.

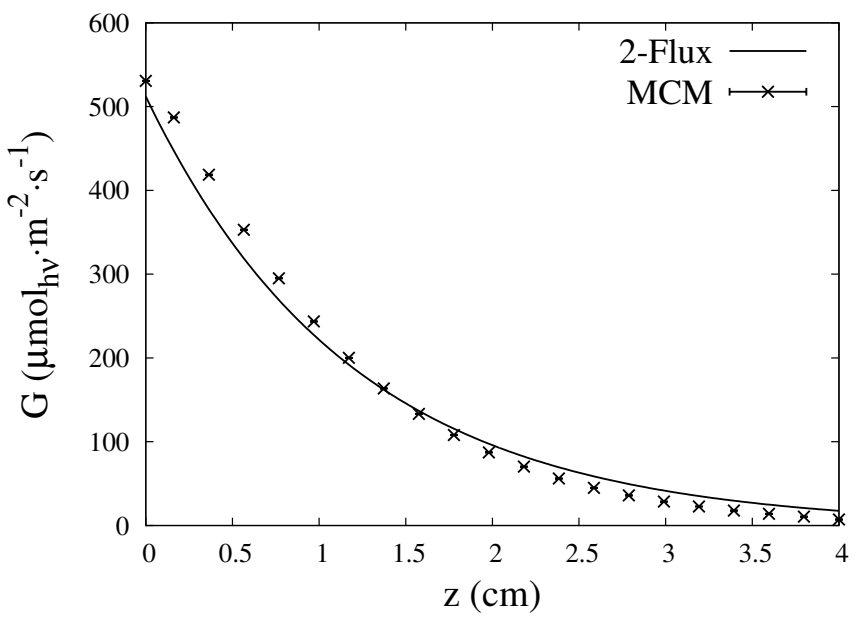

Figure 24: The irradiance field $G$ in the photobioreactor shown in Fig. $6 ; \rho^{\mathcal{F}}=\rho^{\mathcal{R}}=0$ and collimated normal incidence $\mu_{i}=1$. Comparison between the two-flux approximation (Eq. 105) at $b=0.008$ and the reference solution obtained by the Monte Carlo method (MCM; see Section 4). 
3.6. Implementation of the analytical approximate solutions developed in this section for the field of specific absorption rate $\mathcal{A}$

During the previous radiative-transfer analysis, the following approximate solutions were obtained for the irradiance field within the typical photobioreactor configuration in Fig. 6:

1. The single-scattering approximation applied to the equivalent transport problem for analysis of collimated illumination in the case of non-reflecting surfaces: Eqs. 65 to 68.

2. The P1 approximation for analysis of diffuse illumination: Eq. 88.

3. The P1 approximation applied to the equivalent transport problem for analysis of collimated illumination in the case of non-reflecting surfaces: Eq. 102.

4. The two-flux approximation: Eq. 105.

These solutions can be used in various ways to calculate the field of specific absorption rate $\mathcal{A}$ :

- In the numerical calculations presented in the figures of this section, we used the approximation of an equivalent gray medium (see Eqs. 35 and 36). In this case, the gray radiative properties are used directly in the expressions, and the variable $q_{\cap}$ is the value of the incident surface flux density (integrated over PAR). Finally, the solution for the irradiance is simply multiplied by the gray absorption cross-section to obtain the specific rate of photon absorption $\mathcal{A}=\sigma_{a} G$ (see the discussion at the end of Section 3.1). This approach allows us to obtain simple analytical solutions appropriate for such analysis.

- On the other hand, the spectral integration can also be analyzed. In this case, the spectral radiative properties are used in the expressions, and the variable $q_{\cap}$ assumes the value of the spectral distribution $q_{\cap, \nu}$ of the incident flux density (see Eq. 25). This situation leads to an expression for the spectral distribution $G_{\nu}$ of the irradiance, which is multiplied by the spectral absorption cross-section and integrated over PAR in order to obtain the specific rate of photon absorption: $\mathcal{A}=\int_{\nu_{\min }}^{\nu_{\max }} \sigma_{a, \nu} G_{\nu} d \nu$ (see Eq. 31).

In both of the above cases, $\mathcal{A}$ and $G$ (according to Section 3.1) can be expressed

- either in moles of photons per second if the variable $q_{\cap}$ is expressed in moles of photons per second,

- or in Watts if the variable $q_{\cap}$ is expressed in Watts.

It should be noted that the approximations explored in this section can also be used to obtain analytical solutions for one-dimensional cylindrical configurations (e.g., see [2] regarding the case of the two-flux approximation). Moreover, in the case of solutions 1 and 3 in the above list, we chose to focus on nonreflecting surfaces in order to simplify the mathematical expressions. These approximations, however, are not restricted to non-reflecting surfaces. For example, when extended to reflecting surfaces on both sides, solution 3 is still analytical.

It is also important to note that due to the linearity of the radiative transfer equation, the solutions for configurations illuminated on both sides (or for mixtures of collimated and diffuse illumination) are obtained simply by adding up the solutions obtained in this section. For example, for incident solar radiation with direct and diffuse components, the radiation field can be obtained by adding up solutions 2 and 3 . For a photobioreactor illuminated on both sides, the radiation field is $G(z)=\tilde{G}(z)+\tilde{G}(E-z)$, where $\tilde{G}$ is any solution obtained for an emitting surface at $z=0$. Again, these solutions can be linearly combined in order to analyze a photobioreactor illuminated on both sides by a mixture of collimated and diffuse radiation. 


\section{Numerical implementation of photobioreactor models by the Monte Carlo method, includ- ing rigorous solution of the radiative transfer equation for complex geometric structure}

Since Metropolis' original work in 1949 [76], numerous monographs and review articles have been devoted to the Monte Carlo method. In the present study, we are concerned both with simulation of a linear transport phenomenon (namely radiative transfer) and with a solution to our integral model for a photobioreactor (see Section 1). Here, we arbitrarily chose to point out J.M. Hammersley and D.C. Handscomb's book [77] because of the everlasting influence of this short synthesis on this area of research as well as J.R. Howell's review [78] because of its proximity to our more specific engineering-application concerns in the field of radiative transfer. These texts provide a sufficient theoretical framework for most of the algorithms encountered in photobioreactor research and may serve as a meaningful starting point for any further bibliographic research.

Among more recent methodological advances, simulation of nonlinear processes, sensitivity estimation, and the zero-variance concept are discussed in Section 4.3, Section 4.4, and [79, 80], respectively, because they can be at least partially translated into simple systematic procedures for simulation and analysis of photobioreactor models. They rely on explicit definition of the strict relation between a linear Monte Carlo algorithm and an integral formulation. Indeed, the Monte Carlo method is, above all, a numerical approach to solving integrals. In our context, this approach implies that the method is not only pertinent to simulation of radiative transfer (it is generally thought to be the reference method for solution of the radiative transfer equation) but also very well suited for solving our photobioreactor model, which is based on integral formulations. Let us briefly illustrate this relation between the Monte Carlo algorithms and integral formulations with a simple example that does not imply radiative transfer: estimation of the average rate of biomass production $\left\langle r_{x}\right\rangle$ in a photobioreactor, as formulated in Eq. 1 (Section 1):

$$
<r_{x}>=\int_{\mathcal{V}} \frac{1}{V} r_{x}\left(\mathbf{x}_{0}\right) d \mathbf{x}_{0}
$$

where the local rate $r_{x}$ is assumed to be known at any location $\mathbf{x}_{0}$ (for illustration purposes). This integral formula can be interpreted statistically as the expectation of the random variable $W=\hat{w}\left(\mathbf{X}_{0}\right)=r_{x}\left(\mathbf{X}_{0}\right)$, where $\mathbf{X}_{0}$ is a random location within $\mathcal{V}$, with uniform probability density function $p_{\mathbf{X}_{0}}=\frac{1}{V}$ :

$$
<r_{x}>=\int_{\mathcal{V}} p_{\mathbf{x}_{0}} \hat{w}\left(\mathbf{x}_{0}\right) d \mathbf{x}_{0}
$$

The corresponding Monte Carlo algorithm consists of sampling $N$ independent realizations $w_{1}$ and $w_{2} \cdots w_{N}$ of the random variable $W$ by repeating $N$ times the following sampling procedure (where $i=1,2 \cdots N$ ): Step (1) A location, $\mathbf{x}_{0}$, is sampled within $\mathcal{V}$ according to the probability density function $p_{\mathbf{X}_{0}}=\frac{1}{V}$ (i.e., uniform sampling).

Step (2) The weight $w_{i}$ is calculated according to $w_{i}=r_{x}\left(\mathbf{x}_{0}\right)$.

Then, $\left\langle r_{x}\right\rangle$ is estimated as

$$
<r_{x}>\simeq \tilde{b}_{N}=\frac{1}{N} \sum_{i=1}^{N} w_{i}
$$

Because realizations $w_{i}$ are independent, meaningful statistical uncertainty (evaluation of the standard deviation of the estimator) is systematically available as

$$
\tilde{\sigma}_{N}=\frac{1}{\sqrt{N-1}} \sqrt{\left(\frac{1}{N} \sum_{i=1}^{N} w_{i}^{2}\right)-\tilde{b}_{N}^{2}}
$$

that is, directly related to the numerical error. In general terms, during analysis of the physical quantity $B$ (in our example $B=<r_{x}>$ ), any approximation $\tilde{b}_{N}$ of $B$ corresponding to a linear Monte Carlo algorithm involving $N$ sampled events is constructed as Eq. 110, with the statistical uncertainty Eq. 111 . The events 
can be simple, as in our example. In contrast, as illustrated in Section 4.1, the events rapidly become quite complex as soon as radiative transfer in multiple-reflection and multiple-scattering configurations is simulated. In all cases, however, the reason why the Monte Carlo method is so popular is its intuitive nature: in the above example, the average production rate $\left\langle r_{x}\right\rangle$ is estimated simply as the average of $N$ local production rates evaluated at uniformly sampled locations. The method is nonetheless mathematically rigorous: the meaning of the integral formulation Eq. 109 is that when $N \rightarrow+\infty$, the estimator $\tilde{b}_{N}$ evaluates $\left\langle r_{x}\right\rangle$ as the expectation of the random variable $W$. When we simulate radiative transfer (i.e., when $B$ is a radiative quantity), the events are more complex, but these advantages (mathematical rigor and the ease of understanding) are preserved: the integral solution of the radiative transfer equation is estimated by "tracing photon trajectories" in the photobioreactor.

In the above example, the local production rate $r_{x}$ was assumed to be known for the purposes of illustration. Nevertheless, as stated in Section 1 (and detailed in Section 5), $r_{x}$ is a function of the specific rate of photon absorption $\mathcal{A}$. This is why photobioreactor studies require solution of the radiative transfer equation prior to estimation of the production rate $\left\langle r_{x}\right\rangle$. A radiative-transfer Monte Carlo algorithm for rigorous estimation of $\mathcal{A}$ is presented in Section 4.1, and its implementation for complex geometric structure is discussed in Section 4.2. Based on this algorithm, the estimation of the production rate $\left\langle r_{x}\right\rangle$ of photobioreactors is addressed in Section 4.3. Finally, in Section 4.4, we briefly explore the expected benefits of sensitivity estimation, in relation to the analysis and optimization of the process.

Monte Carlo integral-formulations such as Eq. 109 lie at the root of the work that is presented below. Nevertheless, we chose to avoid the details of these kinds of formulations in the text that follows because we believe that they are beyond the scope of the present book: integral formulae will be used for illustrative purposes only. Readers wishing to explore the formal basis of our work more deeply are invited to read [79, $80]$.

\subsection{An algorithm for evaluating the specific rate of photon absorption}

In this section, we present a Monte Carlo algorithm for estimation of the specific photon absorption rate $A\left(\mathbf{x}_{0}\right)$ at any location $\mathbf{x}_{0}$ within any photobioreactor's reaction volume confined by two diffuse-reflective surfaces $\left(\mathcal{R}\right.$ and $\mathcal{F}$ ) with uniform reflectivity $\rho^{\mathcal{R}}$ and $\rho^{\mathcal{F}}$, respectively, where $\mathcal{F}$ is Lambertian emitting with uniform surface flux density $q_{\cap, \nu}$ and $\mathcal{R}$ is non-emitting. Let us recall the definition of $A\left(\mathbf{x}_{0}\right)$ from Section 3.1:

$$
\mathcal{A}\left(\mathbf{x}_{0}\right)=\int_{\nu_{\min }}^{\nu_{\max }} d \nu \int_{4 \pi} d \boldsymbol{\omega}_{0} \sigma_{a, \nu} L_{\nu}\left(\mathbf{x}_{0},-\boldsymbol{\omega}_{0}\right)
$$

where $L_{\nu}\left(\mathbf{x}_{0},-\boldsymbol{\omega}_{0}\right)$ is the intensity at $\mathbf{x}_{0}$ in the direction $-\boldsymbol{\omega}_{0}$ at frequency $\nu$. You may recall that the integral over the reaction volume was translated into a location-sampling procedure in our introductory example. In exactly the same way, the Monte Carlo algorithm for estimation of Eq. 112 starts with the sampling of a frequency, that is, with translation of the integral over PAR $\left[\nu_{\min }, \nu_{\max }\right]$, followed by sampling of a direction $\left(\boldsymbol{\omega}_{0}\right)$, that is, translation of the integral over the total solid angle $4 \pi$. Then, in order to estimate $L\left(\mathbf{x}_{0},-\omega_{0}\right)$, we design a reverse Monte Carlo procedure consisting of sampling of a multiple scattering and reflection optical path starting from $\mathbf{x}_{0}$ with direction $\omega_{0}$ until it is "absorbed" at the emitting surface $\mathcal{F}$ (i.e., the optical paths are sampled backward). The sampling procedure is detailed next and illustrated in Fig. 25 with two examples: the one-dimensional photobioreactor from Fig. 6 and a prototype of the volumetrically illuminated photobioreactor from Fig. 26. The corresponding integral formulation is reported in [79, 80].

The sampling procedure:

Step (1) A frequency is sampled across $\left[\nu_{\min }, \nu_{\max }\right]$ (PAR) according to the uniform probability density function $p_{\nu}(\nu)=\frac{1}{\nu_{\max }-\nu_{\min }}$. This frequency determines all the spectral properties for the current optical path: scattering and absorption properties of the reaction volume (i.e., the radiative properties calculated in Section 2), reflectivity of surfaces, and surface flux density $q_{\cap, \nu}$ emitted at the surface $\mathcal{F}$.

Step (2) Starting from the location $\mathbf{x}_{0}$, the first propagation direction $\omega_{0}$ is sampled across the total solid angle according to the isotropic probability density function $p_{\Omega_{0}}^{\mathcal{V}}\left(\omega_{0}\right)=\frac{1}{4 \pi}$, and the first scattering length $l_{0}$ is sampled across $[0,+\infty]$ according to the Beer extinction law $p_{L_{0}, \nu}\left(l_{0} ; k_{s, \nu}\right)=k_{s, \nu} \exp \left(-k_{s, \nu} l_{0}\right)$, where $k_{s, \nu}$ is the scattering coefficient calculated in Section 2. 
Step (3) Now that $\left\{\mathbf{x}_{0}, \omega_{0}, l_{0}\right\}$ has been sampled, the first interaction location $\mathbf{x}_{1} \equiv \mathbf{x}_{1}\left(\mathbf{x}_{0}, \omega_{0}, l_{0}\right)$ is determined. As discussed in Section 4.2, purely geometric considerations are easily translated into scientific computation libraries. For a given couple, $\left\{\mathbf{x}_{0}, \omega_{0}\right\}$, such libraries provide us with the location $\mathbf{y}_{1} \equiv \mathbf{y}_{1}\left(\mathbf{x}_{0}, \omega_{0}\right)$ of the first time the half-line (starting at $\mathbf{x}_{0}$ in the direction $\omega_{0}$ ) intersects the total bounding surface $\mathcal{R} \cup \mathcal{F}$ (see Fig. 25.a). If the distance $\left\|\mathbf{y}_{1}-\mathbf{x}_{0}\right\|$ to the bounding surface is smaller than the scattering length $l_{0}$, then the optical path interacts with the surface (see Fig. 25.b); otherwise, scattering occurs inside the volume of culture (Fig. 25.a):

$$
\mathbf{x}_{1}= \begin{cases}\mathbf{y}_{1} & \text { if }\left\|\mathbf{y}_{1}-\mathbf{x}_{0}\right\|<l_{0} \\ \mathbf{x}_{0}+l_{0} \omega_{0} & \text { otherwise }\end{cases}
$$

Step (4) A branching test is performed depending on the nature of the interaction:

- In case of an interaction with the non-emitting surface $\mathcal{R}$ of the photobioreactor, the Bernoulli test is performed: a random number, $r_{1}$, is uniformly sampled across the unit of interval and

- if $r_{1}$ is less than the surface reflectivity $\rho^{\mathcal{R}}$, then the optical path is reflected (see Fig. 25.b): the reflection direction $\omega_{1}$ is sampled according to the diffuse angular distribution $p_{\boldsymbol{\Omega}_{1}}^{\mathcal{R}}\left(\omega_{1}\right)=\frac{\omega_{1} \cdot \mathbf{n}_{1}}{\pi}$ $\left(\mathbf{n}_{1}\right.$ being the normal at the location $\left.\mathbf{x}_{1}\right)$, and a new scattering length $l_{1}$ is sampled according to the same extinction law as for $l_{0}\left(p_{L_{1}, \nu} \equiv p_{L_{0}, \nu}\right)$;

- otherwise, the optical path sampling procedure is terminated and the weight $\hat{w}_{1}$ is calculated according to Eq. 113 (in this case, the photon is dissipated at the reflecting surface and therefore $\left.\hat{w}_{1}=0\right)$.

- In case of an interaction with the emitting surface $\mathcal{F}$, a Bernoulli test is performed: a random number, $r_{1}$, is uniformly sampled across the unit of the interval and

- if $r_{1}$ is less than the surface reflectivity $\rho^{\mathcal{F}}$, then the optical path is reflected (see Fig. 25.c): the reflection direction $\omega_{1}$ and the scattering length $l_{1}$ are sampled as described above $\left(p_{\boldsymbol{\Omega}_{1}}^{\mathcal{R}} \equiv p_{\boldsymbol{\Omega}_{1}}^{\mathcal{F}}\right)$;

- otherwise, the optical-path-sampling procedure is terminated, and the weight $\hat{w}_{1}$ is calculated according to Eq. 113 (in this case, the optical path contributes to local absorption, and therefore $\hat{w}_{1}$ is not zero).

- Finally, if $\mathbf{x}_{1}$ is within the volume of culture $\mathcal{V}$, a scattering direction $\left(\omega_{1}\right)$ is sampled according to the single-scattering phase function $p_{\Omega_{1}, \nu}^{\mathcal{V}}\left(\omega_{1} \mid \omega_{0}\right)$ calculated in Section 2, and $l_{1}$ is sampled as described above (see Fig. 25.d).

Step (5) At this stage, if the optical-path-sampling procedure is not terminated, the algorithm loops to (3) and evaluates the next interaction position (the index 1 being incremented to 2 , and the index 0 being incremented to 1) and so on until absorption occurs at the surface $\mathcal{R}$ or $\mathcal{F}$.

An example of an optical path sampled by this procedure is shown in Fig. 26.c. Altogether, each sampled optical path leads to evaluation of the weight according to the weight function $\hat{w}_{j}$ (see below), and $A\left(\mathbf{x}_{0}\right)$ is estimated as the average of all weights.

$$
\begin{array}{ll}
\hat{w}_{j}=0 & \text { if } \mathbf{x}_{j} \in \mathcal{R} \\
\hat{w}_{j}=\left(\nu_{\max }-\nu_{\min }\right) 4 \pi \sigma_{a, \nu} \frac{q_{\cap, \nu}}{\pi\left(1-\rho^{\mathcal{F}}\right)} e^{-k_{a, \nu} d_{j}} & \text { if } \mathbf{x}_{j} \in \mathcal{F}
\end{array}
$$

where $\left[\nu_{\min }, \nu_{\max }\right]$ is PAR, $\sigma_{a, \nu}$ is the absorption cross-section ${ }^{10}$ determined in Section $2, q_{\cap, \nu}$ is the spectral distribution of the surface flux density emitted at $\mathcal{F}$ (see Section 3.1), $\rho^{\mathcal{F}}$ is reflectivity of $\mathcal{F}, q_{\cap, \nu} / \pi\left(1-\rho^{\mathcal{F}}\right.$ ) is the equivalent blackbody intensity for emission at $\mathcal{F}$, and $e^{-k_{a, \nu} d_{j}}$ is the transmission along the optical

\footnotetext{
${ }^{10}$ In Section 3, we estimate the local irradiance $G\left(\mathbf{x}_{0}\right)$ by the same algorithm, only replacing $\hat{w}_{j}$ with the new weight function $\hat{w}_{j}^{G}=\hat{w}_{j} / \sigma_{a, \nu}$.
} 
path (accounting for attenuation due to absorption), with $d_{j}=\sum_{q=0}^{j-1}\left\|\mathbf{x}_{q+1}-\mathbf{x}_{q}\right\|$ being the total length of the sampled optical path.

The Monte Carlo method is usually preferred to other numerical simulation approaches because of its flexibility (in terms of inclusion of new physical phenomena) and its ability to deal with geometrically complex realistic systems. Next, we briefly illustrate the flexibility of the above algorithm; analysis of complex geometric structures is specifically addressed in Section 4.2. Monte Carlo codes for standard radiative transfer can be depicted as close translations of well-established physical situations of photon emission, scattering, reflection, refraction and absorption. Therefore, such codes are indeed easy to design and easy to upgrade toward representation of additional (or more accurate) physical phenomena. For example, the above algorithm can be easily modified for analysis of specular-reflective surfaces: in this case, at Step (4), the reflection direction $\boldsymbol{\omega}_{1}$ is set to the specular direction corresponding to the incident direction $\boldsymbol{\omega}_{0}$ (see Fig. 10) instead of sampling $\boldsymbol{\omega}_{1}$ according to the diffuse angular distribution. Extension of our algorithm to heterogeneous illumination is also straightforward (e.g., simulation of a tubular photobioreactor): the very same algorithm is used except in the weight function (Eq. 113), the homogeneous surface flux density $q_{\cap, \nu}$ is replaced by the surface flux density $q_{\cap, \nu}\left(\mathbf{x}_{j}\right)$ at location $\mathbf{x}_{j}$. Among all the possible refinements of our algorithm, the addition of solar-light collection (accounting, for example, for shading and blocking effects or collector orientation) is certainly an interesting perspective. In this case, the optical-path-sampling procedure does not stop at the surface $\mathcal{F}$ but continues (e.g., within the atmosphere) according to standard Monte Carlo algorithms for concentrated solar applications, such as those presented in [79].

\subsection{Practical implementation for complex geometric structure}

In this section, we address the practical question of numerically implementing the above algorithm within photobioreactors with complex geometric structure. Fig. 25 shows an example of a DiCoFluV photobioreactor [7], in which the incident solar-light flux density is diluted within the volume of culture because of a thousand light-diffusing optical fibers emitting a quasi-homogeneous density flux on the totality of their surface (see Fig. 26).

With the Monte Carlo method, the difficulty with the geometric complexity is reduced to calculation of the intersections between the straight rays and surfaces. In Section 4.1, this situation corresponds to calculation of the intersection between half-lines starting at $\mathbf{x}_{j}$ in the direction $\omega_{j}$ and the surface $\mathcal{R} \cup \mathcal{F}$ [Step (3) of our algorithm]. Indeed, the calculation of these intersections for the complex surface $\mathcal{R} \cup \mathcal{F}$ of a DiCoFluV is the only additional difficulty, in contrast to the implementation for a one-dimensional Cartesian photobioreactor (see Fig. 25).

In $[79,80]$, this practical difficulty is alleviated by implementation in the EDStar development environment [56], which makes available to radiation physicists a set of computational tools produced by the computer graphics research community during the last 20 years, in particular within the framework of the Physically Based Rendering Techniques (PBRT) project [81]. These tools are helpful in the process of geometric definition of complex scenes and for accelerating photon tracking in such scenes. The implementation in EDStar completely separates the geometry and physics: the geometric structure is defined (or imported) in a specific file, whereas the Monte Carlo algorithm describing the physical phenomena (such as absorption, scattering, and reflection) is written in a separate file where researchers have access to abstractions such as ray, intersection, or shape that are used to code the optical-path-sampling procedure regardless of the geometric structure specified in the first file. This separation perfectly meets the needs of the modern engineering studies: first, the algorithm is developed without worries about the technical characteristics that have no direct relation to physical reasoning (EDStars scientific computation libraries handle statistical methods, parallelization, and pure geometric questions), then the algorithm is validated in simple geometric structure, and finally it can be directly implemented for any geometric structure of a photobioreactor (without modification of the sampling procedure).

Both the file for the optical-path-sampling algorithm corresponding to the procedure in Section 4.1 and the scene description file corresponding to the DiCoFluV photobioreactor in Fig. 26 are provided on the EDStar website [56]. This code was used in [79, 80] to estimate the specific absorption rate at any location within the DiCoFluV. For estimation with $1 \%$ accuracy, we observed calculation time of $\sim 5 s$ per location with the processor Quad-Core Intel Xeon $2.66 \mathrm{GHz}$ (the time decreases linearly with the number 

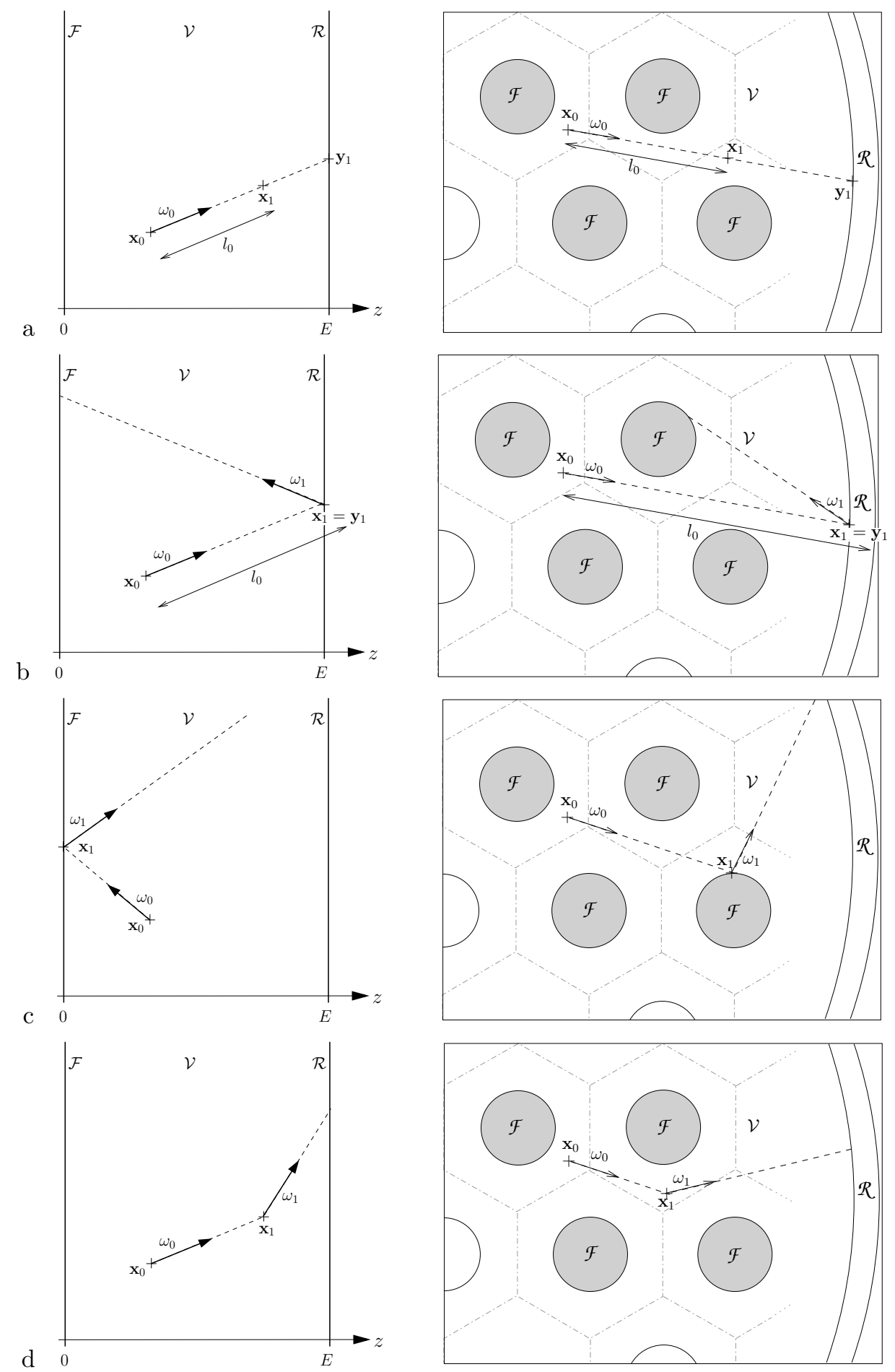

Figure 25: Illustration of the Monte Carlo algorithm presented in Section 4.1 for evaluation of the local rate of photon absorption $\mathcal{A}\left(\mathbf{x}_{0}\right)$ at any location $\mathbf{x}_{0}$ within the culture volume: (left panel) the case of the one-dimensional Cartesian configuration shown in Fig. 6; (right panel) the case of the DiCoFluV photobioreactor presented in Fig. 26. 
of processors in a parallel implementation). Additionally, Fig. 27 shows the influence of the number of optical fibers in the photobioreactor. These results indicate that because of the computer graphics tools for acceleration of ray tracing, the calculation time is independent of the geometric complexity. These results were obtained for the configuration presented in Fig. 26, in which both the photobioreactor $\mathcal{R}$ and fibers $\mathcal{F}$ are modeled as cylinders. In [82], we tested our ability to extend this practice to simulation of performance of a photobioreactor on the basis of its computer-aided design (CAD), which is a tool used by engineers in the process of designing an innovative reactor, from initial sketching to final realization. We imported the CAD of the DiCoFluV prototype into EDStar and estimated the radiation field within this geometric structure, which is composed of 73000 triangles describing the surfaces of fibers and a stainless-steel vessel. Common bugs encountered with such complex geometric structure were tracked, in particular, photon losses at the triangles' edges. No major difficulties were encountered in the present case, and the stability of calculation time was preserved.

Therefore, available computer graphics tools allow us to simulate any geometric structure of a photobioreactor with the same Monte Carlo algorithm and with acceptable calculation time. This recent advance in the field of the Monte Carlo method opens up interesting perspectives on photobioreactor design, for example, in relation to the optimization algorithm.
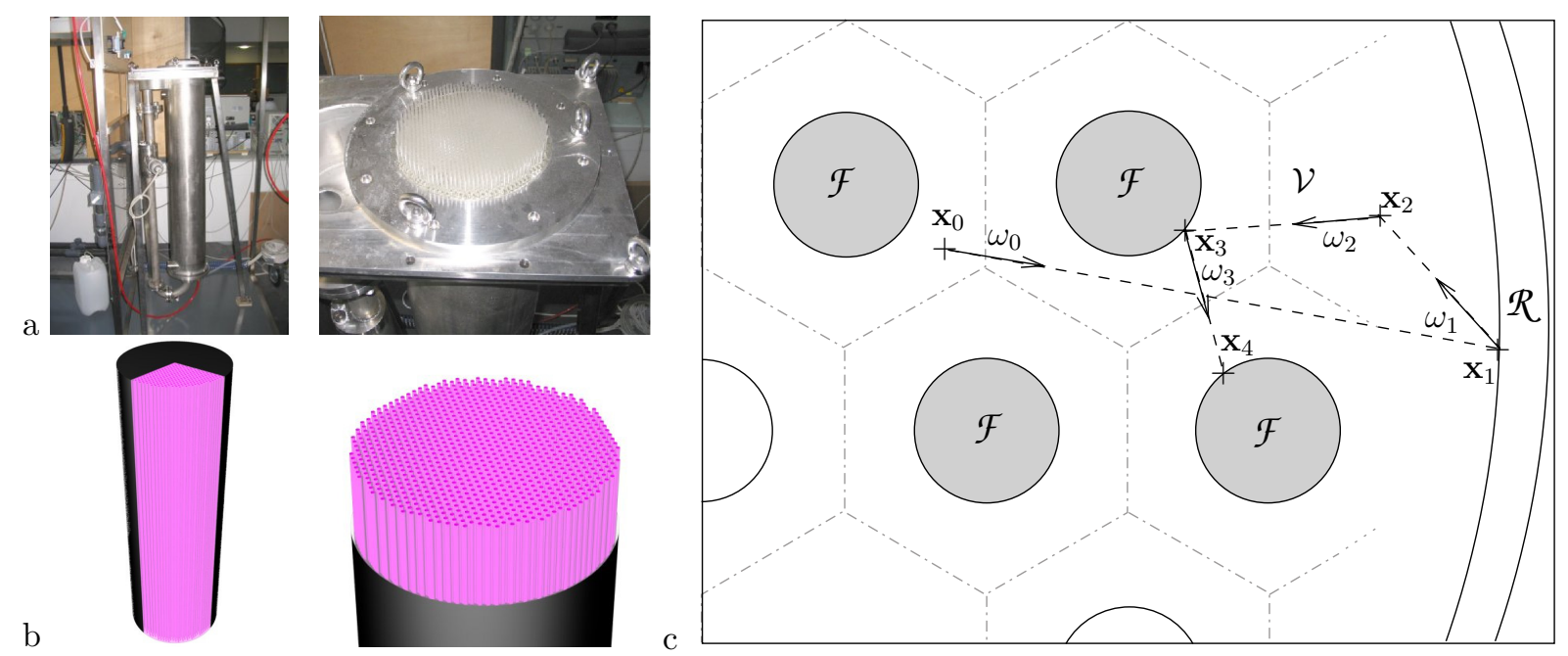

Figure 26: a: A 25L prototype of the solar volumetrically illuminated photobioreactor DiCoFluV [7]. b: EDStar geometric structure: both the reactor $(\mathcal{R})$ and the 979 light-diffusing optical fibers $(\mathcal{F})$ are cylinders $1 m$ high; the reactor's diameter is $16.5 \mathrm{~cm}$; the distance between two fiber axes is $d_{\mathcal{F}}=4.8 \mathrm{~mm}$; the fiber radius is $r_{\mathcal{F}}=1.2 \mathrm{~mm} . \mathcal{R}$ and $\mathcal{F}$ are diffuse-reflective with uniform reflectivity $\rho^{\mathcal{R}}$ and $\rho^{\mathcal{F}}$, respectively. $\mathcal{F}$ is Lambertian emitting with the uniform surface flux density $q \cap, \nu$. c: Two-dimensional hexagonal lattice fiber arrangement; an optical-path example in the culture medium $\mathcal{V}$.

\subsection{Coupling of radiative transfer with photosynthesis}

The following paragraphs address estimation of the production rate $\left\langle r_{x}\right\rangle$ of a photobioreactor. To be precise, we discuss how a recent methodological advance in the simulation of nonlinear processes by the Monte Carlo method allows us to estimate $\left\langle r_{x}\right\rangle$ without constructing the field of the absorption rate $\mathcal{A}$ within the reaction volume. On the one hand, this method obviates the cumbersome task of constructing an appropriate volume mesh for estimation of the $\mathcal{A}$ field; this approach is especially convenient in the case of internally lightened technologies such as DiCoFluV. On the other hand, this approach allows researchers to rigorously evaluate $\left\langle r_{x}\right\rangle$ with acceptable calculation time (a few minutes in the case of DiCoFluV).

Among the numerical methods for evaluation of integrals, the Monte Carlo method is famous because its convergence is independent of the dimension of the integration domain [77]. In the present context, this means, for example, that the calculation time for evaluating the average absorption rate $<\mathcal{A}>=$ 


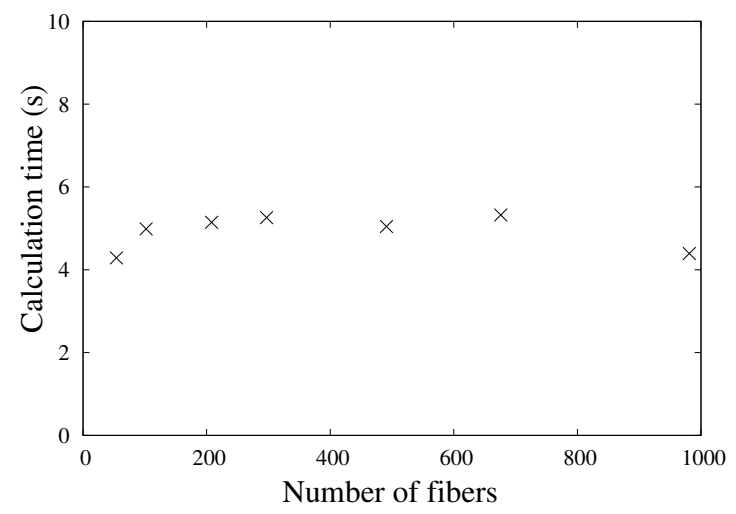

Figure 27: Calculation time for estimating the specific rate of photon absorption $\mathcal{A}$ within the reaction volume of the DiCoFluV photobioreactor, as a function of the number of light-diffusing optical fibers. The results were obtained in EDStar [79] by implementation of the algorithm presented in Section 4.1 for different versions of the geometric structure in Fig. 26.b (containing different numbers of fibers).

$\int_{\mathcal{V}} d \mathbf{x}_{0} \frac{1}{V} \mathcal{A}\left(\mathbf{x}_{0}\right)$ is almost the same as that for evaluating $\mathcal{A}\left(\mathbf{x}_{0}\right)$ at one location $\mathbf{x}_{0}$. This is because the integration domains are linearly combined in the integral formula for $\langle\mathcal{A}\rangle$ :

$$
<\mathcal{A}>=\int_{\mathcal{V}} d \mathbf{x}_{0} \frac{1}{V} \int_{\nu_{\min }}^{\nu_{\max }} d \nu \int_{4 \pi} d \boldsymbol{\omega}_{0} \sigma_{a, \nu} L_{\nu}\left(\mathbf{x}_{0},-\boldsymbol{\omega}_{0}\right)
$$

where we substituted Eq. 112 into the definition of $\langle\mathcal{A}\rangle$. Therefore, evaluating $\langle\mathcal{A}\rangle$ requires only addition of a new step to the sampling procedure from Section 4.1 before Step (1): first, the location $\mathbf{x}_{0}$ is uniformly sampled across the reaction domain $\mathcal{V}$ (as in our introductory example); then, the following steps and the weight function are strictly identical to those for evaluation of $\mathcal{A}\left(\mathbf{x}_{0}\right)$ in Section 4.1. Each optical path that is sampled in this way contributes to the specific absorption at a different location $\mathbf{x}_{0}$. With this Monte Carlo algorithm, $\langle\mathcal{A}\rangle$ is estimated easily, with calculation time $t_{<\mathcal{A}\rangle} \simeq t_{\mathcal{A}} \simeq 5 \mathrm{~s}$, without calculating $\mathcal{A}\left(\mathbf{x}_{0}\right)$ for a set of $M$ locations $\mathbf{x}_{0}$ (leading to calculation time $t_{\langle\mathcal{A}>}=M t_{\mathcal{A}}$ ), where $t_{\mathcal{A}}$ is the calculation time for estimating $\mathcal{A}$ at one location (we estimate that $M \simeq 10^{5}$ in the case of $\mathrm{DiCoFluV}$ ). Actually, this algorithm estimates $\left\langle\mathcal{A}>\right.$ without calculating $\mathcal{A}\left(\mathrm{x}_{0}\right)$ at any location $\mathrm{x}_{0}$ : the integration over the reaction volume and integration over the optical paths are simultaneously statistically sampled. This property makes the Monte Carlo method well suited for numerical implementation of our photobioreactor model, which is based on integral formulae with many dimensions (see Section 1).

In contrast, during evaluation of the average production rate $<r_{x}>=\int_{\mathcal{V}} d \mathbf{x}_{0} \frac{1}{V} r_{x}\left(\mathcal{A}\left(\mathbf{x}_{0}\right)\right)$, integration domains are no longer combined linearly:

$$
<r_{x}>=\int_{\mathcal{V}} d \mathbf{x}_{0} \frac{1}{V} r_{x}\left(\int_{\nu_{\min }}^{\nu_{\max }} d \nu \int_{4 \pi} d \boldsymbol{\omega}_{0} \sigma_{a, \nu} L_{\nu}\left(\mathbf{x}_{0},-\boldsymbol{\omega}_{0}\right)\right)
$$

where the local production rate $r_{x}\left(\mathcal{A}\left(\mathbf{x}_{0}\right)\right)$ is a nonlinear function of $\mathcal{A}\left(\mathbf{x}_{0}\right)$. Because the coupling law $r_{x}(\mathcal{A})$ is nonlinear, it leads to well-known difficulties [83, 84]. Practically, this means that construction of a Monte Carlo algorithm for evaluating $\left\langle r_{x}\right\rangle$ is much more subtle than in the case of $\langle\mathcal{A}\rangle$. The same difficulty is encountered during solution of Schiff's approximation in Section 2 for calculation of the radiative properties of photosynthetic microbial cells. The Monte Carlo code that is used in Section 2 is based on a method presented in [51] that allows for analysis of quadratic functions. In [69], this method is extended to formulation of any analytic ${ }^{11}$ nonlinear function and is successfully implemented for solution of Eq. 115. The resulting Monte Carlo algorithm is used in Section 5.7 (see Fig. 29) for estimation of

\footnotetext{
${ }^{11}$ Here we mean continuous and infinitely differentiable functions.
} 
the production rate $\left\langle r_{x}\right\rangle$ of a DiCoFluV photobioreactor (cultivating $C$. reinhardtii), including rigorous solution of the radiative transfer equation for the spectral radiative properties obtained in Section 2 . The sampling procedure of this algorithm consists of, first, uniform sampling of a location $\left(\mathbf{x}_{0}\right)$ within the reaction volume and then, sampling of a few optical paths starting from this location, according to the optical-pathsampling procedure presented in Section 4.1. Altogether, for estimation with $1 \%$ accuracy, we observed calculation time ranging from 1 to 6 min with the processor Intel Core i7-2720QM 2.20 GHz, depending on the biomass concentration under study (see Fig. 29). This algorithm retains all the features of the Monte Carlo method: the numerical error is systematically evaluated as in Eq. 111; addressing integrals of $<r_{x}>$ is conceptually straightforward (in terms of, for example, annual averages); and the theoretical framework of recent methodological advances such as the zero-variance concept (see [79, 80]) and sensitivity estimation (see Section 4.4) remains accessible. The present study on the capabilities of this novel methodology yielded promising results, in particular for solar-energy applications; however, formulation of coupling laws with discontinuity phenomena, such as the law derived for cyanobacteria in Section 5, remains an open question.

\subsection{Sensitivity analysis}

When a Monte Carlo algorithm is used for estimation of any physical quantity $(B)$, a simple and fast additional procedure can be implemented that simultaneously estimates sensitivity of $B$ to any parameter [79]. This practically means that when Monte Carlo code is available that computes $B$, only a few additional lines of code are needed so that partial derivatives of $B$ are also computed with respect to all the parameters of interest. We are interested either in physical analysis (how does $B$ evolve when a parameter is modified ?) or in optimal design (what is the optimal value of the parameter for a target value of $B$ ?). A general overview of sensitivity estimation is available in [79]. This methodology was implemented in [80, 79] to evaluate sensitivity of the radiation field within a DiCoFluV photobioreactor (see Fig. 26) to radiative properties of microorganisms, to biomass concentration, and to the reflectivity of the optical fibers. In Section 5.7, the same methodology was applied in a straightforward manner to the algorithm from Section 4.3 in order to evaluate sensitivity of the production rate $\left\langle r_{x}>\right.$ of a DiCoFluV photobioreactor to the absorption cross-section, to biomass concentration, and to reflectivity of the optical fibers.

Sensitivity estimation is a methodological advance that we consider mature enough for immediate use in a photobioreactor study. In particular, this approach can be used to accelerate optimization procedures by providing simultaneously $\left\langle r_{x}\right\rangle$ and its gradient in the parameter space, in relation, for example, to the method of steepest descent. Nevertheless, the specific case of sensitivity to parameters defining the geometric structure of the system leads to well-known difficulties that were characterized in [85]. To date, practical implementation of such sensitivity to geometric parameters has been restricted to academic configurations. 


\section{Stoichiometric, thermokinetic, or energetic coupling with a radiation field. Calculation of the main averaged rates and efficiency for the photobioreactor}

As explained in Introduction, the mean volumetric biomass growth rate $\left\langle r_{x}\right\rangle$, which is linked to the stoichiometric equation for the biomass synthesis, is the key process variable appearing in all the other observable variables representative of photobioreactor performance (for instance, the surface growth rate and thermodynamic efficiency). The model then requires formulation of the thermokinetic coupling between the local rate of photon absorption and the local rate of biomass growth, as it is done in the engineering community for any other type of photoreactions [86, 87, 3, 88]. Here, the word "thermokinetic" means that it is impossible to obtain purely kinetic coupling for a light-matter interaction process and for conversion of radiant light energy into biomass through definition of quantum or energetic yields. As already discussed (see Section 1), because photosynthesis shows non-linear behavior $(\langle J(\mathbf{x})\rangle$ is a non-linear function of $\mathcal{A}(\mathbf{x})$ and $\langle f(\mathcal{A}(\mathbf{x}))>$ ), this coupling must be formulated at the local scale (the medium is considered a continuum) before averaging the resulting rates across the total volume of the photobioreactor. As an important consequence, any attempt to formulate direct coupling of a mean spatial growth rate using a given averaged radiative quantity will lead only to representative model formulation. Application of the latter is strongly limited to geometric structures, the range of process variables, and experimental conditions used to identify the model's parameters.

Obtaining a predictive and generic knowledge model of the growth rate in a photobioreactor requires then (i) first to clearly define the main controlling steps involved (and their corresponding yields) in the coupling with the radiation field and (ii) to calculate $a b$ initio all the resulting parameters appearing in this formulation (the reification procedure reduces the parametric space of the model). In this section, we intend to demonstrate that it is possible to formulate such a predictive model of thermokinetic coupling in the limited domain of photosynthesis modeling only, i.e., ignoring the effect of respiration on the global metabolism of the photosynthetic microorganisms. This assumption is not restrictive in the case of prokaryotic cyanobacteria, in which respiration is indeed inhibited by light [89] as soon as it has irradiance levels in the photobioreactor that are at least fivefold higher than the compensation point. This assumption, however, must be considered only as a chloroplast level coupling model for eukaryotic microalgae (see the last part of this section for perspectives on the coupling radiation field and rates for microalgae). In the text below, it will become clear to the reader that such predictive and knowledge coupling formulation relies on a sound and accurate description of the radiation field, thus explaining the special attention paid to this subject matter in the previous sections of this chapter. Finally, the link between rates and stoichiometry will be explicitly determined via the crucial role played by the well-known $\mathrm{P} / 2 \mathrm{e}^{-}$ratio (see Section 5.2). At this stage only, the analysis requiring to deal with a given microorganism will be restricted to the case of $A$. platensis, for which the authors have accumulated a considerable amount of experimental data.

\subsection{Specific rates and thermokinetic coupling with radiation field formulation}

As already explained (see Section 1), local thermokinetic coupling with the radiation field must be formulated at the microorganism scale requiring us to work with molar specific rates $J_{i}$ (in $\mathrm{mol}_{i} \cdot \mathrm{kg}_{x}^{-1} \cdot \mathrm{s}^{-1}$ ), which are defined (as a generalization of Eq. 2) for any compound $i$ from the local molar volumetric rate $r_{i}$ by

$$
J_{i}=\frac{r_{i}}{C_{x}}
$$

In particular, if the specific rate for biomass growth $J_{x}$ is specified in the model, then any molar volumetric rate $r_{i}$ must be deduced from the data on the dry-mass concentration $C_{x}$ and the stoichiometric coefficients involved in the associated stoichiometric equation for biomass synthesis (requiring nevertheless predictive formulation giving a C-molar formula of the produced biomass). The mass volumetric rates are then easily deduced using the molar mass of the compound $i$ being considered. Particularly, the volumetric biomass growth rate $R_{x}$ (in kilograms of dry mass per unit of volume and per unit of time) is given by the following relation:

$$
R_{x}=J_{x} C_{x} M_{x}
$$


where $M_{x}$ is C-molar mass of the biomass produced in the photobioreactor (which will be shown later to depend on the $\mathrm{P} / 2 \mathrm{e}^{-}$ratio, i.e., on the radiation field inside the photobioreactor).

On the other hand, the specific local photon absorption rate (in $\left.\mu \mathrm{mol}_{h \nu} \cdot k g_{x}^{-1} \cdot s^{-1}\right) \mathcal{A}(\mathbf{x})=\int_{P A R} \sigma_{a, \lambda} G_{\lambda}(\mathbf{x}) d \lambda$ is obtained from the radiative approaches described in Sections 3 to 4 above by an integral over all the wavelengths under study (in PAR). Finally, the thermokinetic coupling between kinetic rates and radiant energy absorption rates can be easily formulated from the definition, as for any photo-reactive process [88, 90, 6, 2], of the overall quantum yield $\Phi$ as follows:

$$
J_{x}(\mathbf{x})=\Phi(\mathbf{x}) \mathcal{A}(\mathbf{x})=\rho(\mathbf{x}) \bar{\phi}_{x} \mathcal{A}(\mathbf{x})
$$

First, this equation clearly establishes, as explained in Introduction, that the coupling law inside the photobioreactor is a non-linear local law (depending on the location $\mathbf{x}$ ), which will be shown to be in the form of Eq. 4). The above equation also shows that proper analysis of coupling with radiant energy absorption rates is not compatible with the use of the classical "local growth rate" $\mu$ as a time constant. Second, one should always keep in mind that the overall quantum yield $\Phi$ has been split in two kinds of yield. The purely energetic yield $\rho$, which is a local parameter, takes into consideration all the dissipative phenomena in the light-to-chemical energy conversion processes by the primary mechanisms of the photosynthesis (oxygen evolving complex OEC, reaction centers in photosystems). In contrast, the "stoichiometric" quantum yield $\bar{\phi}_{x}$ is associated with the conservative photon-to-electron mechanisms involved in the Z-scheme for synthesis of ATP and $\mathrm{NADPH}_{2}$. We will see later that this is a time-averaged parameter, i.e., in the linear domain assumption, $\bar{\phi}_{x}$ depends on a spatially averaged function of the radiation field (see Eq. 3).

The thermokinetic coupling (Eq. 118) must be applied directly in the case of eukaryotic microalgae in the limited situation of photosynthesis modeling in chloroplasts (respiration in mitochondria requires an additional part for the coupling model). In case of prokaryotic cyanobacteria, which have common electron carrier chains [89], respiration is inhibited by light, and this law is applicable to the whole-cell metabolism above the compensation point of photosynthesis (corresponding to the specific absorption rate $\mathcal{A}_{c}$ ), i.e., in the form $[6,2]$ :

$$
J_{x}(\mathbf{x})=\rho(\mathbf{x}) \bar{\phi}_{x} \mathcal{A}(\mathbf{x}) H\left(\mathcal{A}-\mathcal{A}_{c}\right)
$$

where the Heaviside function $H\left(\mathcal{A}-\mathcal{A}_{c}\right)$ is introduced $\left(J_{x}=0\right.$ if $\left.\mathcal{A}<\mathcal{A}_{c}\right)$.

\subsection{Structured stoichiometry, biomass composition, and the $P / 2 e^{-}$ratio}

As discussed above, the kinetic rate for biomass must be linked to a stoichiometric equation of biomass growth to enable calculation of the rates of all the abiotic compounds involved in the metabolism of the microorganism. In photosynthesis, this stoichiometric equation can be formulated without any degree of freedom as soon as the mean C-molar formula for the microorganism is known. It was established a long time ago [5] that the biomass composition of a given photosynthetic microorganism depends on the radiation field inside the photobioreactor, regardless of any mineral or carbon source limitations. We will demonstrate in the next part of this section that indeed, the biomass composition is fixed by the well-known $\mathrm{P} / 2 \mathrm{e}^{-}$ratio (the ratio of the mean specific rate of photo-phosphorylation to the mean specific rate of cofactor reduction, see Eq. 120) in the Z-scheme of photosynthesis (see Fig. 28). This ratio is itself a function of the radiation field controlling subsequently quality of the biomass produced in the photobioreactor:

$$
P / 2 e^{-}=P_{2 e}=\frac{\bar{J}_{A T P}}{\bar{J}_{C O F}}=<f(\mathcal{A})>
$$

In the case of $A$. platensis considered here as a model organism of cyanobacteria, it is well known [5, 91] that the $\mathrm{P} / 2 \mathrm{e}^{-}$ratio deviations are balanced by the synthesis of an exopolysaccharide (EPS). Thus, the only degree of freedom for the microorganism's metabolism (corresponding to the value of the $\mathrm{P} / 2 \mathrm{e}^{-}$ratio imposed by the radiation field on the Z-scheme of photosynthesis) is filled by the corresponding ratio of a specific rate of synthesis (of "active biomass" with constant composition) to the specific EPS synthesis rate [5]. The C-molar formulas of active biomass (as the averaged sum of carbohydrates, proteins, lipids, and nucleic acids [classes of macromolecules]) and EPS for A. platensis together with their structured stoichiometry [92] have 
been reported elsewhere [5]. In this section, we provide the resulting structured stoichiometric equation for the total biomass synthesis (active biomass plus EPS) averaged by their respective molar fractions appearing then as a function of the ratio $\mathrm{P} / 2 \mathrm{e}^{-}$:

$$
\begin{gathered}
\mathrm{CO}_{2}+\left(1.806 P_{2 e}-0.885\right) \mathrm{H}_{2} \mathrm{O}+\left(0.507-0.256 P_{2 e}\right) \mathrm{HNO}_{3}+\left(0.013 \mathrm{P}_{2 e}-0.011\right) \mathrm{H}_{2} \mathrm{SO}_{4} \\
+\left(4.475-1.291 P_{2 e}\right) N A D P H, H^{+}+\left(3.146+0.330 P_{2 e}\right) A T P \\
\stackrel{J_{x}}{\longrightarrow} \\
\mathrm{CH}_{\left(1.428+0.112 P_{2 e}\right)} \mathrm{O}_{\left(0.727 P_{2 e}-0.489\right)} N_{\left(0.507-0.256 P_{2 e}\right)} S_{\left(0.013 P_{2 e}-0.011\right)} P_{\left(0.016-0.008 P_{2 e}\right)} \\
+\left(3.130+0.338 P_{2 e}\right) P i+\left(4.475-1.291 P_{2 e}\right) N A D P^{+}+\left(3.146+0.330 P_{2 e}\right) A D P
\end{gathered}
$$

The associated couple of structured stoichiometric equations for photosynthesis (Z-scheme) is then expressed as

$$
\begin{gathered}
\left(4.475-1.291 P_{2 e}\right) N A D P^{+}+\left(4.475-1.291 P_{2 e}\right) H_{2} O \\
\stackrel{J_{C O F}}{\longrightarrow} \\
\left(4.475-1.291 P_{2 e}\right) N A D P H, H^{+}+\left(2.238-0.645 P_{2 e}\right) O_{2} \\
* * * \\
\left(3.146+0.330 P_{2 e}\right)[A D P+P i] \\
\stackrel{J_{A T P}}{\longrightarrow} \\
\left(3.146+0.330 P_{2 e}\right) A T P+\left(3.146+0.330 P_{2 e}\right) H_{2} O
\end{gathered}
$$

Of course, summing Eq. 121 and 122 enables us to obtain the following unstructured equation of biomass synthesis:

$$
\begin{gathered}
\mathrm{CO}_{2}+\left(0.185 \mathrm{P}_{2 e}+0.445\right) \mathrm{H}_{2} \mathrm{O}+\left(0.507-0.256 P_{2 e}\right) \mathrm{HNO}_{3} \\
+\left(0.013 P_{2 e}-0.011\right) \mathrm{H}_{2} \mathrm{SO}_{4}+\left(0.016-0.008 P_{2 e}\right) P i \\
\stackrel{J_{x}}{\longrightarrow} \\
+\left(2.238-0.645 P_{\left(1.428+0.112 P_{2 e}\right)} \mathrm{O}_{\left(0.727 P_{2 e}-0.489\right)} \mathrm{N}_{\left(0.507-0.256 P_{2 e}\right)} S_{\left(0.013 P_{2 e}-0.011\right)} P_{\left(0.016-0.008 P_{2 e}\right)}\right. \\
+(23)
\end{gathered}
$$

It must be noted that these stoichiometric equations have been established with the preferred nitrate $\mathrm{NO}_{3}^{-}$ ion as an $\mathrm{N}$ source, leading to the value of the stoichiometric coefficient:

$$
v_{N A D P H, H^{+}-X}=4.475-1.291 P_{2 e}
$$

If ammonia $\mathrm{NH}_{4}^{+}$is used as the $\mathrm{N}$ source, the same analysis leads to the following stoichiometry:

$$
\begin{gathered}
\mathrm{CO}_{2}+(0.439 \mathrm{P} / 2 e-0.059) \mathrm{H}_{2} \mathrm{O}+(0.507-0.256 \mathrm{P} / 2 e) \mathrm{NH}_{3} \\
+\left(0.013 \mathrm{P}_{2 e}-0.011\right) \mathrm{H}_{2} \mathrm{SO}_{4}+\left(0.016-0.008 P_{2 e}\right) P i \\
\stackrel{J_{x}}{\longrightarrow} \\
\mathrm{CH}_{\left(1.428+0.112 P_{2 e}\right)} \mathrm{O}_{\left(0.727 P_{2 e}-0.489\right)} N_{\left(0.507-0.256 P_{2 e}\right)} S_{\left(0.013 P_{2 e}-0.011\right)} P_{\left(0.016-0.008 P_{2 e}\right)} \\
+\left(1.225-0.134 P_{2 e}\right) \mathrm{O}_{2}
\end{gathered}
$$


in which the stoichiometric coefficient $v_{N A D P H, H^{+}-X}=2 v_{O_{2}-X}$ is sharply different:

$$
v_{N A D P H, H^{+-} X}=2.450-0.268 P_{2 e}
$$

At this stage, we must make the following important observation regarding the Z-scheme for photosynthesis (summarizing all the primary biochemical reactions of the metabolism, i.e., the light reactions of photosynthesis). If we consider only the "stoichiometric" photons involved in this scheme, then we can simply define the mean quantum yield $\bar{\phi}_{x}$ using the data on the stoichiometric coefficient $v_{h v-X}$, directly linked by the structured equations (e.g., Eq. 121) to the stoichiometric coefficient $v_{N A D P H, H^{+}-X}$ and to the value of the $\mathrm{P} / 2 \mathrm{e}^{-}$ratio from

$$
\bar{\phi}_{x}=\frac{10^{-6}}{v_{h v-X}}=\frac{10^{-6}}{2 v_{N A D P H, H^{+}-X}\left(1+P_{2 e}\right)} \quad\left(C-m_{0} l_{x} \cdot \mu m o l_{h \nu}^{-1}\right)
$$

This result is highly important because it means that (i) if we can develop a theory to determine the value of the $\mathrm{P} / 2 \mathrm{e}^{-}$ratio for any situation regarding the radiation field in the photobioreactor, we will then be able to obtain as a predictive mean both the composition of the produced biomass (in terms of the molar fraction of each intracellular-macromolecule class and in terms of the global C-molar formula) and the value of the mean quantum yield $\bar{\phi}_{x}$ involved in the thermokinetic law of coupling. (ii) All the dark reactions of the anabolism in the cells operate under the physical constraint of radiant light transfer controlled only by a tenth of light reactions in the Z-scheme. The first tentative attempt to calculate the $\mathrm{P} / 2 \mathrm{e}^{-}$ratio in a predictive manner [using linear thermodynamics of irreversible processes (LTIP) to formulate a phenomenological model of the Z-scheme] will be presented later, in Section 5.4.

\subsection{Calculation of parameters related to dissipative mechanisms in the light-to-chemical energy conversion process}

Regarding light as a wave or as photons, if we are reasoning either at the microscopic scale (reversibility) or at the macroscopic scale (irreversibility), then we can develop many theoretical approaches to analysis of the light-matter interaction and energy conversion. All these approaches are more-or-less tractable and could be used eventually to calculate the energy dissipation in the process of photon-exciton-electron generation at the early primary stages of photosynthesis. The authors of this chapter are currently working on reconciliation of different approaches in the fields of natural and artificial photosynthesis using materials or molecular complexes as photocatalysts. This reconciliation is necessary to better understand the link between the kinetic approach (flux of photons) and thermodynamic approach (energy flux) in formulation of the coupling. As a whole, this is a considerable amount of work, which is clearly beyond the scope of this chapter, especially because any theory would lead to the same value, with calculation of at least the maximum free chemical energy that can be extracted from a photon interacting with an exciton in a photosynthetic antenna.

We present below a classical approach (based on the macroscopic theory of radiant energy conversion) to such an analysis, which was recently reconciled with the non-equilibrium thermodynamic approach built on the definition of the chemical potential of a photon [93].

The energetic yield $\rho$ represents the dissipative part of the photonic energy absorbed by the antenna, i.e., the part of the absorbed energy that does not lead to electron transfers in the carrier chain and to the reduction of $\mathrm{NADP}^{+}$(energy losses in the light-matter interaction process). First, generally speaking and as explained elsewhere [6], this local value decreases with the local specific absorption rate $\mathcal{A}$ according to a convenient relation postulated here as an approximation of a theoretical quantum mechanical study on the excitation transfer mechanisms in antennas [94]:

$$
\rho \simeq \rho_{M} \frac{1}{1+\frac{\mathcal{A}}{K}}=\rho_{M} \frac{K}{K+\mathcal{A}}
$$

where $\rho_{M}$ is the maximum value of the yield, obtained when the system operates under the optimal thermodynamic conditions, at a very low rate, near the compensation point of photosynthesis (the photon 
absorption rate $\mathcal{A}_{c}$ becomes negligible in regards to the half-saturation constant $\left.K\right)$. It must be pointed out at this stage that even if Eq. 128 remains a representation model, it adds some theoretical background to the well-known hyperbolic behavior of photosynthesis in relation to irradiance values or specific photon absorption rates (with respect to the use of $\rho$ in the coupling law Eq. 118). Second, the maximum energetic yield $\rho_{M}$ will be further discussed in the text below. This yield corresponds to the maximal thermodynamic efficiency (the minimal losses), for the photon conversion in terms of free chemical energy (excitons and electrons in photosystems) at the level of the reaction centers. This maximal efficiency for any radiant-energy conversion process has been extensively debated in the past and eventually clarified by Bejan [95, 96]. We nevertheless propose here to use the simpler approach proposed by Jeter (used in photosynthesis analysis for a long time [97]). These authors simply consider an extension of the Carnot formula for maximal radiation conversion [95], in the same form as that recently obtained independently from the thermodynamic definition of the chemical potential of photons [93]:

$$
\rho_{M}=1-\frac{T}{T_{R}}
$$

where the temperature $T_{R}$ for the radiation is expressed as the ideal blackbody formula of Planck, with assignment to the spectral intensity $L_{c, \bar{\lambda}}$ (under optimal thermodynamic conditions) of the value at the compensation point for photosynthesis in the photobioreactor [6].

Evaluation of $\rho_{M}$ by this approach in a wide range of radiation characteristics (such as angular distribution, frequency, or the value of the intensity) in prokaryotic and eukaryotic microorganisms (they have different optimal temperatures for functioning) generally yields numerical values ranging between 0.76 and 0.82 (in PAR). This result allows us to use in the first approximation the constant value $\rho_{M}=0.8$ with less than $10 \%$ deviation for any photosynthetic microorganism considered in this study. It should be mentioned that this value is in good agreement with the theoretical values determined by a thorough analysis of the excitation transport in an antenna at the quantum mechanical level [94], demonstrating that near the compensation point, photosynthesis operates close to the optimal thermodynamic conditions.

The last kinetic parameter in Eq. 128, the half-saturation constant $K$, must be discussed at this point. Because Eq. 128 is only an approximate relation, today, there is no way to devise a predictive method producing a theoretical value of $K$ as a function of a knowledge description of the OEC functioning. Accordingly, in the model, $K$ appears to be the only parameter that needs to be identified, and this value is indeed specific for a given microorganism. It may be easily obtained, for example, by independent measurements of $\mathrm{O}_{2}$ evolution as a function of the specific photon absorption rate (if the latter is rigorously quantified). Surprisingly, such experiments on different kinds of photosynthetic microorganisms (prokaryotic or eukaryotic) yield rather close values of $K$, within a range of $\pm 30 \%$. Such variation indicates, however, that experimental determination in each microorganism under study can significantly increase the accuracy of the proposed approach. The corresponding value determined for A. platensis and used in this study has already been widely reported by the authors when they wrote the coupling kinetic formula as a function of irradiance $[5,6]$. The corresponding value in specific absorption rate units (see Eqs. 118 and 128) is

$$
K=(1 \pm 0.1) \times 10^{4} \mu m o l_{h \nu} \cdot k g_{x}^{-1} \cdot s^{-1}
$$

5.4. The use of linear thermodynamics of irreversible processes (LTIP) for calculation of parameters related to conservative mechanisms in the process of light-to-chemical energy conversion. P/2 $e^{-}$calculation and analysis

After the pioneering work of Prigogine [12, 98], the use of LTIP for modeling of different patterns of biological behavior was strongly debated in the 80 s and 90 s. When analyzing mainly the metabolism, and particularly the Z-scheme for photosynthesis (the light-driven primary metabolic reactions for photosynthetic microorganisms), it is very important to discuss applicability of such a phenomenological approach to situations involving photons and reactions operating far from equilibrium $\left(\left|A_{i}\right|>R T\right)$. First, we have already shown that only the conserved and stoichiometric photons are considered in our model (calculation of the $\mathrm{P} / 2 \mathrm{e}^{-}$ratio and stoichiometric quantum yield $\bar{\phi}_{x}$ ), and this approach enables proper use of the linear energy converter formalism. Second, it was demonstrated in the $80 \mathrm{~s}$ [13, 14, 99] that the mean variables describing biological systems and satisfying the nonasymptotic stability criterion (in the sense of Lyapunov) 
obey linear phenomenological relations. This state of affairs requires of course choosing a characteristic time point to perform time-averaged integrals for these variables and working after that with mean rates as multi-linear functions of mean affinity levels obeying the reciprocity Onsager relations. Consequently, LTIP seems to plausibly describe the mean functioning of processes far from equilibrium: unstable for instantaneous values but stable for time-averaged values during operation in a highly organized spatial biological structure [14]. The characteristic time point under consideration for variable observations must be consequently chosen at a level enabling the use of the pseudo-steady-state assumption for intermediate products of the metabolism (approximately one minute). This period is clearly of the same order of magnitude as the mixing time inside a photobioreactor; consequently, we will assume below that because all the relations involved are linear, the mean time variables $\bar{B}$ are equivalent to the spatially averaged values $\langle B\rangle$ inside the photobioreactor. Eventually, there are no additional restrictions on the use of LTIP to describe the Z-scheme for photosynthesis in the same form as it has been done in the seminal work of Stucki [99, 100] and Dussap [14] for respiration.

As for the previous conditions of applicability, it is then possible to use linear phenomenological thermodynamics of irreversible processes to analyze the coupling between redox reactions leading to reducing $\mathrm{NADPH}_{2}$ synthesis (specific molar rate $J_{C O F}$ ) and the photo-phosphorylation mechanisms leading to ATP synthesis (specific molar rate $J_{A T P}$ ) according to the Z-scheme of photosynthesis (Fig. 28). The ratio of these two mean specific rates is defined as the $\mathrm{P} / 2 \mathrm{e}^{-}$ratio (see Eq. 120):

$$
P_{2 e}=\frac{\bar{J}_{A T P}}{\bar{J}_{C O F}}
$$

Because we work with "stoichiometric" photons (corresponding to the transfer of one electron), we can define the Z-scheme as two coupled reactions involving four photons for one molecule of water split at the OEC of photosystem II. This model requires that one equation be exergonic (positive affinity) and one endergonic (negative affinity), so that the partition among the four photons is unique and straightforward [5]:

$$
\begin{aligned}
& 3 h \nu+A D P+P i \quad \stackrel{\bar{J}_{A T P}}{\longrightarrow} A T P+H_{2} O \quad \text { where } \bar{A}_{A T P}>0 \\
& \sum^{2}(0)^{3} \\
& h \nu+H_{2} O+N A D P^{+} \quad \stackrel{\bar{J}_{C O F}}{\longrightarrow} \quad \frac{1}{2} O_{2}+N A D P H, H^{+} \quad \text { where } \bar{A}_{C O F}<0
\end{aligned}
$$

It must be noted here that this formulation remains purely phenomenological and must not be used in any case as a tentative mechanistic explanation of cyclic photo-phosphorylation. The affinity values $A_{i}$ in this formula are defined by means of the chemical potential $\tilde{\mu}_{i}$ calculated under cytosolic conditions from

$$
\begin{aligned}
& \bar{A}_{A T P}=3 h \nu+\tilde{\mu}_{A D P}+\tilde{\mu}_{P i}-\tilde{\mu}_{A T P}-\tilde{\mu}_{H_{2} O} \\
& \bar{A}_{C O F}=h \nu+\tilde{\mu}_{H_{2} O}+\tilde{\mu}_{N A D P^{+}}-\tilde{\mu}_{N A D P H}-\tilde{\mu}_{H^{+}}-\frac{1}{2} \tilde{\mu}_{O_{2}}
\end{aligned}
$$

Assuming that one mole of photons at $\lambda=680 \mathrm{~nm}$ corresponds to enthalpy of $176 \mathrm{~kJ} / \mathrm{mol}$ and using the models of calculation of thermodynamic properties developed in our lab [101], we obtain the following result for the theoretical affinity values:

$$
\begin{aligned}
& \bar{A}_{A T P, \max }=3 h \nu_{\max }-32,5=495,2{\mathrm{~kJ} . \mathrm{mol}^{-1}} \\
& \bar{A}_{C O F, \max }=h \nu_{\max }-216,3=-40,4{\mathrm{~kJ} . \mathrm{mol}^{-1}}^{-1}
\end{aligned}
$$

These affinity values are considered maximal because they are calculated with the enthalpy of a photon $(h \nu)$, but the optimization procedure that is explained below will result in new affinity values at lower free 


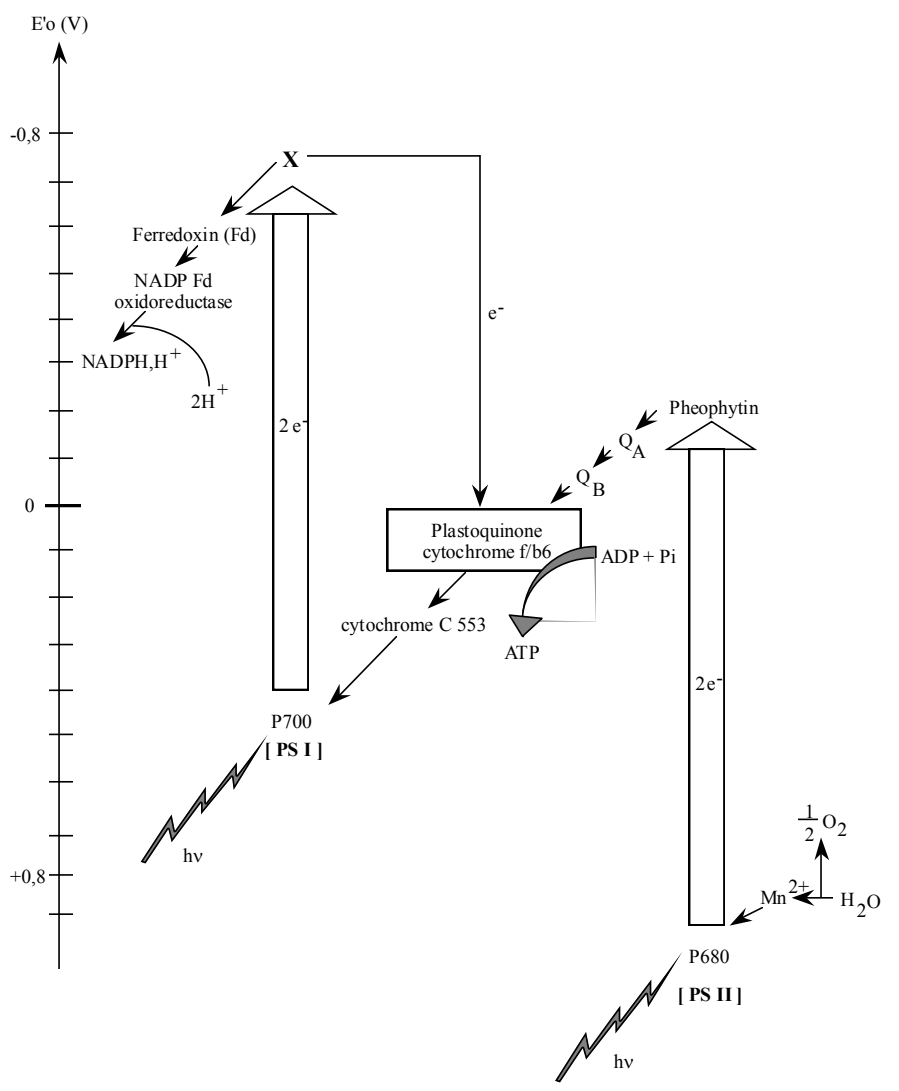

Figure 28: The Z-scheme of photosynthesis for cyanobacteria (from [5]). The cyclic photo-phosphorylation pathway enabling values of $\mathrm{P} / 2 \mathrm{e}^{-}$greater than 1.0 is indicated.

enthalpy $h \nu_{\text {eff }}$. Therefore, it will become evident that energetic efficiency of the coupling decreases with the increasing specific photon absorption rate $\langle\mathcal{A}\rangle$. By means of the partition of the entropy balance in the photobioreactor [70], it is then possible to derive the expression for the rate of entropy production $\sigma$ (or the dissipation function) in the reactive system under study (the Z-scheme). Assuming here the system as isotherm, we will soundly define the mean specific isotherm dissipation function at a given optimal temperature $\left(T^{*}\right)$ as $\bar{\sigma}^{*}=\bar{\sigma} T^{*} / C_{x}$ in the following form:

$$
\bar{\sigma}^{*}=\sum_{j=1}^{r} \bar{J}_{j} \bar{A}_{j}=\bar{J}_{A T P} \bar{A}_{A T P}+\bar{J}_{C O F} \bar{A}_{C O F} \geq 0
$$

Contrary to the complete expression established previously by the authors for photobioreactors [70], the specific radiant light absorption rate here is ignored in the dissipation function because the radiant light energy in the photochemical process was taken into account directly in the affinity definitions (see Eqs. 131 to 134$)$. 
The theory of linear energy converters then postulates a multi-linear relation between affinity values and specific rates, leading to the following equations, with the help of reciprocity relations of Onsager:

$$
\begin{aligned}
& \bar{J}_{A T P}=L_{A A} \bar{A}_{A T P}+L_{A C} \bar{A}_{C O F} \\
& \bar{J}_{C O F}=L_{A C} \bar{A}_{A T P}+L_{C C} \bar{A}_{C O F}
\end{aligned}
$$

where the phenomenological coefficients $L_{i j}$ must be eliminated from convenient normalization [99, 14] leading to definitions of the coupling coefficient:

$$
q=\frac{L_{A C}}{\sqrt{L_{A A} L_{C C}}}
$$

of the phenomenological stoichiometric coefficient:

$$
\chi=\sqrt{\frac{L_{C C}}{L_{A A}}}
$$

and finally, the ratio of generalized forces:

$$
x=\chi \frac{\bar{A}_{C O F}}{\bar{A}_{A T P}}, \quad x<0
$$

Thus, the specific rates of cofactor or ATP synthesis can be expressed with the help of the previous variables as

$$
\begin{aligned}
& \bar{J}_{A T P}=L_{A A} \bar{A}_{A T P}(1+q x) \\
& \bar{J}_{C O F}=\chi L_{A A} \bar{A}_{A T P}(q+x)
\end{aligned}
$$

yielding the values of chemical power as the key parameters involved in the dissipation function and in the energetic analysis of the photobioreactor (see Section 5.5):

$$
\begin{aligned}
& \bar{J}_{A T P} \bar{A}_{A T P}=L_{A A} \bar{A}_{A T P}^{2}(1+q x) \\
& \bar{J}_{C O F} \bar{A}_{C O F}=L_{A A} \bar{A}_{A T P}^{2} x(q+x)
\end{aligned}
$$

and eventually allowing us to establish a tractable expression for the specific source of entropy production rate:

$$
\bar{\sigma}^{*}=L_{A A} \bar{A}_{A T P}^{2}\left(1+2 q x+x^{2}\right)
$$

The ultimate variable from which we intend to derive all the predictive information on the Z-scheme modeling is the $\mathrm{P} / 2 \mathrm{e}^{-}$ratio rewritten from the normalized variables as

$$
P_{2 e}=\frac{1+q x}{\chi(q+x)}
$$

The whole theoretical approach relies now on the ability to calculate, for any condition of radiation field in the photobioreactor, the variables $q, \chi$, and $x$ in a predictive manner. This calculation can be performed in two steps. First, it can be demonstrated [14] that there are two non-adaptive conditions (i.e., fixed conditions arising at the early stage of a cell's life cycle), allowing us to obtain constant values for $q$ and $\chi$. The first condition links the stoichiometric coefficient for the phosphorylation to the phenomenological stoichiometric coefficient in non-ideal coupling:

$$
\chi=v_{C O F-A T P} q
$$

It is well established that there is only one site for phosphorylation in the electron carrier chain of photosynthesis; therefore, $v_{C O F-A T P}=1$, and consequently, according to Eq. 147:

$$
\chi=q
$$


The second non-adaptive condition means that the optimal thermodynamic functioning of the cell corresponds to maximization of chemical power (Eqs. 143 and 144), leading to calculation of the coefficient $q[99,14]$ :

$$
q=\sqrt{2(\sqrt{2}-1)}=0.91
$$

Second, we must invoke the adaptive conditions of the cells experiencing a given mean radiation field inside the photobioreactor. As explained throughout this chapter, any photosynthetic microorganism operates under conditions of physical limitation by light. This means that the radiation field (or rather a spatial averaged function of the radiation field for the time constants considered in this analysis) is a constraint entirely determining the behavior of the Z-scheme functioning in the cells. If we assume that this behavior obeys the principle of minimum entropy production rate $[5,14,98,99]$, then the ratio $x$ of generalized forces can be determined by an optimization procedure confirming that

$$
d \bar{\sigma}^{*}=0
$$

This classical optimization problem under constraints requires introducing a Lagrange function $\mathcal{L}$ with Lagrange multipliers $\lambda_{k}$ associated with the constraints $g_{k}$ in the following form:

$$
\overline{\mathcal{L}}=\bar{\sigma}^{*}-\sum_{k} \lambda_{k} \bar{g}_{k}
$$

and requires calculating the partial derivatives

$$
\left(\partial_{\bar{A}_{j}} \overline{\mathcal{L}}\right)_{\bar{A}_{k}}=0 \quad, \quad(k \neq j)
$$

Generally speaking, the main difficulty here is to formulate the constraints $g_{k}$ linked to an averaged function of the radiation field $\langle f(\mathcal{A})\rangle$ in all the situations encountered in photobioreactors (e.g., a kinetic regime, luminostat regime, and photo-limitation with dark zones). A comprehensive analysis of this complicated problem is still a work in progress by the authors and clearly beyond the scope of this chapter. In contrast, it is quite easy to examine two extreme situations for which the unique constraint on the Z-scheme functioning may be formulated as a limit, independently of knowledge about the function $\langle f(\mathcal{A})\rangle$.

The first situation that can be envisaged concerns functioning close to the compensation point for photosynthesis (very low specific photon absorption rates $\langle\mathcal{A}\rangle \rightarrow 0$ ) corresponding to the strongest physical limitation by radiant light energy transfer. In this condition, the specific rate $\bar{J}_{A T P}$ is the constraint imposed on the photosynthetic functioning $[14,5]$ and must be assumed to be a constant. Eq. 151 can then be written as

$$
\overline{\mathcal{L}}=L_{A A} \bar{A}_{A T P}^{2}\left(1+2 q x+x^{2}\right)-\lambda L_{A A} \bar{A}_{A T P}(1+q x)
$$

Accordingly, the optimization (Eq. 152) leads to calculation of the ratio of generalized forces:

$$
x=0
$$

With the previous values of $q$ and $\chi$, it is then possible to obtain the $\mathrm{P} / 2 \mathrm{e}^{-}$value in this situation from Eq. 146: $P_{2 e}=1.21$. It is important to note that this value is very close to the value of $P_{2 e}=1.23$ obtained independently for the active biomass of A. platensis in a complete analysis of its metabolism [5] and used in a previous stoichiometric equation for total biomass synthesis: Eq. 121 or 123.

The second simple situation is to consider, at the other extreme, the maximal saturation rate for photosynthesis (very high specific photon absorption rates $\langle\mathcal{A}\rangle \rightarrow \infty$ ), i.e., the functioning without the light transfer limitation. In this case, the constraint imposed on the Z-scheme metabolism is the chemical power output $\bar{J}_{A T P} \bar{A}_{A T P}[5,14]$, which is constant and independent of the radiation field. The Lagrange function takes the following form:

$$
\overline{\mathcal{L}}=L_{A A} \bar{A}_{A T P}^{2}\left(1+2 q x+x^{2}\right)-\lambda L_{A A} \bar{A}_{A T P}^{2}(1+q x)
$$


and the optimization yields

$$
x=\frac{\sqrt{1-q^{2}}-1}{q}=-0,643
$$

with the highest value for the $\mathrm{P} / 2 \mathrm{e}^{-}$ratio: $P_{2 e}=1.71$.

Although general formulation of the problem is beyond the scope of this chapter as explained above, it is nevertheless possible to report here an important conclusion for a photobioreactor operating in optimal situations [7] in terms of its kinetic or energetic performance (luminostat or photo-limitation). In this case, the maximum $\mathrm{P} / 2 \mathrm{e}^{-}$value that can be reached with very high specific photon absorption rates [corresponding to incident photon flux density (PFD) of a full-sun AM 1.5 near $2000 \mu m o l_{h \nu} \cdot m^{-2} \cdot s^{-1}$ ] is $P_{2 e}=1.5$. The highest values of the $\mathrm{P} / 2 \mathrm{e}^{-}$ratio do not occur under natural outdoor sunlight conditions and in most of the artificially illuminated indoor photobioreactors. Considering now this optimal range of the $\mathrm{P} / 2 \mathrm{e}^{-}$ functioning for the Z-scheme in optimal situations for photobioreactor operation, i.e., $1.2<P_{2 e}<1.5$, and using Eqs. 124 and 127 for calculation of the mean stoichiometric quantum yield of the coupling law $\bar{\phi}_{x}$, we obtain a constant value equal to

$$
\bar{\phi}_{x}=7.8 \times 10^{-8} C-\operatorname{mol}_{x} \cdot \mu m o l_{h \nu}^{-1}
$$

This surprising result is caused by the opposite effects in Eq. 127 where an increase in the $\mathrm{P} / 2 \mathrm{e}^{-}$ratio is strictly compensated by a decrease in the stoichiometric coefficient $v_{N A D P H, H^{+}-X}$ in the global stoichiometry for the quantum yield calculation. This phenomenon is important because it shows that the "stoichiometric" coupling may be considered linear coupling in Eqs. 118 and 119, independently of any function $\langle f(\mathcal{A})\rangle$. It also shows that the energetic yield $\rho$ remains the only radiation field-dependent parameter. In contrast, the biomass composition, i.e., stoichiometry of the photosynthetic growth reaction and the C-molar formula of the biomass inside the photobioreactor are clearly dependent on the radiation field and $\mathrm{P} / 2 \mathrm{e}^{-}$.

The value of the quantum yield (Eq. 157) was obtained with the preferred $\mathrm{N}$ source (nitrate) in the stoichiometry, but the calculation could be performed with ammonia as the $\mathrm{N}$ source. In this case, using Eq. 126 instead of Eq. 124 in Eq. 127, we also obtain the quasi-constant value:

$$
\bar{\phi}_{x}=1.0 \times 10^{-7} C-\operatorname{mol}_{x} \cdot \mu m o l_{h \nu}^{-1}
$$

This interesting result shows that the efficiency of photosynthesis is $25 \%$ higher if ammonia is used instead of nitrate as the $\mathrm{N}$ source; this is a well-known phenomenon for cultivation of aerobic microorganisms as mentioned by Roels [92].

\subsection{Thermodynamic efficiency and energetic-coupling analysis}

As discussed in Introduction, the previous thermokinetic model of coupling (Sections 5.1 to 5.4) may be used to predictively calculate the mean averaged specific rates $\left\langle J_{i}\right\rangle$, volumetric rates $\left\langle r_{i}\right\rangle$, or surface rates $\left\langle s_{i}\right\rangle$ by a volume integral for any given geometric structure of a photobioreactor. This approach requires first solving the radiative transfer equation using one-dimension-approximated or rigorous numerical approaches such as the Monte Carlo method discussed in Section 4 or the finite element method (proposed in [70]) used for calculation of the local specific rate of photon absorption $\mathcal{A}$. In all these situations, it is easy to evaluate the local volumetric rate of radiant energy absorbed $\mathcal{A}_{v}$ and its mean averaged integral over the total volume of the photobioreactor $\left\langle\mathcal{A}_{v}\right\rangle$. This parameter is derived from the data on the specific rate of photon absorption $\mathcal{A}$ from

$$
\mathcal{A}_{v}=\mathcal{A} C_{x} \aleph \quad\left(\text { in } W \cdot m^{-3}\right)
$$

where $\aleph$ (in $J \cdot \mu m o l_{h \nu}^{-1}$ ) is a conversion factor between micromoles of photons and joules (depending on the spectral nature of light in the incident PFD as a boundary condition). This is all that we need to calculate thermodynamic efficiency of the photochemical process as a whole. Considering first the thermodynamic 
efficiency of the photosynthesis inside the photobioreactor and using the entropic analysis proposed by Cornet et al. [70], we obtain the following relation:

$$
\left\langle\eta_{t h, \phi S}\right\rangle=\frac{\sum_{j=1}^{r} \sum_{p=1}^{n} v_{p, j}<r_{j}>\tilde{\mu}_{p}}{<\mathcal{A}_{v}>-\sum_{j=1}^{r} \sum_{s=1}^{m} v_{s, j}<r_{j}>\tilde{\mu}_{s}} \simeq \frac{<r_{x}>\Delta g_{x}^{\prime 0}}{<\mathcal{A}_{v}>}
$$

where all the parameters were determined by a predictive approach. Defining the total thermodynamic efficiency now requires working with the mean incident $\mathrm{PFD}<q_{\cap}>$ arriving into the photobioreactor and then with surface rates rather than volumetric rates (see Section 1):

$$
\left\langle\eta_{t h}\right\rangle=\frac{\sum_{j=1}^{r} \sum_{p=1}^{n} v_{p, j}<s_{j}>\tilde{\mu}_{p}}{<\mathcal{A}_{v}>-\sum_{j=1}^{r} \sum_{s=1}^{m} v_{s, j}<s_{j}>\tilde{\mu}_{s}} \simeq \frac{<s_{x}>\Delta g_{x}^{\prime 0}}{<q_{\cap}>}
$$

It has been previously demonstrated in both cases that these thermodynamic-efficiency levels strongly decrease with the increasing incident PFD $q_{\cap}$; this effect forms the basis of the light dilution concept for improvement of thermodynamic performance of photobioreactors [7, 2]. For example, in the $\mathrm{DiCoFluV}$ concept (for which simulations of the complete model are shown in Fig. 29 at the end of this section), the sunlight capture surface can be 50-fold smaller than the light distribution surface inside the photobioreactor (see Chapter 5 by Pruvost et al. in this book).

\subsection{Experimental validation of the proposed model for different simple geometric structures of a photobiore- actor}

Experimental validation of the predictive knowledge model described in Sections 2 to 5 is shown below for simple geometric structures of photobioreactors. These results have already been published for analysis of validity of a simple and reliable engineering equation [6]. They are used here again to analyze validity of the complete model, i.e., for validation of the predictive approach as presented throughout this chapter. These experimental results have been obtained in eight very different, completely stirred photobioreactors, whose working liquid volume varied between 0.1 and $77.0 \mathrm{~L}$, with the dark-volume fraction $f_{d}$ ranging between 0 and 0.48 (i.e., the volume fraction of photobioreactors that is not illuminated by design). Various kinds of geometric structures have been explored with different artificial illumination systems and with different methods of mixing the culture medium. In all the experiments, the microorganism was Arthrospira (Spirulina) platensis PCC 8005 grown axenically on the Cogne medium [102]. The temperature $\left(35-36^{\circ} \mathrm{C}\right)$ and $\mathrm{pH}$ (between 8 and 10) were maintained at the levels optimal for growth. Incident hemispherical PFD ranged between 30 and $1600 \mathrm{\mu mol}_{h \nu} \cdot \mathrm{m}^{-2} \cdot \mathrm{s}^{-1}$ (PAR, with multi-point measurements by means of the LICOR cosine quantum sensor LI-190SA, with subsequent confirmation by actinometry). The main parameters influencing the biomass volumetric growth rates (geometric structure and characteristics of the illumination system), the culture conditions (mixing as well as $\mathrm{pH}$ and temperature control), and the operating conditions (batch mode or continuous culture) were described elsewhere [6] and are summarized here only briefly (see Table 2).

In all the experiments, the experimental mean volumetric growth rates were obtained according to the biomass balance in a well-mixed photobioreactor (defining the so-called residence time for continuous culture as $\left.\tau=\frac{V_{L}}{Q_{L}}\right)$ :

$$
<R_{x}>=\frac{C_{x}}{\tau}+d_{t} C_{x}
$$

The resulting values of $\left\langle R_{x}\right\rangle$, all obtained under optimal conditions of limitation by light (luminostat $\gamma$ $=1$, or photo-limitation $\gamma<1$, see [7]) are presented in Table 2. They are compared with the predictive model calculations presented in this chapter, where the radiative transfer equation was solved using the one-dimensional two-flux approximation for all the simple geometric structures of photobioreactors except for reactor PBR 2 (as indicated in Table 2), for which we used the three-dimensional finite element method developed by Cornet et al. [70]. As shown in the table, the mean deviation between the experimental results and the model calculation is less than $5 \%$ (i.e., within the range of the experimental standard deviation), 
thus confirming the ability of the proposed predictive approach to quantify photobioreactor performance under many conditions of operation.

It must be noted that the new advances in the Monte Carlo method presented above would have led to the same $\left\langle R_{x}\right\rangle$ values for the model calculation, with numerous advantages such as shorter calculation time, assessment of standard deviations, model parameter sensibility analysis, evaluation of any local parameter without calculation of the whole field, possibility of increasing integral dimensionality of the problem without increasing the calculation time, and no need for a specific mesh grid. Additionally, as explained in Section 4, recent advances in this field allow researchers to separate the Monte Carlo algorithm formulation from analysis of complexity of a photobioreactor's geometric structure [79, 80]. These are the reasons why the authors today strongly recommend the Monte Carlo method, at least in the case of complex geometric structure or for development of a reference solution. Such Monte Carlo simulations are implemented in the next section for eukaryotic microalgae.

Finally, the reader will also find experimental validation of the proposed model in other interesting situations in Chapter 5 written by Pruvost et al. in this book. 


\begin{tabular}{|c|c|c|c|c|}
\hline 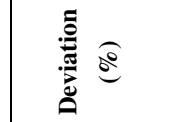 & $\begin{array}{lll}n & n & n \\
+ & + & +\end{array}$ & 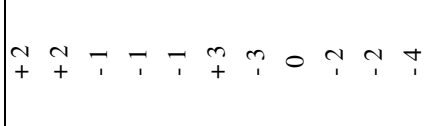 & $\begin{array}{ccc}\infty & \infty & 0 \\
1 & + & +\end{array}$ & $\stackrel{n}{n}+\frac{n}{+}+$ \\
\hline 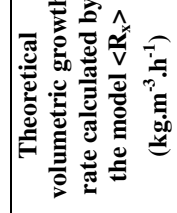 & 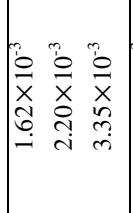 & 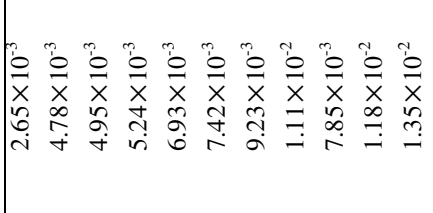 & 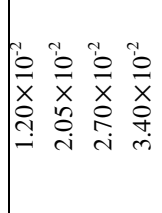 & 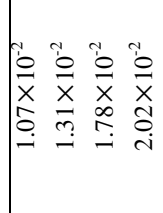 \\
\hline 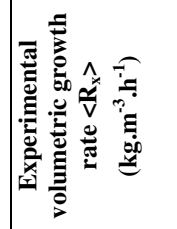 & 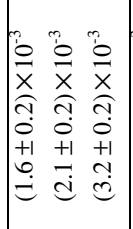 & 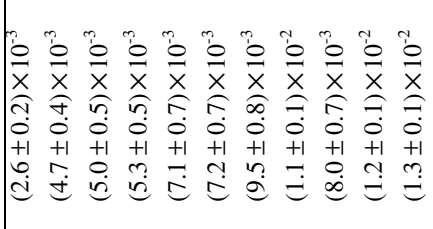 & 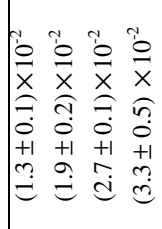 & 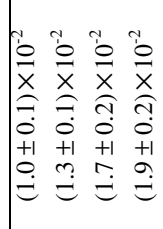 \\
\hline 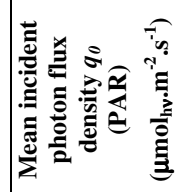 & o in $\infty$ & 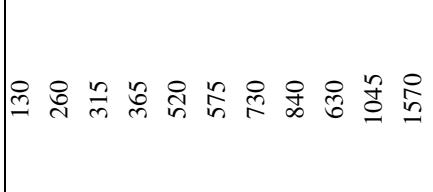 & 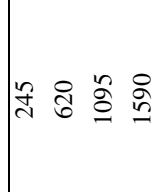 & 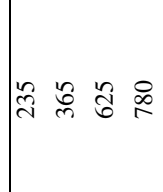 \\
\hline 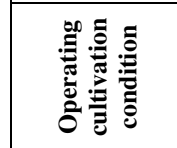 & 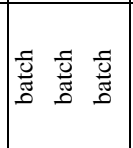 & 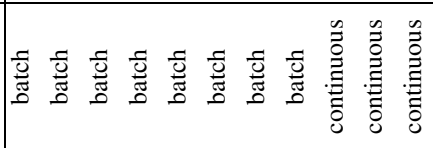 & 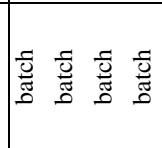 & 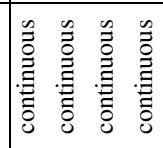 \\
\hline 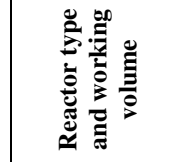 & 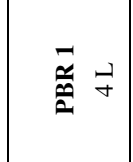 & 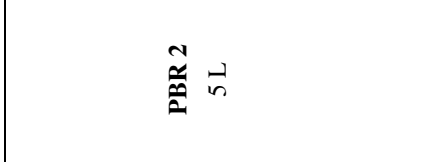 & मे & 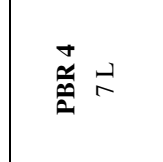 \\
\hline 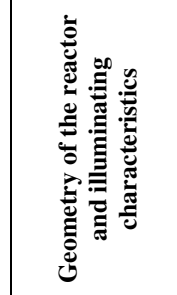 & 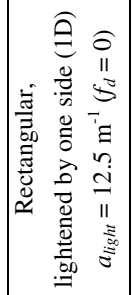 & 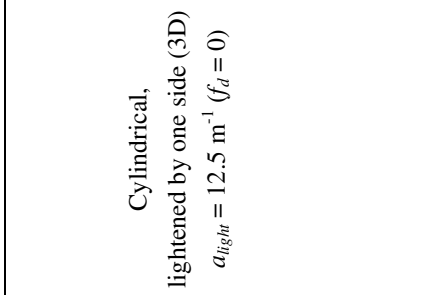 & 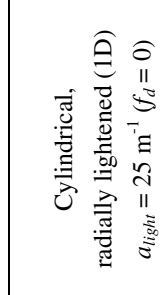 & 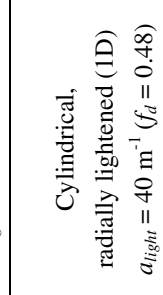 \\
\hline
\end{tabular}




\begin{tabular}{|c|c|c|c|c|c|}
\hline 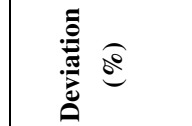 & $\mp$ & $\begin{array}{lll}1 & 0 \\
1 & +\end{array}$ & $\begin{array}{lll}n & m & n \\
1 & 1 & 1\end{array}$ & † & $n$ \\
\hline 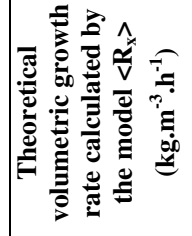 & 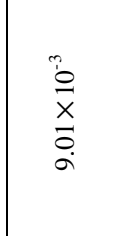 & 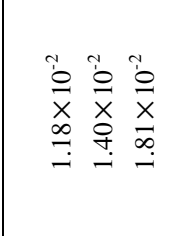 & 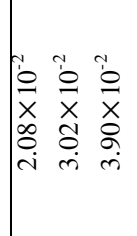 & 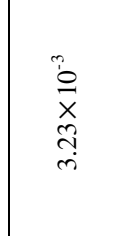 & $\begin{array}{l}3 \\
0 \\
x \\
o \\
0 \\
\end{array}$ \\
\hline 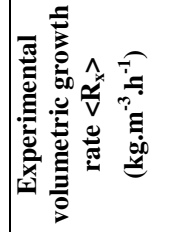 & 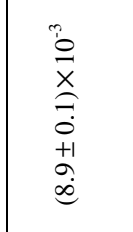 & 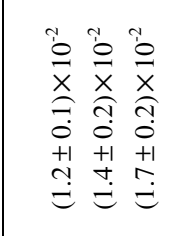 & 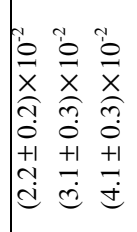 & 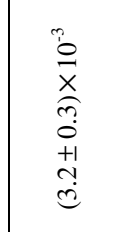 & 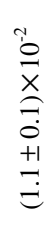 \\
\hline 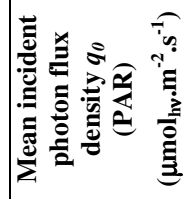 & 8 & 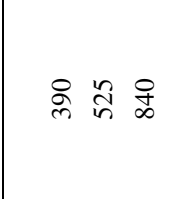 & 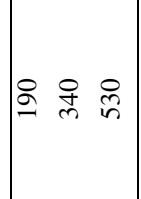 & $m$ & $\stackrel{m}{m}$ \\
\hline 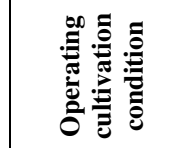 & $\begin{array}{l}\frac{\tilde{0}}{\tilde{U}} \\
\text { פ }\end{array}$ & 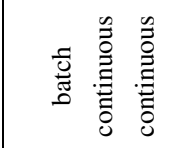 & 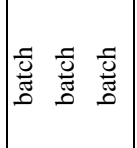 & 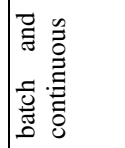 & 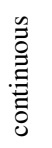 \\
\hline 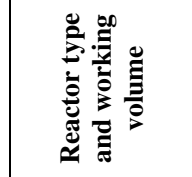 & 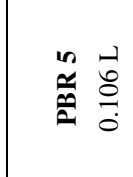 & $\stackrel{b}{\stackrel{0}{*}}$ & له & 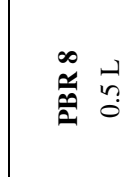 & \\
\hline 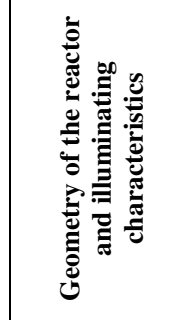 & 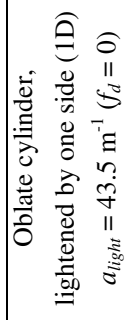 & 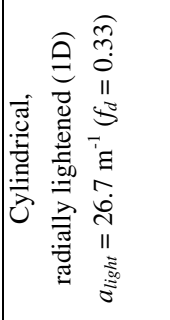 & 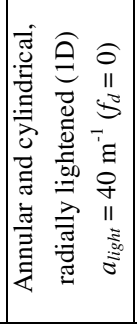 & 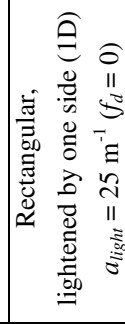 & \\
\hline
\end{tabular}

Table 2: Comparison between experimental biomass volumetric growth rates obtained in different kinds of photobioreactors cultivating Arthrospira platensis and the knowledge model presented in this chapter. The photobioreactors' main characteristics and the experimental conditions are described in [6]. 


\subsection{Perspectives on formulation of thermokinetic coupling for eukaryotic microalgae}

The preliminary work on formulation of the thermokinetic coupling that we just presented above is limited to the metabolism of cyanobacteria or, in the case of eukaryotic microalgae, to their chloroplast functioning. Obviously, substantial additional work is needed to obtain such a general and predictive coupling law for eukaryotic photosynthetic microorganisms containing chloroplasts and mitochondria (which enable respiration at light). Although the LTIP approach that was described above has been strongly improved in relation to mitochondrial function [14, 99, 100], general formulation requires at least research into additional coupling between photosynthesis and respiration (including elucidation of the effect of light on respirationspecific rates). Evidently, this coupling must be formulated at the primary level, i.e., taking into account the commutation between $\mathrm{NADPH}_{2}$ and $\mathrm{NADH}_{2}$ and its re-oxidation in the electron carrier chains of mitochondria. This commutation is possibly constrained by the radiation field inside the photobioreactor and the $\mathrm{P} / \mathrm{O}$ ratio of mitochondria that is properly described by means of LTIP to analyze this new coupling. Unfortunately, we are today unable to provide a knowledge model for this thermokinetic coupling, and here, we will simply report tentative formulation of a representative law that seems consistent with numerous experimental observations (see Chapter 5 by Pruvost et al. in this book).

Because only primary stages are involved in photosynthesis and respiration (electron carrier chains and some key enzymes in the cofactor exchange reactions), it seems convenient to first formulate a kinetic coupling law related to the specific net oxygen production rate $J_{\mathrm{O} 2}$. Assuming that respiration rates are affected by the radiation field (as confirmed for some microalgae and reported for experimental measurements by specific analytical methods [103]), we can propose a rather symmetrical local law for respiration (this law is related to the already developed approach at the chloroplast level in the general form [2]):

$$
J_{O_{2}}(\mathbf{x})=\rho_{M} \frac{K}{K+\mathcal{A}(\mathbf{x})} \bar{\phi}_{O_{2}} \mathcal{A}(\mathbf{x})-J_{r} \frac{K_{r}}{K_{r}+\mathcal{A}(\mathbf{x})} \quad\left(m o l_{O 2} \cdot k g_{x}^{-1} \cdot s^{-1}\right)
$$

where the specific respiration rate is simply related to the rate of cofactor regeneration by respiration:

$$
J_{r}=\frac{J_{N_{A D H}}}{v_{N A D H_{2}-O_{2}}}=\frac{J_{C O F}}{v_{N A D H_{2}-O_{2}}} \text { and } v_{N A D H_{2}-O_{2}}=2
$$

The coupling equation above is still strongly linked to the radiation field, including the respiration term. At obscurity (anywhere in the photobioreactor) or at a very low value of $\mathcal{A}(\mathbf{x})$, the maximal respiration rate $J_{r}$ must be considered constant and can be measured in independent experiments. In this case, the value of the new parameter $K_{r}$ is not independent because it can be deduced directly from the data on the specific photon absorption rate at the compensation point $\mathcal{A}_{c}$ (the other parameters known in our knowledge model):

$$
K_{r}=\frac{\mathcal{A}_{c}}{\frac{J_{N A D H_{2}}}{v_{N A D H_{2}-O_{2}} \rho_{M} \bar{\phi}_{O_{2}}}\left[\frac{1}{\mathcal{A}_{c}}+\frac{1}{K}\right]-1}
$$

In these equations, once $\mathcal{A}_{c}$ is specified, it is possible to use the predictive parameters described in the preceding sections $\left(\rho_{M}\right.$ and $\left.K\right)$. Then, the stoichiometric oxygen quantum yield is easily deduced from the data on the $\mathrm{P} / 2 \mathrm{e}^{-}$ratio in the chloroplast from :

$$
\bar{\phi}_{\mathrm{O}_{2}}=\frac{10^{-6}}{4\left(1+P / 2 \mathrm{e}^{-}\right)} \simeq 1.1 \times 10^{-7} \mathrm{~mol}_{\mathrm{O} 2} \cdot \mu_{\mathrm{mol}}^{-1}
$$

Finally, if necessary, the mean volumetric biomass growth rate in the photobioreactor $\left\langle R_{x}>\right.$ is easily obtained by means of a spatial integral of the specific rate of net oxygen production $<J_{O 2}>$ and again by means of

$$
<R_{x}>=\frac{<J_{\mathrm{O}_{2}}>C_{x} M_{x}}{v_{\mathrm{O}_{2}-x}} \quad\left(k g_{x} \cdot \mathrm{m}^{-3} \cdot \mathrm{s}^{-1}\right)
$$

where $v_{\mathrm{O}_{2}-x}$ and $M_{x}$ are $\mathrm{P} / 2 \mathrm{e}^{-}$-dependent parameters. 
Obviously, further work is necessary to devise a predictive and knowledge model of thermokinetic coupling for eukaryotic microalgae. This model may help to predict the specific compensation absorption rate $\mathcal{A}_{c}$ for any microorganisms (specific data explaining mainly the kinetic diversity of microalgae), which is perhaps linked to some hydrodynamic or physiological parameters. Such a model could also be used for predictive stoichiometric analysis, thus enabling assessment of quality of the produced biomass.

Now, the proposed kinetic coupling law (Eq. 163), already used for example by Takache et al. [72] for $C$. reinhardtii cultivation in a simple rectangular photobioreactor, is discussed in the text below as illustration of the whole methodology developed throughout this chapter, especially the use of the integral Monte Carlo formulation for easy calculation of the radiation field, volumetric biomass growth rate $\left\langle R_{x}\right\rangle$, standard deviation of the results, and all the desired types of sensitivity (with the same calculation time).

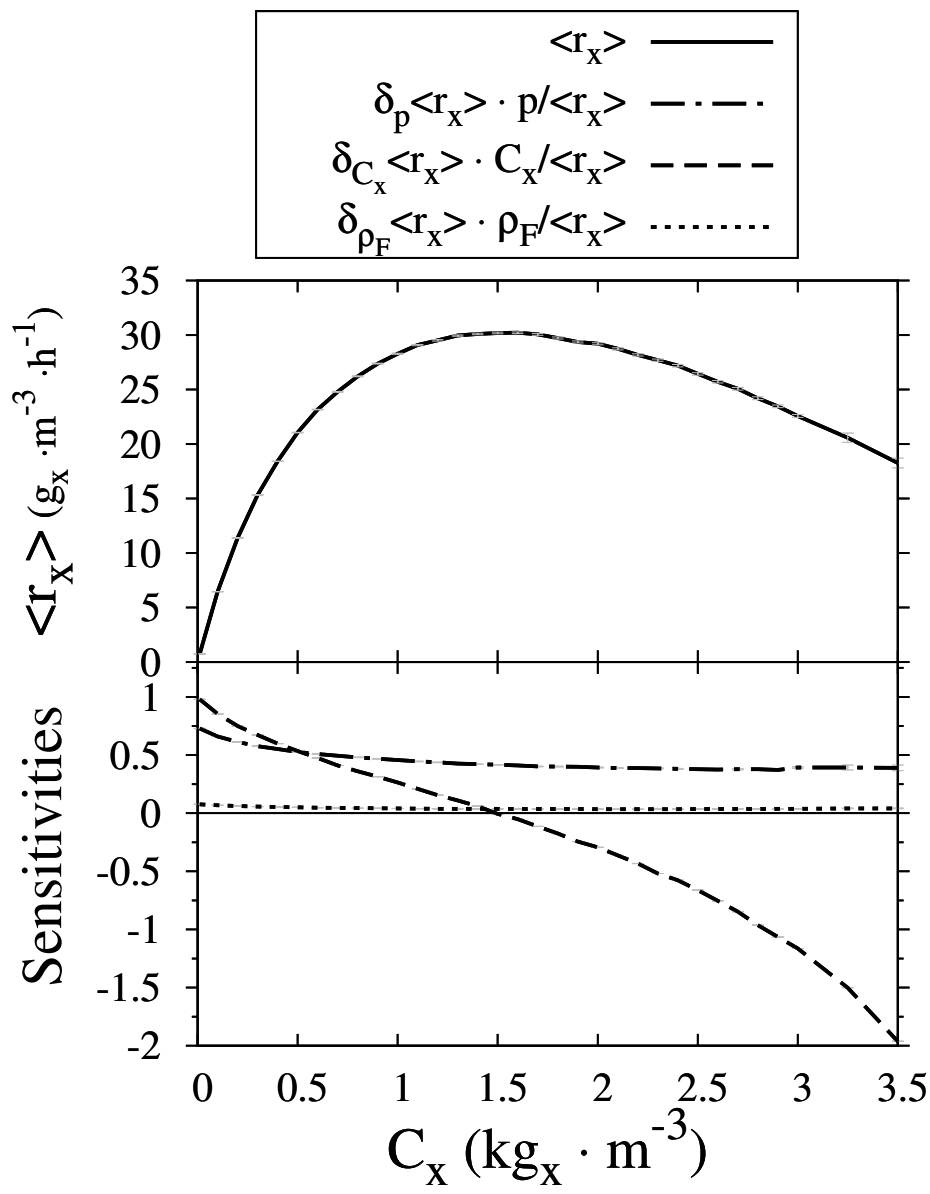

Figure 29: The volumetric biomass growth rate $\left\langle R_{x}>\right.$ and its sensitivity parameters $\partial_{p}\left\langle r_{x}\right\rangle, \partial_{C_{x}}\left\langle r_{x}\right\rangle$, and $\partial_{\rho_{\mathcal{F}}}\left\langle r_{x}\right\rangle$ in a $25 \mathrm{~L}$ DiCoFluV photobioreactor (see Fig. 26) operating in continuous mode and cultivating Chlamydomonas reinhardtii. The results were obtained with the algorithm presented in Section 4.3 for $10^{6}$ realizations, as a function of the dry-biomass concentration $C_{x}$. Statistical estimation of the numerical error is provided as error bars (gray). Relative types of sensitivity are shown, i.e., $\partial_{\pi}\left\langle r_{x}\right\rangle \times \frac{\pi}{\left\langle r_{x}\right\rangle}$, where $\partial_{\pi}$ is the partial derivative with respect to the parameter $\pi$ under study: $p$ is pigment content, $C_{x}$ is the dry-biomass concentration, and $\rho^{\mathcal{F}}$ is reflectivity of the optical-fiber surface (see Fig. 26).

Fig. 29 shows the results obtained from simulations of the whole predictive model (from calculations of optical and radiative properties to determination of the growth rate) presented in this chapter for $C$. reinhardtii cultivated in a geometrically complex photobioreactor with a thousand internal optical fibers (see [7, 80] and Fig. 26). At each scale, the model was solved by the integral Monte Carlo method, including the volume integral, which implies formulation of the non-linear coupling law Eq. 163 (see Section 4.3). 
The CPU time ranged from 1 to 6 min for typical biomass concentrations varying between 0.5 and $3 \mathrm{~g} / \mathrm{L}$ $\left(2 \times 10^{5}\right.$ realizations, standard deviation $1 \%$, on Intel Core i7-2720QM). The DiCoFluV photobioreactor, with its complex geometric structure, obeys the same engineering rule as the rules classically developed for one-dimensional geometric structure $[2,6,7]$ : we identified an optimal biomass concentration that yields maximal productivity. Using the method presented in Section 4.4, we estimated the sensitivity of $\left\langle r_{x}\right\rangle$ to different parameters (biomass concentration $C_{x}$, optical-fiber reflectivity $\rho^{\mathcal{F}}$, and pigment content $p$ ) simultaneously with $\left\langle r_{x}\right\rangle$. The sensitivity to $C_{x}$ is simply the slope of the curve $\left\langle r_{x}\right\rangle$ versus $C_{x}$ in the upper part of the figure. For optimal operation, when $\partial_{C_{x}}\left\langle r_{x}\right\rangle$ is positive, the biomass concentration should be increased, but when $\partial_{C_{x}}\left\langle r_{x}\right\rangle$ is negative, $C_{x}$ should be decreased. Of course, the optimum corresponds to $\partial_{C_{x}}\left\langle r_{x}\right\rangle=0$. Estimation of $\left.\partial_{C_{x}}<r_{x}\right\rangle$ is especially relevant for maintenance of optimal operation without evaluating $\left\langle r_{X}\right\rangle$ for the full range of biomass concentration. The sensitivity to $\rho^{\mathcal{F}}$ is low but positive, indicating that the fiber reflectivity should be increased to reduce photon losses at the boundary. Finally, $\partial_{p}\left\langle r_{x}\right\rangle$ indicates that diminution of pigment content in microbial cells leads to homogenization of the radiation field, but the overall energy absorbed by the culture decreases, and losses at the fibers' and the photobioreactor's surface increase. This sensitivity is positive; therefore, the pigment content should be increased to improve performance of the photobioreactor.

\section{Acknowledgments}

This work has been sponsored by the French government's research program "Investissements d'avenir" through the ANR programs PHOTOBIOH2 (2005-08), BIOSOLIS (2008-11), ALGOH2 (2011-15), PRIAM (2013-15), and the IMobS3 Laboratory of Excellence (ANR-10-LABX-16-01); by the European Union through the program "Regional competitiveness and employment" 2007-2013 (ERDF Auvergne region); and by the Auvergne region. This work was also funded by the CNRS through the PIE program PHOTORAD (2010-11) and the PEPS program "Intensification des transferts radiatifs pour le developpement de photobioreacteurs a haute productivite volumique" (2012-13). The authors wish to acknowledge the ESA/ESTEC for financial support through the MELiSSA project.

\section{References}

[1] J. R. Bolton, D. O. Hall, The maximum efficiency of photosynthesis, Photochemistry and Photobiology 53 (4) (1991) $545-548$.

[2] J. Pruvost, J.-F. Cornet, Knowledge models for the engineering and optimization of photobioreactors, Chap. 10 in Microalgal Biotechnology vol. 1, Potential Production, De Gruyter GmbH \& Co. KG, 2012.

[3] S. Aiba, Growth kinetics of photosynthetic micro-organisms, Adv. Biochem. Eng. 23 (1982) 85-156.

[4] J. F. Cornet, C. G. Dussap, G. Dubertret, A structured model for simulation of cultures of the cyanobacterium spirulina platensis in photobioreactors: I. coupling between light transfer and growth kinetics, Biotechnology and Bioengineering 40 (7) (1992) 817-825.

[5] J.-F. Cornet, C. Dussap, J.-B. Gros, Kinetics and energetics of photosynthetic micro-organisms in photobioreactors, in: Bioprocess and Algae Reactor Technology, Apoptosis, Vol. 59 of Advances in Biochemical Engineering Biotechnology, Springer Berlin Heidelberg, 1998, pp. 153-224.

[6] J. F. Cornet, C. G. Dussap, A simple and reliable formula for assessment of maximum volumetric productivities in photobioreactors, Biotechnology Progress 25 (2) (2009) 424-435.

[7] J. F. Cornet, Calculation of optimal design and ideal productivities of volumetrically lightened photobioreactors using the constructal approach, Chemical Engineering Science 65 (2) (2010) 985-998.

[8] H. Takache, G. Christophe, J.-F. Cornet, J. Pruvost, Experimental and theoretical assessment of maximum productivities for the microalgae chlamydomonas reinhardtii in two different geometries of photobioreactors, Biotechnology Progress 26 (2) (2010) 431-440.

[9] L. Pilon, H. Berberoglu, R. Kandilian, Radiation transfer in photobiological carbon dioxide fixation and fuel production by microalgae, Journal of Quantitative Spectroscopy and Radiative Transfer 112 (17) (2011) 2639-2660.

[10] Z. Csogor, M. Herrenbauer, K. Schmidt, C. Posten, Light distribution in a novel photobioreactor - modelling for optimization, Journal of Applied Phycology 13 (2001) 325-333.

[11] B. Farges, C. Laroche, J. F. Cornet, C. G. Dussap, Spectral kinetic modeling and long-term behavior assessment of arthrospira platensis growth in photobioreactor under red (620 nm) light illumination, Biotechnology Progress 25 (1) (2009) 151-162.

[12] I. Prigogine, Introduction to thermodynamics of irreversible processes, New York: Interscience, $1967,3 \mathrm{rd}$ ed. 1. 
[13] J. W. Stucki, Stability analysis of biochemical systems - a practical guide, Progress in Biophysics and Molecular Biology 33 (1979) $99-187$.

[14] C.-G. Dussap, Etude thermodynamique et cinetique de la production de polysaccharides microbiens par fermentation en limitation par le transfert d'oxygene, Thèse d'Etat $n^{\circ}$ d'ordre 409, Université Blaise Pascal de Clermont Ferrand, 1988.

[15] H. Berberoglu, L. Pilon, A. Melis, Radiation characteristics of chlamydomonas reinhardtii cc125 and its truncated chlorophyll antenna transformants tla1, tlax and tla1-cw+, International Journal of Hydrogen Energy 33 (22) (2008) 6467-6483.

[16] H. Berberoglu, P. S. Gomez, L. Pilon, Radiation characteristics of botryococcus braunii, chlorococcum littorale, and chlorella sp. used for CO2 fixation and biofuel production, Journal of Quantitative Spectroscopy and Radiative Transfer 110 (17) (2009) 1879-1893.

[17] M. Chami, A. Thirouard, T. Harmel, Polvsm (polarized volume scattering meter) instrument: an innovative device to measure the directional and polarized scattering properties of hydrosols, Optics express 22 (21) (2014) 26403.

[18] M. P. Mengüç, Characterization of fine particles via elliptically-polarized light scattering, Purdue Heat Transfer Celebration (2002)

[19] O. Muoz, J. W. Hovenier, Laboratory measurements of single light scattering by ensembles of randomly oriented small irregular particles in air. a review, Journal of Quantitative Spectroscopy and Radiative Transfer 112 (11) (2011) 16461657.

[20] M. J. Berg, N. R. Subedi, P. A. Anderson, N. B. Fowler, Using holography to measure extinction, Optics letters 39 (13) (2014) 3993.

[21] M. I. Mishchenko, M. J. Berg, C. M. Sorensen, C. V. van der Mee, On definition and measurement of extinction cross section, Journal of Quantitative Spectroscopy and Radiative Transfer 110 (2009) 323-327.

[22] R. Vaillon, J. M. Geffrin, C. Eyraud, O. Merchiers, P. Sabouroux, B. Lacroix, A novel implementation of a microwave analog to light scattering measurement set-up, Journal of Quantitative Spectroscopy and Radiative Transfer 112 (11) (2011) 1753-1760.

[23] T. Wriedt, Light scattering theories and computer codes, Journal of Quantitative Spectroscopy and Radiative Transfer 110 (11) (2009) 833-843.

[24] F. M. Kahnert, Numerical methods in electromagnetic scattering theory, Journal of Quantitative Spectroscopy and Radiative Transfer 7980 (0) (2003) 775-824.

[25] J. Dauchet, S. Blanco, J.-F. Cornet, R. Fournier, Calculation of the radiative properties of photosynthetic microorganisms, Journal of Quantitative Spectroscopy and Radiative Transfer 161 (2015) 60-84.

[26] J. F. Cornet, C. G. Dussap, P. Cluzel, G. Dubertret, A structured model for simulation of cultures of the cyanobacterium Spirulina platensis in photobioreactors: II. identification of kinetic parameters under light and mineral limitations, Biotechnology and Bioengineering 40 (7) (1992) 826-834.

[27] L. Pottier, J. Pruvost, J. Deremetz, J. F. Cornet, J. Legrand, C. G. Dussap, A fully predictive model for one-dimensional light attenuation by chlamydomonas reinhardtii in a torus photobioreactor, Biotechnol. Bioeng. 91 (5) (2005) 569-582.

[28] I. Thormählen, J. Straub, U. Grigull, Refractive index of water and its dependence on wavelength, temperature, and density, Journal of Physical and Chemical Reference Data 14 (1985) 933-945.

[29] M. Mishchenko, L. Travis, A. Lacis, Scattering, absorption, and emission of light by small particles, Cambridge Univ Pr, 2002, a Lorenz-Mie code is available at www.giss.nasa.gov/staff/mmishchenko.

[30] S. Bernard, T. A. Probyn, A. Quirantes, Simulating the optical properties of phytoplankton cells using a two-layered spherical geometry, Biogeosciences Discussions 6 (1) (2009) 1497-1563.

[31] T. T. Wu, J. Y. Qu, M. Xu, Unified Mie and fractal scattering by biological cells and subcellular structures, Optics Letters 32 (16) (2007) 2324-2326.

[32] W. Choi, C.-C. Yu, C. Fang-Yen, K. Badizadegan, R. Dasari, M. S. Feld, Field-based angle-resolved light-scattering study of single live cells, Optics Letters 33 (14) (2008) 1596-1598.

[33] L. Kolokolova, B. A. S. Gustafsonm, Scattering by inhomogeneous particles: microwave analog experiments and comparison to effective medium theories, Journal of Quantitative Spectroscopy and Radiative Transfer 70 (4-6) (2001) 611-625.

[34] H. C. van de Hulst, Light scattering by small particules, Dover Publication, Inc., 1981.

[35] C. F. Bohren, D. R. Huffman, Absorption and scattering of light by small particles, New York, Wiley-Interscience, 1983.

[36] M. I. Mishchenko, J. W. Hovenier, L. D. Travis, Light scattering by nonspherical particles: theory, measurements, and applications, Academic Press, 2000.

[37] T. Nousiainen, Optical modeling of mineral dust particles: A review, Journal of Quantitative Spectroscopy and Radiative Transfer 110 (14-16) (2009) 1261-1279.

[38] A. J. Baran, A review of the light scattering properties of cirrus, Journal of Quantitative Spectroscopy and Radiative Transfer 110 (14-16) (2009) 1239-1260.

[39] L. Bi, P. Yang, Impact of calcification state on the inherent optical properties of emiliania huxleyi coccoliths and coccolithophores, Journal of Quantitative Spectroscopy and Radiative Transfer 155 (2015) 10-21.

[40] L. Bi, P. Yang, Modeling of light scattering by biconcave and deformed red blood cells with the invariant imbedding t-matrix method, Journal of biomedical optics 18 (5) (2013) 055001-055001.

[41] A. E. Moskalensky, M. A. Yurkin, A. I. Konokhova, D. I. Strokotov, V. M. Nekrasov, A. V. Chernyshev, G. A. Tsvetovskaya, E. D. Chikova, V. P. Maltsev, Accurate measurement of volume and shape of resting and activated blood platelets from light scattering, Journal of biomedical optics 18 (1) (2013) 017001-017001.

[42] M. A. Yurkin, A. G. Hoekstra, The discrete-dipole-approximation code adda: capabilities and known limitations, Journal of Quantitative Spectroscopy and Radiative Transfer 112 (13) (2011) 2234-2247.

[43] J. Hellmers, V. Schmidt, T. Wriedt, Improving the numerical stability of t-matrix light scattering calculations for extreme 
particle shapes using the nullfield method with discrete sources, Journal of Quantitative Spectroscopy and Radiative Transfer 112 (11) (2011) 1679-1686.

[44] P. J. Wyatt, Light scattering in the microbial world, Journal of Colloid and Interface Science 39 (3) (1972) $479-491$.

[45] A. Morel, A. Bricaud, Theoretical results concerning light absorption in a discrete medium, and application to specific absorption of phytoplankton, Deep Sea Research Part A. Oceanographic Research Papers 28 (11) (1981) $1375-1393$.

[46] A. Bricaud, A. Morel, Light attenuation and scattering by phytoplanktonic cells: a theoretical modeling, Appl. Opt. 25 (4) (1986) 571-580.

[47] S. Bernard, T. A. Probyn, R. G. Barlow, Measured and modelled optical properties of particulate matter in the southern benguela, South African Journal of Science 97 (9-10) (2001) 410-420.

[48] P. Yang, Z. Zhang, B. A. Baum, H.-L. Huang, Y. Hu, A new look at anomalous diffraction theory (adt): Algorithm in cumulative projected-area distribution domain and modified adt, Journal of Quantitative Spectroscopy and Radiative Transfer 89 (1-4) (2004) 421-442.

[49] L. Liu, M. I. Mishchenko, W. P. Arnott, A study of radiative properties of fractal soot aggregates using the superposition t-matrix method, Journal of Quantitative Spectroscopy and Radiative Transfer 109 (15) (2008) 2656 - 2663.

[50] L. I. Schiff, Approximation method for high-energy potential scattering, Physical Review 103 (2) (1956) $443-453$.

[51] J. Charon, S. Blanco, J.-F. Cornet, J. Dauchet, M. El Hafi, R. Fournier, M. Kaisar Abboud, S. Weitz, Monte Carlo implementation of schiffs approximation for estimating radiative properties of homogeneous, simple-shaped and optically soft particles: Application to photosynthetic micro-organisms, Journal of Quantitative Spectroscopy and Radiative Transfer In press.

[52] A. H. Sihvola, Electromagnetic mixing formulas and applications, IEE, London, 1999.

[53] N. Hoepffner, S. Sathyendranath, Effect of pigment composition on absorption properties of phytoplankton, Marine Ecology Progress Series 73 (1) (1991) 11-23.

[54] R. R. Bidigare, M. E. Ondrusek, J. H. Morrow, D. A. Kiefer, In-vivo absorption properties of algal pigments, Vol. 1302, SPIE, 1990, pp. 290-302.

[55] B. Wozniak, J. Dera, D. Ficek, R. Majchrowski, S. Kaczmarek, M. Ostrowska, O. I. Koblentz-Mishke, Model of the in vivo spectral absorption of algal pigments. part 1. Mathematical apparatus, Oceanologia 42 (2) (2000) 177-190.

[56] StarWest, Edstar development environment project, http://edstar.lmd.jussieu.fr/databases.

[57] K. R. Naqvi, M. N. Merzlyak, T. B. Melo, Absorption and scattering of light by suspensions of cells and subcellular particles: an analysis in terms of kramers-kronig relations, Photochem. Photobiol. Sci. 3 (2004) $132-137$.

[58] P. S. Tuminello, E. T. Arakawa, B. N. Khare, J. M. Wrobel, M. R. Querry, M. E. Milham, Optical properties of bacillus subtilis spores from 0.2 to $2.5 \nu \mathrm{m}$, Applied Optics 36 (13) (1997) 2818-2824.

[59] V. Lucarini, Kramers-Kronig relations in optical materials research, Springer, 2005.

[60] E. Aas, Refractive index of phytoplankton derived from its metabolite composition, Journal of Plankton Research 18 (12) (1996) 2223-2249.

[61] R. Siegel, J. R. Howell, Thermal radiation heat transfer, Hemisphere Pub. Corp., 1981.

[62] K. M. Case, P. F. Zweifel, Linear Transport Theory, Addison-Wesley, Reading, Mass, 1967.

[63] R. M. Goody, Y. L. Yung, Atmospheric radiation: theoretical basis, Clarendon Press, 1964.

[64] S. Blanco, R. Fournier, An invariance property of diffusive random walks, EPL (Europhysics Letters) 61 (2) (2003) 168.

[65] S. Blanco, R. Fournier, Short-path statistics and the diffusion approximation, Physical Review Letters 97 (23) (2006) 230604.

[66] A. Ishimaru, Wave Propagation and Scattering in Random Media, John Wiley \& Sons, 1999.

[67] R. E. Marshak, The Milne problem for a large plane slab with constant source and anisotropic scattering, Phys. Rev. 72 (1947) 47-50.

[68] D. J. Durian, Influence of boundary reflection and refraction on diffusive photon transport, Phys. Rev. E 50 (1994) $857-866$.

[69] J. Dauchet, Analyse radiative des photobioréacteurs, Ph.D. dissertation, Université Blaise Pascal de Clermont Ferrand, $n^{\circ} 2304,2012$.

[70] J. F. Cornet, C. G. Dussap, J. B. Gros, Conversion of radiant light energy in photobioreactors, AIChE Journal 40 (6) (1994) 1055-1066.

[71] J. F. Cornet, C. G. Dussap, J. B. Gros, C. Binois, C. Lasseur, A simplified monodimensional approach for modeling coupling between radiant light transfer and growth kinetics in photobioreactors, Chemical Engineering Science 50 (9) (1995) 1489-1500.

[72] H. Takache, J. Pruvost, J. F. Cornet, Kinetic modeling of the photosynthetic growth of chlamydomonas reinhardtii in a photobioreactor, Biotechnology Progress 28 (3) (2012) 681-692.

[73] A. Schuster, Radiation through a foggy atmosphere, Astrophysical Journal 21 (1905) 1.

[74] H. C. Hottel, A. F. Sarofim, Radiative transfer, 1967.

[75] M. Meng, R. Viskanta, Comparison of radiative transfer approximations for a highly forward scattering planar medium, Journal of Quantitative Spectroscopy and Radiative Transfer 29 (5) (1983) 381 - 394.

[76] N. Metropolis, S. Ulam, The Monte Carlo method, Journal of the American statistical association 44 (247) (1949) $335-341$.

[77] J. M. Hammersley, D. C. Handscomb, Monte Carlo Methods, Chapman and Hall, London, 1964.

[78] J. R. Howell, The Monte Carlo method in radiative heat transfer, Journal of Heat Transfer 120 (3) (1998) 547-560.

[79] J. Delatorre, G. Baud, J. Bézian, S. Blanco, C. Caliot, J. Cornet, C. Coustet, J. Dauchet, M. El Hafi, V. Eymet, R. Fournier, J. Gautrais, O. Gourmel, D. Joseph, N. Meilhac, a. Pajot, M. Paulin, P. Perez, B. Piaud, M. Roger, J. Rolland, F. Veynandt, S. Weitz, Monte Carlo advances and concentrated solar applications, Solar Energy 103 (2014) 
$653-681$.

[80] J. Dauchet, S. Blanco, J.-F. Cornet, M. El Hafi, V. Eymet, R. Fournier, The practice of recent radiative transfer Monte Carlo advances and its contribution to the field of microorganisms cultivation in photobioreactors, Journal of Quantitative Spectroscopy and Radiative Transfer 128 (2013) 52-59.

[81] M. Pharr, G. Humphreys, Physically based rendering : from theory to implementation, Morgan Kaufmann Pub. / Elsevier, 2010.

[82] V. Rochatte, J. Dauchet, J.-F. Cornet, Experimental validation and modelling of a photobioreactor operating with diluted and controlled light flux, to be published in Journal of Physics: Conference Series, Proceedings of Eurotherm Conference 105: Computational Thermal Radiation in Participating Media V, France.

[83] I. Dimov, Monte Carlo Methods for Applied Scientists, World Scientific, New Jersey, 2008.

[84] M. Kalos, P. Whitlock, Monte Carlo Methods, second ed., WileyVCH, Weinheim, 2008.

[85] M. Roger, S. Blanco, M. El Hafi, R. Fournier, Monte Carlo estimates of domain-deformation sensitivities, Physical Review Letters 95 (18) (2005) 180601

[86] H. A. Irazoqui, J. Cerdá, A. E. Cassano, The radiation field for the point and line source approximations and the threedimensional source models: Applications to photoreactions, The Chemical Engineering Journal 11 (1) (1976) $27-37$.

[87] G. Spadoni, E. Bandini, F. Santarelli, Scattering effects in photosensitized reactions, Chemical Engineering Science 33 (4) (1978) 517-524.

[88] A. E. Cassano, C. A. Martin, R. J. Brandi, O. M. Alfano, Photoreactor analysis and design : fundamentals and applications, Ind. Eng. Chem. Res. 34 (1995) 2155-2201.

[89] L. G. De La Vara, C. Gomez-Lojero, Participation of plastoquinone, cytochrome c 553 and ferrodoxin-nadp+ oxidoreductase in both photosynthesis and respiration in spirulina maxima, Photosynthesis research 8 (1) (1986) 65-78.

[90] J.-F. Cornet, L. Favier, C.-G. Dussap, Modeling stability of photoheterotrophic continuous cultures in photobioreactors, Biotechnology progress 19 (4) (2003) 1216-1227.

[91] R. F. Mouhim, J.-F. Cornet, T. Fontane, B. Fournet, G. Dubertret, Production, isolation and preliminary characterization of the exopolysaccharide of the cyanobacterium spirulina platensis, Biotechnology letters 15 (6) (1993) 567-572.

[92] J. A. Roels, Kinetics and energetics in biotechnology, Elsevier Biomedical Press, Amsterdam, 1983.

[93] G. Meszéna, H. V. Westerhoff, Non-equilibrium thermodynamics of light absorption, Journal of Physics A: Mathematical and General 32 (2) (1999) 301.

[94] G. Paillotin, Etude théorique des modes de création, de transport et d'utilisation de l'nergie d'excitation electronique chez les plantes superieures, Ph.D. dissertation, Université de Paris-Sud, centre d'Orsay, 1974.

[95] A. Bejan, Unification of three different theories concerning the ideal conversion of enclosed radiation, Trans ASME, J. Solar Energy Engineering 109 (1987) 46-51.

[96] A. Bejan, Advanced engineering thermodynamics, 1997, John Wiley and Sons Inc., New York, 1988.

[97] L. N. M. Duysens, Brookhaven symp. in biol., 1959, pp. 10-25.

[98] P. Glansdorff, I. Prigogine, R. N. Hill, Thermodynamic theory of structure, stability and fluctuations, American Journal of Physics 41 (1) (1973) 147-148.

[99] J. W. Stucki, The optimal efficiency and the economic degrees of coupling of oxidative phosphorylation, European Journal of Biochemistry 109 (1) (1980) 269-283.

[100] J. W. Stucki, Thermodynamics of energy conversion in the cells. In: From chemical to biological organization, Springer Verlag, 1988.

[101] C. B. Ould Moulaye, Calcul des propriétés de formation en solution aqueuse des composés impliqués dans les procédés biologiques et alimentaires. Prédiction et réconciliation de données; modélisation des équilibres chimiques et entre phases., Ph.D. dissertation, Université Blaise Pascal de Clermont Ferrand, num. 1033.

[102] G. Cogne, B. Lehmann, C.-G. Dussap, J.-B. Gros, Uptake of macrominerals and trace elements by the cyanobacterium Spirulina platensis (Arthrospira platensis PCC 8005) under photoautotrophic conditions: culture medium optimization, Biotechnology and bioengineering 81 (5) (2003) 588-593.

[103] P. Joliot, Oxygen evolution in algae illuminated by modulated light., in: Brookhaven symposia in biology, Vol. 19, 1966, p. 418. 